Chin. J. Astron. Astrophys. Vol. 0, No. 0, (200x) 000-000

(http://www.chjaa.org)

Chinese Journal of

Astronomy and

Astrophysics

\title{
Gamma-Ray Bursts in the Swift Era
}

\author{
Bing Zhang * \\ Department of Physics \& Astronomy, University of Nevada, Las Vegas, NV 89154-4002, USA \\ Received 2007 xxx; accepted 2007 xxx
}

\begin{abstract}
Since the successful launch of NASA's dedicated gamma-ray burst (GRB) mission, Swift, the study of cosmological GRBs has entered a new era. Here I review the rapid observational and theoretical progress in this dynamical research field during the first two-year of the Swift mission, focusing on how observational breakthroughs have revolutionized our understanding of the physical origins of GRBs. Besides summarizing how Swift helps to solve some pre-Swift mysteries, I also list some outstanding problems raised by the Swift observations. An outlook of GRB science in the future, especially in the GLAST era, is briefly discussed.
\end{abstract}

Key words: gamma-rays: bursts

\section{INTRODUCTION}

Gamma-ray bursts (GRBs) are fascinating celestial objects. These short, energetic bursts of gamma-rays mark the most violent, cataclysmic explosions in the universe, likely associated with the births of stellar-size black holes or rapidly spinning, highly magnetized neutron stars. Since the detections of their long-wavelength afterglows (Costa et al. 1997; van Paradijs et al. 1997; Frail et al. 1997), GRBs are observationally accessible in essentially all electromagnetic wavelengths. They are also potential emission sources of ultra-high energy cosmic rays, high-energy neutrinos, and gravitational waves. As stellar scale events located at cosmological distances, GRBs open a unique window to connect together the branches of stellar, interstellar, galactic, and intergalactic astronomy as well as cosmology. The study of GRBs has been prolific over the past several years. New discoveries on GRBs have been ranked several times as one of the "top-ten scientific breakthroughs of the year" by Science magazine (e.g. \#6 in 2003 and \#4 in 2005). The topic of GRBs has been extensively reviewed over the years (e.g. Fishman \& Meegan 1995; Piran 1999; van Paradijs et al. 2000; Mészáros 2002; Lu et al. 2004; Zhang \& Mészáros 2004; Piran 2005; Mészáros 2006).

The launch of the NASA's dedicated GRB mission, Swift (Gehrels et al. 2004), has opened a new era for GRB study. Carrying three instruments (Burst Alert Telescope [BAT], Barthelmy et al. 2005a; X-Ray Telescope [XRT], Burrows et al. 2005a; and UV-Optical Telescope [UVOT], Roming et al. 2005), Swift is a multi-wavelength observatory that can "swiftly" catch the

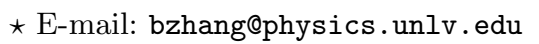


unpredictable bursts of gamma-rays in the random directions of the sky within less than 100 seconds with all three instruments on target. It allows for the first time detections of multiwavelength GRB early afterglows in a time domain previously unexplored. In slightly over two years of operation, Swift has fulfilled most of its pre-mission scientific goals in GRB study, and more importantly, brings new surprises and challenges to our understanding of these nature's most violent and mysterious explosions. The Swift revolution has been summarized in several recent reviews (e.g. Mészáros 2006; O’Brien et al. 2006a; Fox \& Mészáros 2006).

The plan of this review is the following. Since more extended reviews on Swift observational data are being written (e.g. N. Gehrels et al. 2007, in preparation), I will not invest great efforts to summarize Swift observations in a systematic manner. Rather, I will highlight the most important Swift observational results, and put more emphasis on discussing how new data revolutionize our understanding on the nature/physics of the GRB phenomenon. I do not intend to discuss GRB basics, which has been covered in an earlier review (Zhang \& Mészáros 2004), and I refer the latest full GRB review by Mészáros (2006) to those readers who are interested in both GRB basics and the latest developments in the field. The basic theme of this review is similar to Zhang \& Mészáros (2004), which includes the progress, problems and prospects in the field. However, by comparing the two reviews, it is encouraging to see that many items discussed as "problems" in the previous review are now included as part of "progress" $(\S 2-4)$. On the other hand, the list of "problems" ( 55$)$ is not shortened, mainly because new observations reveal new puzzles that were not expected before. The "prospects" part ( $\S 6)$ is as bright as before, in particular in view of the upcoming high-energy era of GRB study led by the launch of GLAST. Due to page limitation, I will make no effort to include all the important papers published in the pre-Swift era (my apologies), but will try to include most recent papers. Following an earlier ChJAA review (Cheng \& Lu 2001), I will also pay special attention to the latest contributions of the Chinese astronomers in the GRB field.

I'd like to finish the introduction with a time table of major (GRB-related) events in the first two years of operation of Swift.

- Nov. 20, 2004: the Swift satellite was successfully launched from the Cape Canaveral Air Force Base, Florida, USA;

- Dec. 27, 2004: Swift BAT detected the brightest gamma-ray events ever detected by the mankind, a giant flare from the Galactic Soft Gamma-ray Repeater source SGR 1806-20 (Palmer et al. 2005). This event was also detected by many other high energy detectors (e.g. Hurley et al. 2005; Terasawa et al. 2005). The event triggered the possibility that a good fraction of short GRBs may be simply extragalactic SGR giant flares (Hurley et al. 2005, cf. Nakar et al. 2006b);

- Jan. 26, 2005 and Feb. 19, 2005: Swift detected two bursts that show very steep decay in early X-ray afterglows (Tagliaferri et al. 2005; Goad et al. 2006). The steep decay component is later found to be norm of most early X-ray afterglows;

- Apr. 6, 2005: Swift detected its first complete X-ray flare following a soft GRB. May 2, 2005 , the second burst detected on this day by Swift showed a giant X-ray flare with fluence comparable to that of the prompt gamma-rays. The results were reported in Burrows et al. (2005b), Romano et al. (2006a), Falcone et al. (2006);

- May 9, 2005: Swift detected the first X-ray afterglow following a short duration GRB (Gehrels et al. 2005). The XRT error box overlaps with a giant elliptical galaxy in a galactic cluster at a low redshift $(z=0.225)$, giving the first evidence of the compact star merger origin of short GRBs (Gehrels et al. 2005; Bloom et al. 2006a);

- Two months later on Jul. 9, 2005, HETE-2 triggered another short GRB (Villasenor et al. 2005), leading to the discovery of the first optical afterglow of short GRBs (Fox et al. 2005; Hjorth et al. 2005); 
- Half month later on Jul. 24, 2005, another short GRB was captured by Swift, whose coordinates are firmly located inside an elliptical galaxy (but off-center) (Barthelmy et al. 2005b; Berger et al. 2005a). This conclusively suggests that short GRBs have a distinct origin from traditional long GRBs, probably associated with compact star mergers. The extended X-ray flares following GRB 050724 (Barthelmy et al. 2005b), on the other hand, pose a great challenge to the traditional compact star merger models;

- By mid 2005, a canonical XRT lightcurve emerged from the early XRT afterglow data of a sample of bursts (Nousek et al. 2006, see also Chincarini et al. 2005), which includes five distinct components (Zhang et al. 2006). Interpreting these components require new additions to the standard fireball model (Zhang et al. 2006; Nousek et al. 2006; Panaitescu et al. 2006a);

- It became clear in mid-2005 that most GRBs have very dim early optical afterglows. Most of them are not detectable by Swift UVOT (Roming et al. 2006a);

- Sep. 4, 2005: Swift detected a GRB with the highest redshift (as of the end of 2006). The detection of the burst (Cusumano et al. 2006a) prompted the IR follow-up observations which led to the identification of its redshift $z=6.29$ (Haislip et al. 2006; Kawai et al. 2006; Antonelli et al. 2005);

- Feb. 18, 2006: Swift detected an extremely long, faint, low-luminosity GRB (Campana et al. 2006a) at redshift $z=0.0331$ (Mirabal et al 2006), which is clearly associated with a Type Ic supernova SN 2006aj (Pian et al. 2006). More intriguingly, a distinct thermal X-ray emission component was detected in the XRT prompt emission spectrum, which may be related to the shock breakout of the underlying supernova (Campana et al. 2006a);

- Jun. 14, 2006: Swift detected a peculiar nearby long-duration burst (Gehrels et al. 2006), which was not associated with a supernova (Gal-Yam et al. 2006; Fynbo et al. 2006a; Della Valle et al. 2006b). This peculiar event calls for reconsideration of the GRB classification scheme;

- Oct. 7, 2006: Swift detected a very bright GRB (Schady et al. 2006a), whose early optical flux peaked around 10th magnitude, very close to the previous record-holder GRB 990123 (Alkerlof et al. 1999). The decay behavior is however rather different from GRB 990123, likely dominated by the forward shock emission (Mundell et al. 2006; Schady et al. 2006a).

\section{CLASSES OF GRBS}

One fundamental question related to GRBs is how many intrinsically different categories they have, which correspond to intrinsically different types of progenitor and possibly different types of central engine as well. This section is dedicated to this important topic.

\subsection{Short vs. long; Type I vs. Type II}

From the GRB sample collected by Burst And Transient Source Experiment (BATSE) on board the Compton Gamma-Ray Observatory (CGRO), a clear bimodal distribution of bursts was identified (Kouveliotou et al. 1993). Two criteria have been used to classify the bursts. The primary criterion is duration. A separation line of 2 seconds was adopted to separate the double-hump duration distribution of the BATSE bursts. The bimodal distribution is supported by hardness-duration correlations (Qin et al. 2000). The supplementary criterion is the hardness - usually denoted as the hardness ratio within the two energy bands of the detector. On average, short GRBs are harder, while long GRBs are softer. So the two distinct populations of bursts discussed in the literature have been long-soft GRBs and short-hard GRBs. Based on duration distribution, a third class of GRBs with intermediate duration has been proposed (e.g. Mukherjee et al. 1998; Horváth 1998; Horváth et al. 2006). The case is however not conclusive. 
Several prompt emission data analyses regarding the differences between long and short GRBs have revealed interesting conclusions. Ghirlanda et al. (2004a) discovered that the short GRBs are hard mainly because of a harder low-energy spectral index of the GRB spectral function (the Band function, Band et al. 1993). More interestingly, short GRB spectra are broadly similar to those of long GRBs if only the first 2 seconds of data of long GRBs are taken into account. Nakar \& Piran (2002) found that temporal properties of short GRBs are also similar to those of long GRBs in the first 1-2 seconds, with highly variable temporal structures. Liang et al. (2002), on the other hand, found that the variability time scales of short GRBs are much shorter than those of long GRBs. Dong \& Qin (2005) and Qin \& Dong (2005) present the arguments that the properties of short GRBs are different from the first two seconds of long GRBs. Cui et al. (2005) discovered that long and short GRBs follow two distinct sequences in the $E_{p}$ - hardness ratio sequence. Spectral lag (the lag of arrival time between softer band emission with respect to harder band emission) analyses indicate that the lag in short GRBs is much smaller than that in long GRBs (Yi et al. 2006; Norris \& Bonnel 2006; Gehrels et al. 2006), consistent with being zero. In both long and short GRBs, on the other hand, the ratios between lags and pulse widths are comparable. (Yi et al. 2006). This generally explains the much smaller lags in short GRBs since their pulses are much narrower.

Afterglow observations shed light onto the nature of these two distinct classes of bursts. Since 1997 and by Nov. 20, 2006, the afterglows of over 200 long GRBs have been detected (Greiner 2006). Several cases of solid associations between GRBs and Type Ib/c Supernovae have been established, which include GRB 980425/SN 1998bw at $z=0.0085$ (Galama et al. 1998; Kulkarni et al. 1998), GRB 030329/SN 2003dh at $z=0.168$ (Stanek et al. 2003; Hjorth et al. 2003), GRB 031203/SN 2003lw at $z=0.105$ (Malesani et al. 2004), GRB 060218/SN 2006aj at $z=0.0331$ (Modjaz et al. 2006; Pian et al. 2006; Sollerman et al. 2006; Mirabal et al. 2006; Cobb et al. 2006), and GRB 050525A/SN 2005nc at $z=0.606$ (Della Valle et al. 2006a). In some other cases, red SNe bumps have been observed in the late optical afterglow light curves (Bloom et al. 1999a, 2002; Reichart 1999; Della Valle et al. 2003; Fynbo et al. 2004; see a comprehensive sample in Zeh et al. 2004 and references therein). The host galaxies of long GRBs are exclusively star-forming galaxies, predominantly irregular dwarf galaxies (Fruchter et al. 2006). All these strongly suggest that most, if not all, long GRBs are produced during the core-collapses of massive stars, dubbed "collapsars", as has been suggested theoretically (Woosley 1993; Paczyński 1998; MacFadyen \& Woosley 1999; Colgate 1974). Not long ago, it has been suggested that both observations and theories are consistent with the hypothesis that every long GRB has an underlying supernova associated with it (Woosley \& Bloom 2006).

The observations led by Swift (and in small number by HETE-2) have revealed a completely different picture for "short" GRBs. Since the watershed discoveries of the first three short GRB afterglows (GRB 050509B at $z=0.226$, Gehrels et al. 2005, Bloom et al. 2006a; GRB 050709 at $z=0.1606$, Villasenor et al. 2005, Fox et al. 2005, Hjorth et al. 2005; and GRB 050724 at $z=0.258$, Barthelmy et al. 2005b, Berger et al. 2005a), by Nov. 20, 2006, a total number 12 "short" GRB (with duration shorter than 5 seconds, Donaghy et al. 2006, see Table 1) afterglows have been discovered. The general message collected from these observations is that they are intrinsically different from long GRBs. GRB 050509B (Gehrels et al. 2005; Bloom et al. 2006) and GRB 050724 (Barthelmy et al. 2005b; Berger et al. 2005a) are found to be at the outskirts of elliptical galaxies, in which star forming rate is very low (Fig.1). It is rather unlikely that these two events are associated with deaths of massive stars. GRB 050709 (Fox et al. 2005) and GRB 051221A (Soderberg et al. 2006b) are associated with star-forming galaxies, but they are usually far away from the star forming regions. There are several other cases for which a robust host galaxy was not identified, but the host galaxy candidates are of early type (e.g. GRB 060121 Levan et al. 2006a; GRB 060502B, Bloom et al. 2006b). Deep supernova searches 

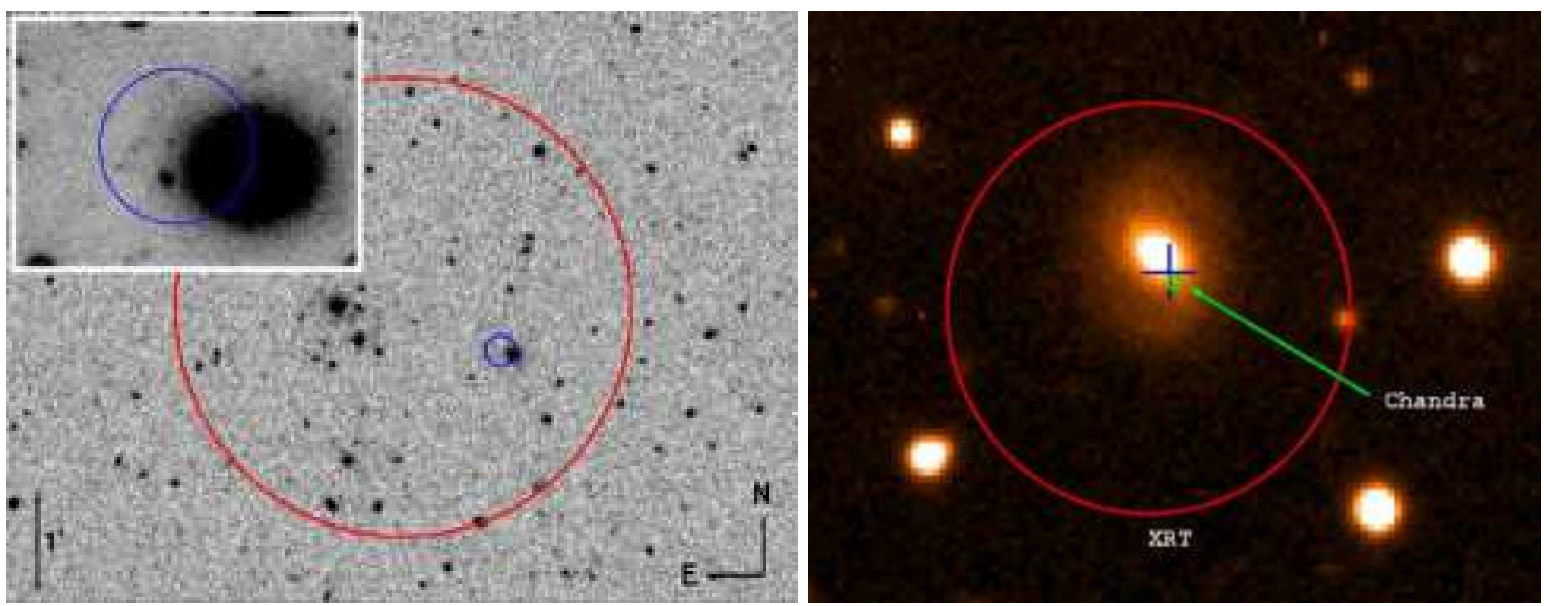

Fig. 1 Two Swift short GRBs associated with elliptical galaxies. left: GRB 050509B (Gehrels et al. 2005; Bloom et al. 2006a), the red and blue circles are BAT and XRT error boxes, respectively; Right: GRB 050724 (Barthelmy et al. 2005b; Berger et al. 2005a).

have been performed, but with negative results (e.g. for GRB 050509B, Bloom et al. 2006a; GRB 050709, Fox et al. 2005; GRB 050724, Berger et al. 2005; GRB 050813, Ferrero et al. 2006; GRB 060505, Fynbo et al. 2006). All these are consistent with the long-held speculation that some cosmological GRBs are associated with mergers of compact objects, such as neutron star - neutron star (NS-NS) mergers, neutron star - black hole (NS-BH) mergers, white dwarf - black hole (WD-BH) mergers, WD-NS mergers, and even WD-WD mergers (e.g. Paczýnski 1986; Goodman 1986; Eichler et al. 1989; Paczýnski 1991; Narayan et al. 1992; Mészáros \& Rees 1992; Ruffert \& Janka 1999; Fryer et al. 1999; Rosswog et al. 2003; Aloy et al. 2005; Dermer \& Atoyan 2006; King et al. 2007; Levan et al. 2006c). These mergers only involve evolved compact stars, and can happen in early type galaxies (such as elliptical galaxies). On the other hand, population studies reveal some novel channels to form compact star mergers in a relatively short time scale (Belczynski et al. 2002, 2006). This allows some merger events to happen in starforming galaxies. In any case, since there is a significant delay in time since the birth of the two compact stars before a coalescence happens (due to the loss of orbital angular momentum via gravitational radiation), the merger events tend to happen in the outskirts of the host galaxy since NSs usually receive a large "kick" velocity at birth (Bloom et al. 1999b, cf. Grindlay et al. 2006). Although some weak nuclear radioactivity signals would accompany the merger events (e.g. Li \& Paczyński 1998; Kulkarni 2005), they are nonetheless much fainter than the typical Type Ib/c supernovae that accompany long GRBs. All these suggest that the observations of "short" GRBs are consistent with the compact star merger scenario.

One important fact from the recent short GRB observations is that they are not necessarily short. Extended emission following short GRBs has been seen in about $1 / 3$ of the sample in Table 1 (Norris \& Bonnell 2006). GRB 050724 (and likely also GRB 050709) was followed by erratic X-ray flares that have properties similar to the prompt emission and require restart of the central engine (Barthelmy et al. 2005b; Zhang et al. 2006). There has been evidence of extended emission following short GRBs in the pre-Swift era (e.g. Lazzati et al. 2001; Connaughton 2002). A closer investigation reveals that a larger (than 1/3) fraction of BATSE short GRBs actually harbor observable extended emission (Norris \& Gehrels 2007). All these greatly challenge the standard merger paradigm. Donaghy et al. (2006) suggested to increase the separation line between short and long GRBs to 5 seconds. 
Table 1 Durations, redshifts, host galaxies of Type I ("short"-hard) GRBs with afterglow detections before Nov. 20, 2006. Bursts marked with '*' have durations longer than 5 seconds. Several other short GRBs without afterglow detections include GRBs 050906, 050925, 051105A, 051114, and 051211A, which are not listed in the table.

\begin{tabular}{lcccccc}
\hline GRB & Mission & $T_{90}(\mathrm{~s})$ & $z$ & Host galaxy & Location & Refs \\
\hline $050509 B$ & Swift & $0.04 \pm 0.004$ & 0.226 & elliptical & outskirts? & {$[1,2]$} \\
050709 & HETE & $0.07 \pm 0.01$ & 0.1606 & irregular & outskirts & {$[3-5]$} \\
050724 & Swift & $3.0 \pm 1.0$ & 0.257 & elliptical & outskirts & {$[6-9]$} \\
050813 & Swift & $0.6 \pm 0.1$ & - & - & - & {$[10]$} \\
$050911^{*}$ & Swift & $\sim 16$ & $0.1646 ?$ & galaxy cluster? & - & {$[11,12]$} \\
051210 & Swift & $1.4 \pm 0.2$ & - & - & - & {$[13]$} \\
$051221 \mathrm{~A}$ & Swift & $1.4 \pm 0.2$ & 0.5465 & star forming galaxy & slightly off-center & {$[14,15]$} \\
$051227 *$ & Swift & $8.0 \pm 0.2$ & - & - & - & {$[16,17]$} \\
060121 & HETE & $4.25 \pm 0.56$ & $1.7 ?$ or $4.6 ?$ & early-type? & outskirts? & {$[18-20]$} \\
060313 & Swift & $0.7 \pm 0.1$ & - & - & - & {$[21]$} \\
$060502 B$ & Swift & $0.09 \pm 0.02$ & $0.287 ?$ & early-type? & outskirts? & {$[22,23]$} \\
060505 & Swift & $4.0 \pm 1.0$ & $0.089 ?$ & star-forming galaxy & - & {$[24-26]$} \\
$060614^{*}$ & Swift & $102 \pm 5$ & 0.125 & star-forming galaxy & off-center & {$[27,28]$} \\
060801 & Swift & $\sim 0.50$ & $1.1304 ? ?$ & - & - & {$[29,30]$} \\
061006 & Swift & $\sim 0.42$ & - & - & - & {$[31,30]$} \\
\hline
\end{tabular}

References: [1] Gehrels et al. (2005); [2] Bloom et al. (2006a); [3] Villasenor et al. (2005); [4] Fox et al. (2005); [5] Hjorth et al. (2005); [6] Barthelmy et al. (2005b); [7] Berger et al. (2005); [8] Campana et al. (2006b); [9] Grupe et al. 2006a; [10] Retter et al. (2005); [11] Page et al. (2006a); [12] Berger et al. (2006a); [13] La Parola et al. (2006); [14] Soderberg et al. (2006b); [15] Burrows et al. (2006); [16] Barbier et al. (2006); [17] Barthelmy et al. (2006); [18] Donaghy et al. (2006); [19] de Ugarte Postigo et al. (2006); [20] Levan et al. (2006a); [21] Roming et al. (2006b); [22] Troja et al. (2006); [23] Bloom et al. (2006b); [24] Palmer et al. (2006); [25] Ofek et al. (2006); [26] Fynbo et al. (2006); [27] Gehrels et al. (2006); [28] Mangano et al. (2007b); [29] Racusin et al. (2006); [30] Berger et al. (2006b); [31] Krimm et al. (2006a).

The discovery of GRB 060614 at $z=0.125$ (Gehrels et al. 2006; Mangano et al. 2007b) pushes this issue to the extreme, and breaks the clean dichotomy of the long vs. short classification regime ${ }^{1}$. With a duration of $\sim 100 \mathrm{~s}$ (which securely places it to the "long" category), deep searches of an underlying supernova associated with this burst came up empty-handed - any underlying supernova is more than 100 times fainter than other SNe associated with long GRBs, and is fainter than any SN ever observed (Gal-Yam et al. 2006; Fynbo et al. 2006a; Della Valle et al. 2006b). More intriguingly, the spectral lag of the burst is very short - consistent with being a short GRB (Gehrels et al. 2006). The host galaxy has a relatively low star forming rate with respect to other hosts of long GRBs (Gal-Yam et al. 2006; Della Valle et al. 2006b; Fynbo et al. 2006a), and the afterglow is located in a region far away from the center of the star forming region (Gal-Yam et al. 2006). These aspects seem to be consistent with the properties of short GRBs. Although the duration is long, a closer look at the lightcurve reveals early hard spikes (about 5 seconds) followed by a softer emission tail with spectrum rapidly softening with time (Gehrels et al. 2006; Zhang et al. 2007a; Mangano et al. 2007b). More interestingly, the total

1 There are concerns about whether the association of GRB 060614 with the nearby host galaxy is due to a chance coincidence, e.g. Schaefer \& Xiao 2006; Cobb et al. 2006b. However, Swift UVOT observation of the burst sets an upper limit to the burst redshift to be lower than 1, Gehrels et al. 2006, which rules out the higher redshifts suggested by those authors. 
energy of GRB 060614 is about 8 times of that of GRB 050724, the only "short" GRB that is robustly associated with an elliptical galaxy. Assuming an empirical relation between isotropic energy (or luminosity) and spectral peak energy (the so-called Amati-relation, $E_{p} \propto E_{\text {iso }}^{1 / 2}$, see $\S 3.4$ for more discussion), which is found generally valid among bursts (Amati et al. 2002; Amati 2006) and also within a same burst (Liang et al. 2004), Zhang et al. (2007a) generated a pseudoburst that is about 8 times less energetic. They found that this synthetic burst is "short" with $T_{90} \sim 4.4 \mathrm{~s}$ in the BATSE band. The late soft gamma-ray tails are shifted to the X-ray band as X-ray flares. This is essentially a carbon-copy of GRB 050724. This suggests that GRB 060614 is likely simply a more energetic version of GRB 050724, and should belong to merger-type GRBs (Fig.2). Another point is that the beaming-corrected gamma-ray energy of GRB 060614 is comparable to those of other short GRBs, but is about an order of magnitude less than long ones (Mangano et al. 2007). Although the possibility that GRB 060614 stands for a third type of GRBs is not ruled out (e.g. collapsars without supernova signature, Woolsey 1993), it appears that GRB 060614 is a close relative of GRB 050724. It is worth commenting that some BATSE bursts (e.g. trigger 2703) has the similar properties as GRB 060614 (e.g. Norris $\&$ Bonneli 2006). These bursts have a larger intensity ratio between the extended emission and the prompt emission than most other short GRBs (Norris \& Gehrels 2007).

With such a connection, the traditional "short vs. long" classification regime breaks down, and some new terminologies involving multiple criteria are needed to define the two GRB categories. Zhang et al. (2007a) suggest the terms "Type I' and "Type II", by analogy with the supernovae nomenclature (e.g. Filippenko 1997). As summarized in Zhang et al. (2007a) and Zhang (2006), Type I (the previous short-hard) GRBs are usually short (but may have long soft tails) and hard (but the tail may be soft), with very short spectral lags and with no supernova associations. Like Type Ia supernova, Type I GRBs are associated with old stellar population and can be found in all types of host galaxies including elliptical galaxies, and are typically in regions with low star forming rate, which is usually outskirts of the host galaxy. The most likely progenitor candidates are compact star mergers, which involve binary systems, again similar to Type Ia SNe. On the other hand, Type II (the previous long-soft) GRBs are usually long and soft, with long spectral lags and supernova associations. Like Type II supernovae, they involve core collapses of massive stars, which belong to the young stellar population. Their host galaxies are late type, predominantly irregular, dwarf galaxies. The location is usually near the center of the star-forming core of the host galaxy. According to this new definition, GRB 060614 belongs to Type I. In fact, two other Swift bursts have been suggested to belong to the "short"-hard category even though their durations are longer than 5 seconds. They are GRB 050911 with $T_{90} \sim 16 \mathrm{~s}$ (Page et al. 2006a; Berger et al. 2006a) and GRB 051227 with $T_{90} \sim 8 \mathrm{~s}$ (Barthelmy et al. 2006). These three GRBs are also listed in Table 1 as Type I GRBs (marked with '*' symbol), making the total number in the sample 15. In the rest of the paper, I will interchangeably use "Type I / Type II" and "short (hard) / long (soft)" in the text.

It is worth commenting that afterglow modeling also lends indirect support to the merger scenario of Type I GRBs. The immediate environment of Type I GRBs should be tenuous with low ISM density (Panaitescu et al. 2001; Perna \& Belczynski 2002; Fan et al. 2005a). As a result, afterglow observations (especially multi-wavelength) may potentially lead to constraints on the density and thereby shed light onto the nature of the bursts. Afterglow modeling has been indeed carried out for several short GRBs, and the results are generally consistent with a low ambient medium density (e.g. Fan et al. 2005a; Panaitescu 2006a; Roming et al. 2006b; Burrows et al. 2006). Some short GRBs appear "naked" (i.e. no external shock afterglow component) or completely with no afterglow detection. They are also consistent being born in a low-density medium. On the other hand, abnormal afterglow behaviors have been observed. For example, GRB 060313 exhibited complex structure with different decay indices and flaring (Roming et 

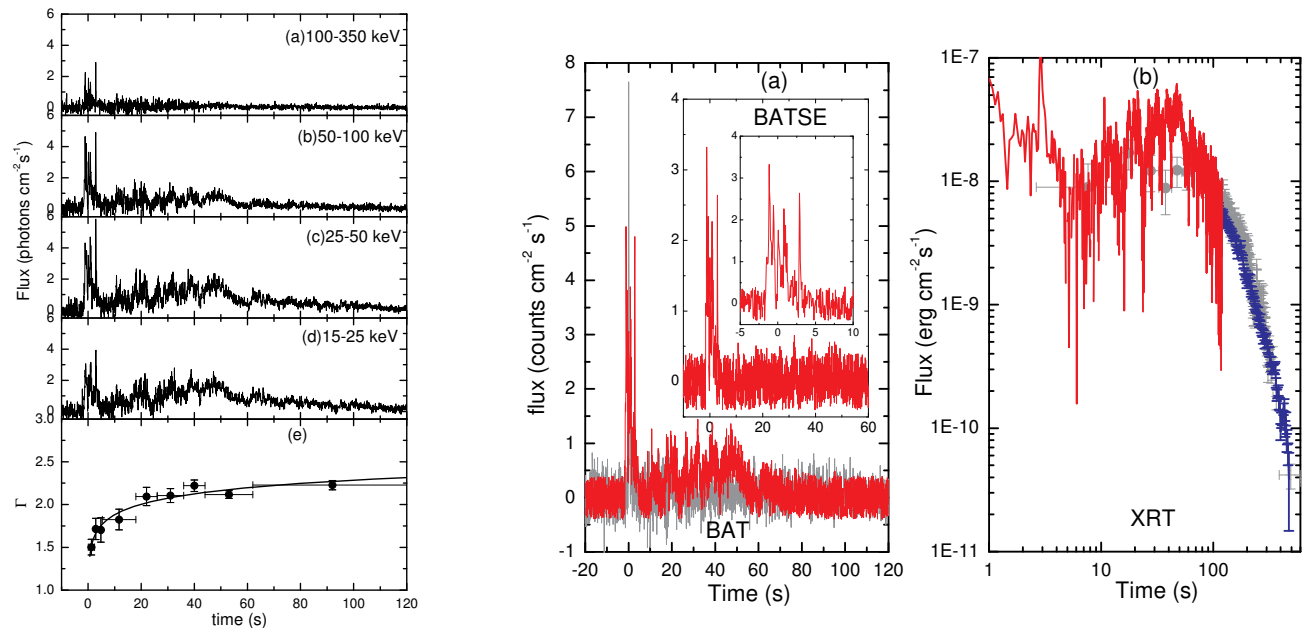

Fig. 2 The peculiar burst GRB 060614 (Gehrels et al. 2006; Zhang et al. (2007a). left: Multi-wavelength lightcurves; Right: The gamma-ray and X-ray properties of the "pseudo" burst appears similar to GRB 050724 (Zhang et al. 2007a).

al. 2006b). The optical flux fluctuation may be related to density fluctuation of the ambient medium, or to weak central engine activities. Another caveat is that a low density medium may not be solely associated with Type I GRBs. When analyzing GRB radiative efficiency of a sample of Swift bursts (most are Type II bursts), Zhang et al. (2007b) found that in about $1 / 3$ of the bursts the X-ray band is below the cooling frequency for a very long period of time. This suggests a very small $\epsilon_{B}$ or a very low ambient density for long GRBs as well.

As for cosmological setting, luminosity and redshift distributions of known short-hard bursts have been used to constrain the progenitor lifetime of compact star binaries. With a small sample of short GRBs with known redshift, Nakar et al. (2006a) and Guetta \& Piran (2006) found that in order to reproduce the observed redshift distribution, the typical progenitor lifetime is typically longer than previously believed, and the local burst rate is also higher than previous believed. On the other hand, Belczynski et al. (2006) argue a bimodal distribution of the merger times corresponding to two distinct evolutionary tracks of compact star binaries. They argue that there exist a population of mergers whose merger time scale is short, so that they could be found in star-forming galaxies. Zheng \& Ramirez-Ruiz (2006) study the merger rate in both early-type and late-type galaxies and argue a large merger time for at least half of short GRBs. The existence of some possible high- $z$ short GRBs (e.g. Levan et al. 2006a; Berger et al. 2006b) may suggest that there exist some fast evolutionary channels such as those proposed by Belczynski et al. (2002, 2006). Nakar et al. (2006a) have tested several possible function forms for the lifetime distribution. The high- $z$ short GRBs suggest that the narrow lognormal distribution tested by Nakar et al. is inconsistent with the data, while the wide lognormal or a single power law function forms may be still consistent with the data.

Another interesting question regarding short-hard GRBs is what fraction is produced by SGR giant flares in nearby galaxies. The Dec.27, 2004 giant flare event from SGR 1800-20 has a luminosity $(3.7 \pm 0.9) \times 10^{46} d_{15}^{2} \mathrm{erg} \mathrm{s}^{-1}$ (where $d_{15}$ is the distance of the source in unit of $15 \mathrm{kpc}$ ), suggesting a high detectability of similar events up to $30 d_{15} \mathrm{Mpc}$, which would contribute to a significant fraction of BATSE short-hard bursts (Hurley et al. 2005). A search for associations 
of well-localized short GRBs with nearby galaxies (Nakar et al. 2006b), however, sets an upper limit of this fraction of $\sim 15 \%$. Schaefer (2006) found that most BATSE short hard GRBs were not associated with moderately bright nearby galaxies. Tanvir et al. (2005) on the other hand, report a correlation between the locations of previously observed short bursts and the positions of galaxies in the local universe, indicating that about $10 \%-25 \%$ short GRBs originate at $z<0.025$.

\subsection{GRBs vs. XRFs}

X-ray flashes (XRFs) are the extension of typical long GRBs to the softer, and fainter regime. They were first identified with the Beppo-SAX satellite (Heise et al. 2001; Kippen et al. 2002), and studied more extensively with HETE-2 in the pre-Swift era (e.g. Sakamoto et al. 2005; Lamb et al. 2005a; D'Alessio et al. 2006). The lightcurves of XRFs are similar to those of long GRBs, with rapid temporal variability in many cases (Heise et al. 2001; D'Alessio et al. 2006). The spectral properties of XRFs are similar to those of GRBs, except that the values of the peak energy $E_{p}$ of the burst $\nu F_{\nu}$ spectrum are much smaller (Cui et al. 2005). The peak flux and the total energy fluence of XRFs are also correspondingly smaller (Sakamoto et al. 2005; D'Alessio et al. 2006). There is no clear separation between GRBs and XRFs. Bursts in the grey zone are sometimes called "X-ray rich GRBs" (XRRs). Besides the traditional $E_{p}$-distribution peak around $200 \mathrm{keV}$ (Preece et al. 2000), there is tentative evidence of a second distribution peak of $E_{p}$ around $15 \mathrm{keV}$ (Liang \& Dai 2004). The poor statistics however does not allow a robust claim of two distinct components in $E_{p}$-distribution. It is possible that the GRB-XRF $E_{p}$ distribution forms a broad peak around $100 \mathrm{keV}$.

Since the identification of XRFs, many suggestions have been proposed to interpret XRFs and their relation with GRBs. In general these models fall into two broad categories: i.e. XRFs differ from GRBs extrinsically (different distances or different viewing angles) or intrinsically (different physical parameters, different radiation mechanisms, or even different progenitors and central engines). The following is a list of models of XRFs proposed previously. The first four are "extrinsic" models, while the latter five are "intrinsic" ones. Swift has detected and extensively monitored a handful of XRFs. These observations significantly constrained the possible models of XRFs.

- High redshift GRBs. One early speculation (Heise et al. 2001) is that XRFs are distant GRBs so that the redshift effect makes them softer and fainter. Redshift measurements of several GRBs (e.g. $z=0.251$ for XRF 020903, Soderberg et al. 2004; $z=0.21$ for XRF 040701, Soderberg et al. 2005) suggest that at least some of them are nearby events. Analyses of the $E_{p}$ predictions in various GRB prompt emission models suggest that $E_{p}$ are usually functions of many parameters (including $z$ ), and that the dependences on some other parameters (e.g. the bulk Lorentz factor $\Gamma$ ) are more sensitive than the dependence on $z$ (e.g. Table 1 of Zhang \& Mészáros 2002c). This suggests that redshift should not be the sole factor to define an XRF. A systematic study of the redshift distribution of XRFs rules out the suggestion that XRFs are high- $z$ GRBs (Gendre et al. 2006a).

- Off-beam viewing geometry for a uniform GRB jets. The energy budget requirement and the temporal breaks in some afterglows have led to the suggestion that GRB ejecta are beamed (Rhoads 1997, 1999; Sari et al. 1999; Frail et al. 2001) ${ }^{2}$. The simplest model (not necessarily the most realistic model) suggests that the jet forms a uniform conical structure

\footnotetext{
${ }^{2}$ It is worth emphasizing that the salient-feature of the jet model, i.e. an achromatic temporal break in multi-wavelength afterglows, has not been generally confirmed after two years of Swift operation (see e.g. Willingale et al. 2006; Burrows 2006; Zhang 2007; Covino et al. 2006, and §3.3, §5 for more discussion).
} 
with sharp edge in energy. Within this scenario, it has been suggested that GRBs correspond to on-beam geometry while XRFs correspond to off-beam geometry (e.g. Yamazaki et al. 2002, 2004a). A direct prediction of such a scenario is that the early lightcurve should rise initially due to the gradual entrance of the main ultra-relativistic cone into the observer's field of view (Granot et al. 2002). Recent Swift observations suggest that the afterglows are decaying from the very early epoch of the observation (e.g. Schady et al. 2006b; Mangano et al. 2007a). This essentially rules out a sharp-edge off-beam geometry of XRFs. In some XRFs, the early decay slope is shallow. However, an early shallow decay is a common feature of most Swift GRB X-ray afterglows (see $\S 3.1$ for more discussion). This model may be amended by introducing a smoothed edge, which is effectively a structured jet as discussed below.

- Off-axis viewing geometry for a (one-component) structured jet. GRB jets may have significant structure, with an angle-dependent energy per solid angle and possibly Lorentz factor as well (Mészáros et al. 1998). An on-axis geometry of a structured jet would modify the afterglow temporal decay rate (Mészáros et al. 1998; Dai \& Gou 2001; Panaitescu 2005a), while an off-axis geometry would mimic a jet-break-like lightcurve as the Lorentz factor along the line-of-sight is reduced to be comparable to the viewing angle from the jet axis (Rossi et al. 2002; Zhang \& Mészáros 2002b; Wei \& Jin 2003; Kumar \& Granot 2003; Granot \& Kumar 2003; Panaitescu \& Kumar 2003; Salmonson 2003; Rossi et al. 2004). Within such a picture, energy per solid angle decreases with viewing angle with respect to the jet axis. Depending on the unknown jet structure, at certain viewing angles, an otherwise detected GRB (if viewed near the jet axis) would be observed as an XRF. The jet angular structure is unknown, and in reality it may not follow any simple analytical function. For the purpose of modeling, usually power law jets $\left(\epsilon(\theta) \propto \theta^{-k}\right.$, and in particular $k \sim 2$, Rossi et al. 2002; Zhang \& Mészáros 2002b) and Gaussian jets $\left(\epsilon(\theta) \propto \epsilon_{0} \exp \left(-\theta^{2} / 2 \theta_{0}^{2}\right)\right.$, Zhang \& Mészáros 2002b) have been widely discussed. Both models have been suggested to interpreted XRFs (e.g. Zhang et al. [2004a] for Gaussian jets and Jin \& Wei [2004] and D'Alesio et al. [2006] for power law jets). Lamb et al. (2005a) pointed out that an $\epsilon(\theta) \propto \theta^{-2}$ structured jet tends to over predict the number of XRFs, which is inconsistent with the rough 1:1:1 number ratio for GRBs, XRRs and XRFs. The Gaussian jets can easily pass this and several other observational constraints (Zhang et al. 2004a, X. Dai \& Zhang 2005). Since there are relativistically moving materials (though with a smaller energy) along the line of sight, the lightcurve in this model decays from the very beginning (Kumar \& Granot 2003; Salmonson 2003), not inconsistent with the observational constraint from X-ray lightcurves (Mangano et al. 2007a). Yamazaki et al. (2004b) introduced "patches" or "mini-jets" in a Gaussian-like structured jets to present a unified model of long, short GRBs as well as XRRs and XRFs. As discussed in $§ 2.1$, it is now clear that short GRBs form a distinct new population from long GRBs, XRRs and XRFs, which cannot be unified within this model.

- Two-component jets. Another widely discussed model is the two-component jet model, a special type of structured jets. Because this model is motivated physically by progenitor models, it receives broad attention. In the collapsar model of Type II GRBs, it is natural to expect a hot cocoon surrounding the central relativistic jet that penetrates from the star (Woosley et al. 1999; Zhang et al. 2003b, 2004b; Mészáros \& Rees 2001; Ramirez-Ruiz et al. 2002a; Mizuta et al. 2006; Morsony et al. 2006). The cocoon would form a distinct second less energetic jet component. Even in the naked GRB models, a neutron-rich MHD outflow would be naturally separated into a narrow, high- $\Gamma$ proton jet and a wide, low- $\Gamma$ neutron jet (Vlahakis et al. 2003; Peng et al. 2005). In both types of model, if the line of sight sweeps the less-energetic wide beam, one may observe an XRF. Phenomenologically, the two-component jet model has been introduced in several other contexts (Lipunov et al. 
2001; Berger et al. 2003a). In particular, within the XRF context, Huang et al. (2004) have interpreted the rising bump of the optical lightcurve of XRF 030723 within the framework of the two-component jet model. An alternative interpretation of the bump is the supernova component (Tominaga et al. 2004). The tentative bi-modal distribution of $E_{p}$ of GRBs and XRFs was also suggested as a support to the two-component jet model (Liang \& Dai 2004). Swift has followed some XRFs extensively to very late epochs. The lightcurve of XRF 050416A (Mangano et al. 2007a) keeps decaying with a constant slope to very late times. This greatly constrains the two-component jet model for XRFs: the narrow bright jet component must not be prominent enough to leave a rebrightening signature in the lightcurve. This suggests that at least two components are not required to interpret XRFs. Similar conclusions are drawn from the observations of other XRFs (e.g. Levan et al. 2006b).

- Intrinsically faint, less collimated jets. Lamb et al. (2005a) suggested a toy model invoking varying opening angle of jets. While GRBs are bright, narrow jets, XRFs are much fainter, wider jets. Starting with the assumption of constant energy reservoir of all events (as derived by Frail et al. 2001; Bloom et al. 2003 - but not confirmed by more systematic observations by Swift, e.g. Willingale et al. 2006; Burrows 2006; Zhang 2007), they drew the conclusion that GRBs have a typical opening angle of about 1 degree, while XRFs are essentially isotropic events. These narrow opening angles for GRBs are inconsistent with the typical angle derived from the afterglow jet break data (typically 5 degrees, see Zhang et al. 2004a for more discussion). On the other hand, if the standard energy reservoir assumption is dropped (as is suggested by the recent Swift data), this very narrow jet inference is no longer valid. The constant-slope power law decay lightcurve of XRF 050416A (Mangano et al. 2007a) is consistent with a wide-beam jet model. It is however worth commenting that the constant-slope power law decay of X-ray lightcurves seem to be a common feature of some other normal GRBs as well (e.g. 100 day lightcurve of GRB 060729, Grupe et al. $2006 \mathrm{~b}$ ), so that the jet opening angle may not be the crucial criterion to define whether a burst is a GRB or an XRF.

- Dirty fireballs. A naive expectation is that bursts with lower Lorentz factors would receive smaller Doppler boost and therefore give softer, fainter emission. These dirty fireballs (e.g. Dermer et al. 1999; Huang et al. 2002) have been suggested as the origin of XRFs. A detailed study of $E_{p}$ models (Zhang \& Mészáros 2002c) suggest that depending on prompt emission models, $E_{p}$ depends on $\Gamma$ in a non-trivial way. In particular, the popular internal shock model predicts high $E_{p}$ 's for dirty fireballs. The dirty fireball suggestion is in any case relevant for the external shock model and models invoking internal magnetic field dissipation or photosphere dissipation (Table 1 of Zhang \& Mészáros 2002c). Current Swift XRT observations strongly suggest an internal origin of GRB prompt emission (Zhang et al. 2006, see $\S 3.1$ for more discussion). So the dirty fireball suggestion for XRFs, if proven true, may suggest internal dissipation models other than the conventional internal shock models. It is interesting to note that the latest extreme XRF 060218 discovered by Swift (Campana et al. 2006a) shows an extremely long and smooth lightcurve with very low $E_{p} \sim 5 \mathrm{keV}$. Along with other intermediate XRFs (e.g. Lamb et al. 2005a; Sakamoto et al. 2005, 2006a), XRF 060218 also satisfies the $E_{p} \propto E_{\text {iso }}^{1 / 2}$ Amati-relation (Amati et al. 2002; Amati et al. 2006). Furthermore, a study of the multi-band temporal profiles and spectral lags of XRF 060218 suggests that its spectral lags are extremely long (Liang et al. 2006b), roughly consistent with the luminosity - lag relation discovered in BATSE long GRBs (Norris et al. 2000) and confirmed by Swift Type II GRBs (Gehrels et al. 2006). All these seem to be consistent with the straightforward intuition that XRF 060218 is a less-Lorentz-boosted burst comparing with canonical GRBs, suggesting a dirty fireball. The low Lorentz factor for XRF 060218 is also inferred from radio observations (Soderberg et 
al. 2006a) and is required in theoretical models to interpret this peculiar event (Dai et al. 2006b; Wang et al. 2006a; Toma et al. 2007). Another comment is that the dirty fireball suggestion does not exclude the structured jet and wide opening angle jet model discussed above, since in those models the Lorentz factors along the line of sight could be also low. On the other hand, there is no evidence for off-axis emission for XRF 060218. Another low-luminosity nearby GRB 980425 has been extensively monitored in radio at late times, which significantly constrained the off-axis model for the burst (Waxman 2004a).

- Intrinsically inefficient GRBs from clean fireballs. This is a specific XRF model within the framework of the internal shock model (Barraud et al. 2005), since in this model $E_{p} \propto \theta_{p}^{4} L^{1 / 2} r^{-1}$ (e.g. Zhang \& Mészáros 2002c), where $\theta_{p}$ is the internal energy of the protons in the internal shocks, $L$ is the wind luminosity, and $r \sim \Gamma^{2} c \delta t$ is the internal shock radius, and $\delta t$ is the variability time scale. A clean fireball (large $\Gamma$ ) tends to give a larger internal shock radius, at which magnetic field strength is smaller so that the typical synchrotron frequency $\left(E_{p}\right)$ is lower. An inefficient internal shock reduces $\theta_{p}$, and also helps to lower $E_{p}$. Indeed in the pre-Swift era it has been found that XRFs have lower radiative efficiency than GRBs by comparing their prompt emission energy with the late time kinetic energy inferred from the X-ray afterglow data (e.g. Soderberg et al. 2004; Lloyd-Ronning \& Zhang 2004). However, using the earliest Swift data, it is found that XRFs are generally as efficient as GRBs (Schady et al. 2006a; Zhang et al. 2007b), suggesting that XRFs are not intrinsically inefficient GRBs. The apparent low efficiency derived from the late time X-ray data may be caused by a prolonged energy injection epoch in the early phase. This seems to be consistent with the expectation of the off-axis structured (e.g. Gaussian-like) jet model of XRFs (Zhang et al. 2004a).

- Photosphere-dominated emission models. In GRB fireballs, there are in principle three emission regions that could potentially contribute to the observed prompt gammaray emission. Besides the traditional external shock (Rees \& Mészáros 1992; Mészáros \& Rees 1993) and internal shocks (Rees \& Mészáros 1994), baryonic and pair photospheres (Thompson 1994; Mészáros \& Rees 2000; Kobayashi et al. 2002; Mészáros et al. 2002; Rees \& Mészŕos 2005; Ryde 2005; Ramirez-Ruiz 2005; Ryde et al. 2006; Thompson et al. 2006) are another important emission site. The domination of photosphere emission, under certain conditions, could give rise to soft emission that characterizes XRFs. The data generally suggest that the $E_{p}$ distribution of GRBs and XRFs forms a broad distribution peak. As a result, to accept the photosphere interpretation of XRFs (Mészáros et al. 2002), one should expect that the prompt emission of GRBs is also from the photosphere. Such a model is being advocated recently (Rees \& Mészáros 2005; Ryde et al. 2006; Thompson et al. 2006).

- Completely different origin with respect to GRBs. The last possibility is that XRFs are different from GRBs. They may originate from different progenitors, may have different central engines, and different radiation mechanisms as well. As discussed above, XRFs seem to be a natural extension of GRBs to softer and fainter regime. If the difference is not due to the viewing angle effect, the variation of progenitor and central engine properties must be gradual and smooth, and probably without abrupt transition. The recent observations of XRF 060218, however, raise new discussion on the topic. The radio afterglow observation of XRF 060218 (Soderberg et al. 2006a) suggests that the central engine may be a neutron star rather than a black hole. A similar conclusion was reached independently by modeling of the supernova associated with the XRF (Mazzali et al. 2006). A population study suggests that the low-luminosity GRBs such as XRF 060218 may require a distinct new component in the GRB luminosity function (Liang et al. 2006c). The existence of the thermal X-ray component in the prompt emission spectrum (Campana et al. 2006a) may require a novel radiation mechanism different from that for canonical GRBs (e.g. Wang et al. 2006). The 
facts that XRF 060218 satisfy the Amati-relation (Amati et al. 2006) and the lag-luminosity relation (Liang et al. 2006b), on the other hand, suggest that the radiation mechanism for XRFs should not be much different from that for GRBs (For more discussion on the two correlations, see §3.4). It is worth emphasizing that bursts with different progenitor systems and central engines could well share the same radiation mechanism, since the fireball properties are generic and independent of the unknown central engine.

In summary, Swift observations have significantly narrowed down the possible models of $\mathrm{XRFs}$. The high-redshift scenario is essentially ruled out. The sharp edge off-beam jet model is disfavored by the early afterglow data of XRFs. Observations of XRF 060218 (the softest XRF) suggest that its radiation mechanism should not be very different from that of GRBs. The longterm constant-slope decay of XRF 050416A (Mangano et al. 2007a) disfavors jet models other than the wide-beam uniform jet model and a large viewing angle Gaussian-like structured jet model. Regarding the Lorentz factor, in view of the properties of XRF 060218 (long pulse, long lag, soft spectrum), the dirty (rather than clean) fireball scenario is favored. Combining with the fact that GRB prompt emission is likely of internal origin (Zhang et al. 2006), mechanisms other than internal shocks are preferred. Photosphere or magnetic dissipation mechanisms may be good candidates.

An interesting question is how XRFs are related to the X-ray flares observed following some GRBs and XRFs (Burrows et al. 2005b). Could it be possible that XRFs are simply Xray flares without prompt emission detection? As discussed later (§3.1.2), X-ray flares generally require reactivation of the central engine, and therefore would have the same energy dissipation mechanism as the prompt emission. On the other hand, observationally the lightcurves of XRFs are more variable than X-ray flares, which are similar to those of prompt GRBs (Heise et al. 2001; D'Alessio et al. 2006). This may suggest that prompt XRF emission is related to the prompt accretion phase at the central engine, while X-ray flares are related to accretion at late epochs, which generally predicts a smoother lightcurve consistent with the viscous disk evolution at large radii (e.g. Perna et al. 2006; Proga \& Zhang 2006).

\subsection{HL-GRBs vs. LL-GRBs}

The detection of XRF 060218 at $z=0.0331$ (Mirabal et al. 2006) within 1.5 years of operation of Swift (Campana et al. 2006a), together with the previous detection of GRB 980425 at $z=0.0085$ by BeppoSAX (Galama et al. 1998), suggest that the local event rate of low-luminosity (LL) GRBs is very high. The volume enclosed by $z<0.033$ is very small, $V_{z<0.033} \sim 0.01 \mathrm{Gpc}^{3}$. One can naively estimate the local event rate of these low-luminosity (LL) GRBs $\left(\rho_{0}^{\mathrm{LL}}\right)$ by $\rho_{0}^{\mathrm{LL}} V_{z<0.033}\left(T^{\text {Bepp }} \Omega^{\text {Bepp }} / 4 \pi+T^{\text {Swift }} \Omega^{\text {Swift }} / 4 \pi\right) \sim 2$, where $T^{\text {Bepp }} \sim 6 \mathrm{yr}$ and $T^{\text {Swift }} \sim 1.5 \mathrm{yr}$ are the operation times for the BeppoSAX and Swift missions, respectively, and $\Omega^{B e p p} \sim 0.123$ and $\Omega^{\text {Swift }} \sim 1.33$ are the solid angles covered by the two missions, respectively. This rough estimate gives $\rho_{0}^{\mathrm{LL}} \sim 800 \mathrm{Gpc}^{-3} \mathrm{yr}^{-1}$, which is much greater than the local event rate of the conventional high-luminosity (HL) GRBs of $1 \mathrm{Gpc}^{-3} \mathrm{yr}^{-1}$ (e.g. Schmidt 2001) and its simple extrapolation to low luminosities, i.e. $\leq 10 \mathrm{Gpc}^{-3} \mathrm{yr}^{-1}$ (Guetta et al. 2004). Such a high event rate for LL-GRBs has been independently derived by several groups (e.g. Cobb et al. 2006a; Pian et al. 2006; Soderberg et al. 2006a; Liang et al. 2006c). By investigating the 1-D and 2-D distributions of luminosity and redshift for a sample of GRBs with known redshifts, Liang et al. (2006c) found that the current sample is not compatible with a single luminosity function component. Rather, data require a distinct LL-GRB component other than the HL-component (Fig.3). The former component has a much higher event rate than the latter. In view that GRB 060218's central engine may be a neutron star rather than a black hole (Mazzali et al. 2006; Soderberg et al. 2006a), one would speculate that the apparent bimodal distribution in the 

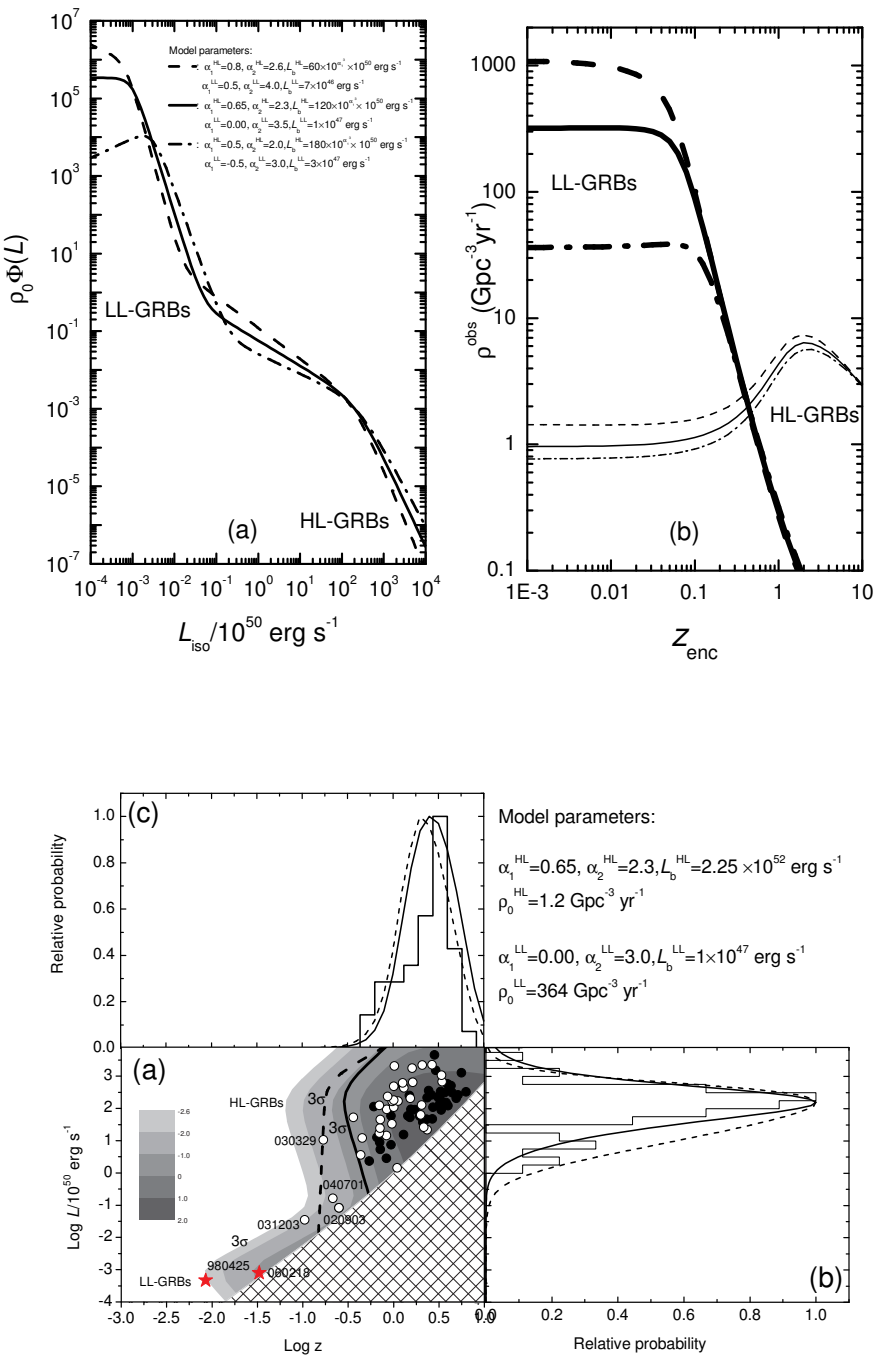

Fig. 3 The HL and LL populations of GRBs. Upper: The suggested two-component luminosity function and predicted observed local event rate as a function of redshift; Lower: The model can interpret the observed 2-D luminosity and redshift distributions of HL and LL GRBs (from Liang et al. 2006c).

luminosity function may be related to the two distinct types of central engines involved, e.g. HL-GRBs involve black holes, while LL-GRBs involve neutron stars.

Although these individual LL-GRBs are less energetic and under-luminous, due to their much higher event rate, they could give an interesting contribution to various diffuse emission backgrounds. For example, assuming LL-GRBs produce gamma-rays in internal shocks similar to HL-GRBs, the protons in LL-GRBs would produce high energy neutrinos through photo- 
meson interaction at the $\Delta$-resonance, which have the dominant contribution at energies above $10^{16} \mathrm{eV}$ (Gupta \& Zhang 2007a; Murase et al. 2006).

\subsection{Optically bright vs. dark, optically luminous vs. dim}

In the previous optical follow up observations, GRBs are generally divided into two categories, optically bright and optically dark ones (e.g. Jakobsson et al. 2004; Rol et al. 2005). The latter typically account for $\sim 50 \%$ of the total population ${ }^{3}$. The discovery of the early optical flash of GRB 021211 (Fox et al. 2003b; Li et al. 2003a) in the HETE-2 era had led to the speculation that as long as observations are performed early enough, most dark bursts are not dark. However, Swift UVOT did not detect a large number of bursts even at very early epochs (Roming et al. 2006a). It is not straightforward to define an optically "dark" burst. Jakobsson et al. (2004) and Rol et al. (2005) use the criterion that a burst is "darker" than it is expected to be (using spectral extrapolation from the X-ray band for example) to define a dark burst. This might be the most quantitative method to define late darkness. At early times, it is somewhat "expected" theoretically to observe optical emission originated from the reverse shock (which is not a strong contributer to the X-ray band). In such a case, the X-ray band and the optical band are not from a same emission component, rendering the quantitative definition inconclusive. The existence of X-ray flares (and possible other internal-related emission in the X-ray band) makes the case even more complicated (see e.g. the completely different early X-ray/optical lightcurves in GRB 060418 and GRB 060607A, Molinari et al. 2006). The non-detection of a large fraction of Swift bursts by UVOT (Roming et al. 2006a) at least suggest that the reverse shock component is insignificant. Among the other possible reasons of optical darkness, foreground extinction, circumburst absorption, and high redshift are the best candidates.

Among the optically bright GRBs, it is intriguing to discover that there are two sub-classes, namely optically luminous and optically dim categories (Liang \& Zhang 2006a; Nardini et al. 2006; Kann et al. 2006). The rest-frame lightcurves of GRBs with known redshifts are found to follow two "universal" tracks. The rest-frame 10-hour luminosities of the bursts with known redshifts show a clear bimodal distribution. The optically dim bursts all appear to be located at redshifts lower than $\sim 1$ and their lightcurve tends to be smooth and single-pulsed, while the optically luminous bursts have a wider redshift distribution and the lightcurves are more complex (Liang \& Zhang 2006a). A related dichotomy in the prompt emission properties (lags and internal luminosity functions) was identified by Hakkila \& Giblin (2006, see also Norris 2002). The origin of the dichotomy is unknown. The two universal tracks of afterglow lightcurves may be related to the different total explosion energies involved in the two groups of bursts. It is worth commenting that Gendre \& Boer (2005) have reported two groups of X-ray afterglow lightcurves. However, this is not confirmed by the Swift data (O'Brien et al. 2006; Willingale et al. 2006). The lack of evidence in X-rays to support the bimodal optical luminosity distribution is puzzling. On the other hand, there is growing evidence that some early X-ray afterglow emission may be more related to the GRB central engine, and hence, is a different component from the optical one (which is likely from the external shock). This might be the reason of the discrepancy.

\section{PHYSICS OF GRBS AND AFTERGLOWS}

The standard GRB fireball model has been extensively reviewed (Piran 1999; Mészáros 2002; Zhang \& Mészáros 2004; Lu et al. 2004; Piran 2005; Mészáros 2006). Regardless of the nature

\footnotetext{
${ }^{3}$ Swift UVOT does not detect optical afterglows for $\sim 67 \%$ of the Swift bursts. Combining with ground-based follow ups, the non-detection rate is $\sim 45 \%$ (P. Roming, 2006, private communication).
} 
of the explosion, the generic fireball shock model invokes a relativistically expanding ejecta. According to this model, the ejecta is intrinsically intermittent and unsteady, and is composed of many mini-shells with a wide range of bulk Lorentz factors. Internal shocks (Rees \& Mészáros 1994) are likely developed before the global fireball is decelerated by the ambient medium, which are generally believed to be the emission sites of the observed prompt GRB emission. Alternatively, magnetic dissipation may be responsible for the prompt gamma-ray emission even without internal shocks. The fireball is decelerated at a larger distance after sweeping enough interstellar medium whose inertia becomes noticeable, and the blastwave enters a self-similar deceleration regime at later times (Blandford \& McKee 1976). Upon deceleration, a pair of shocks forms. A long-lived forward shock propagating into the ambient medium gives rise to the long-term broad band afterglow (Mészáros \& Rees 1997a; Sari et al. 1998); and a short-lived reverse shock propagating into the ejecta itself gives rise to a possible optical/IR flash and a radio flare (Mészáros \& Rees 1997a, 1999; Sari \& Piran 1999a,b). The relativistic ejecta are likely collimated (Rhoads 1999; Sari et al. 1999), and the jets may have substantial angular structures (Zhang \& Mészáros 2002b; Rossi et al. 2002). This general theoretical framework has been successful to interpret most of the observational data in the pre-Swift era. With the successful launch and operation of Swift, we now have unprecedented information about GRB afterglows, which sheds light on many outstanding problems in the pre-Swift era (Zhang \& Mészáros 2004 for a summary): e.g. central engine, composition and geometric configuration of the GRB fireball, and its interaction with the ambient medium.

It is informative to clarify the definitions of "prompt emission" and "afterglow" at this point. Traditionally, "prompt emission" refers to the emission component detected by the gammaray detector (sometimes also optical emission simultaneously detected during the gamma-ray emission phase); while all the emissions detected by other instruments at later times are termed as "afterglow". On the other hand, Swift observations strongly suggest that such a definition scheme is not physical. X-ray flares, if strong and hard enough, would be included as part of prompt emission (e.g. by comparing GRB 050724 and GRB 060614, Zhang et al. 2007a). Physically, it is more meaningful to define emission components as of "internal" (central engine) or "external" (medium) origins. In such a scheme, the central engine related emission likely extends to much later epochs and can no longer be defined as "prompt". In the following discussion, I will still stick to the conventional terminology, but will discuss the distinct physical meanings of various emission components.

\subsection{A canonical X-ray afterglow lightcurve}

One of the major discoveries of Swift is the identification of a canonical X-ray afterglow behavior (Nousek et al. 2006; Zhang et al. 2006; O'Brien et al. 2006b; Chincarini et al. 2005, see Fig.4). Besides the prompt emission phase (denoted as ' 0 '), there are a total of five components in the $\mathrm{X}$-ray lightcurves. Not every burst has all five components, so that their lightcurves may vary from one another. In any case, their afterglow lightcurve components could be generally fit into this generic picture. The five components are:

- Steep decay phase (I): Typically smoothly connected to the prompt emission (Tagliaferri et al. 2005; Barthelmy et al. 2005c), with a temporal decay slope $\sim-3$ or steeper (sometimes up to -10, e.g. Vaughan et al. 2006; Cusumano et al. 2006b; O'Brien et al. 2006b) extending to $\sim\left(10^{2}-10^{3}\right)$ s. Usually with a different spectral slope from the later afterglow phases ${ }^{4}$.

4 The steep decay component has been observed in two BeppoSAX bursts: GRB 990510, Pian et al. 2001 and GRB 010222, in't Zand et al. 2001. It was Swift that reveals that this is a common feature among bursts. 

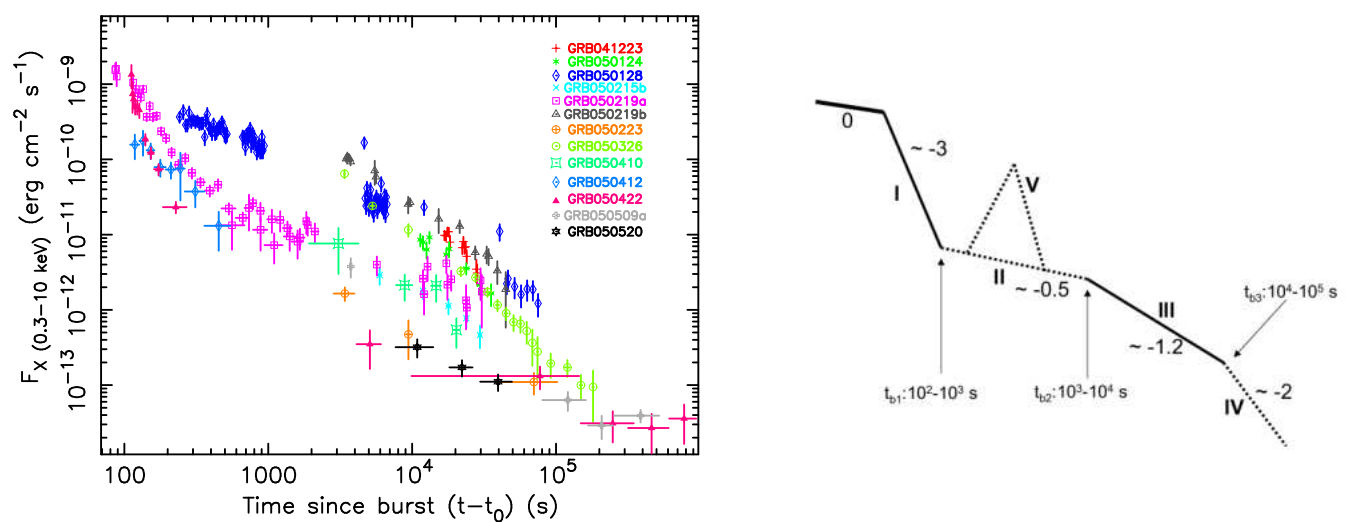

Fig. 4 A canonical X-ray afterglow lightcurve revealed by Swift XRT observations. Left: Data (from Nousek et al. 2006); Right: A cartoon picture (from Zhang et al. 2006).

- Shallow decay phase (II): Typically with a temporal decay slope $\sim-0.5$ or flatter extending to $\sim\left(10^{3}-10^{4}\right) \mathrm{s}$, at which a temporal break is observed before the normal decay phase (e.g. Campana et al. 2005; De Pasquale et al. 2006a). There is no spectral evolution across the break.

- Normal decay phase (III): Usually with a decay slope $\sim-1.2$, and usually follows the predictions of the standard afterglow model (Mészáros \& Rees 1997a; Sari et al. 1998; Chevalier \& Li 2000). A systematic test of the afterglow closure-relations (e.g. Table 1 of Zhang \& Mészáros 2004) suggests, however, that a fraction of bursts do not satisfy any afterglow model (Willingale et al. 2006).

- Post Jet break phase (IV): Occasionally observed following the normal decay phase, typically with a decay slope $\sim-2$, satisfying the predictions of the jet model.

- X-ray flares (V): Appear in nearly half of GRB afterglows. Sometimes multiple flares appear in one GRB. Typically have very steep rising and decaying slopes (Burrows et al. 2005b; Falcone et al. 2006; Romano et al. 2006a) with $\delta t / t \ll 1$. Appear in both longduration (Falcone et al. 2006) and short-duration GRBs (Barthelmy et al. 2005b; Campana et al. 2006b), and both GRBs and XRFs (Romano et al. 2006a).

Except for the normal decay and the jet-break phases, all the other three components were not straightforwardly expected in the pre-Swift era ${ }^{5}$. As of the time of writing, the steep decay phase and X-ray flares are better understood, while the shallow decay phase is still a mystery.

\subsubsection{Steep decay phase: tail of the prompt emission}

The generally accepted interpretation of the steep decay phase is the tail emission due to the so-called "curvature effect" (Fenimore et al. 1996; Kumar \& Panaitescu 2000; Dermer 2004; Zhang et al. 2006; Panaitescu et al. 2006a; Dyks et al. 2005, for discussion of the curvature effect in the prompt gamma-ray phase, see e.g. Kocevski et al. 2003; Shen et al. 2005; Qin \& Lu 2005; Qin et al. 2006a). The basic assumption of this interpretation is that the GRB emission

5 The flare-like signature was seen by Beppo-SAX, but it was interpreted as the onset of the afterglow (Piro et al. 2005). More detailed theoretical calculations (Lazzati \& Begelman 2006; Kobayashi \& Zhang 2007) suggest that the onset of afterglow cannot produce a sharp lightcurve feature to interpret X-ray flares. 
region is disconnected from the afterglow region (the external shock), and that the emission from the GRB emission region ceases abruptly. This is consistent with the conjecture of internal shocks or other internal dissipation mechanisms (e.g. photosphere dissipation, magnetic field reconnection, etc). Since it is generally assumed that the ejecta has a conical geometry, the curvature of the radiation front causes a propagation delay for high-latitude emission from the line of sight. Combining with the variation of the Doppler factor at different latitudes, one gets a simple prediction $\alpha=2+\beta$ for the emission outside the $\Gamma^{-1}$ emission cone, where the convention $F_{\nu} \propto t^{-\alpha} \nu^{-\beta}$ is adopted. The salient feature of this interpretation is that it could be directly tested since both $\alpha$ and $\beta$ could be measured directly from the observational data, given that two complications are treated properly (Zhang et al. 2006): First, for internal emissions, every time when the central engine restarts, the clock should be re-set to zero ${ }^{6}$. In a $\log -\log$ lightcurve, this usually introduces an "artificial" very steep decay if the GRB trigger time (which is usually taken as $t=0$ ) significantly leads the time zero point $\left(t_{0}\right)$ of the corresponding emission episode. Second, the observed decay is the superposition of the curvature effect decay and the underlying afterglow decay from the external shock. One needs to subtract the underlying afterglow contribution before performing the test. The credibility of the curvature effect interpretation is that by properly taking into account the two effects mentioned above, the steep decay is consistent with $\alpha=2+\beta$ with $t_{0}$ shifted to the beginning of the last pulse of prompt emission (Liang et al. 2006a) at least in some cases.

Besides the standard curvature effect model, other interpretations for the steep decay phase have been discussed in the literature.

- In some cases, the steep-decay slope may be shallower than the expectation of the curvature effect ${ }^{7}$. This would suggest that the emission in the shock region may not cease abruptly, but rather decay (cool) with time gradually, leading to a decaying internal shock afterglow (Fan \& Wei 2005; Zhang et al. 2006).

- Yamazaki et al. (2006) study the curvature effect of an inhomogeneous fireball (mini-jets). They found that the decay tail is generally smooth, but sometimes could have structures, which may interpret the small-scale structure in some of the decay tails.

- Pe'er et al. (2006b) suggest that the emission from the relativistically expanding hot plasma "cocoon" associated with the GRB jet could also give rise to the steep decay phase observed by Swift.

Motivated by the discovery of the spectrally evolving tails in GRB 050724 (Campana et al. 2006a) and GRB 060614 (Gehrels et al. 2006; Zhang et al. 2007a; Mangano et al. 2007b), recently Zhang et al. (2007c) performed a systematic time-dependent spectral analysis of 17 bright steep decay tails. They found that while 7 tails show no apparent spectral evolution, the other 10 do. A simple curvature effect model invoking an angle-dependent spectral index cannot interpret the data. This suggests that the curvature effect is not the sole factor to control the steep decay tail phase at least in some bursts. Zhang et al. (2007c) show that some of the spectrally evolving tails might be interpreted as superposition of the curvature effect tail and an underlying "central engine afterglow", which is soft but decays "normally". Such a component has been seen in GRB 060218 (Campana et al. 2006a), which cannot be interpreted by the standard external shock afterglow model and may be from a decaying central engine (Fan et al. 2006). The strong spectral evolutions in GRB 050724, GRB 060218, and GRB 060614,

${ }^{6}$ We notice that for external shock related emissions, taking the GRB trigger time as the time zero point is generally required (Lazzati \& Begelman 2006; Kobayashi \& Zhang 2007).

7 It is worth noticing that generally a decay slope steeper than the curvature effect prediction is not allowed, unless the jet is very narrow. Usually, even if the intrinsic temporal decay slope is steeper than $2+\beta$, the curvature effect nonetheless takes over to define the decay slope. 
however, cannot be interpreted with such a model. They may be interpreted as cooling of the internal-shocked region (Zhang et al. (2007c).

It is interesting to notice that in some bursts (e.g. GRB 050421, Godet et al. 2006; GRB 050911, Page et al. 2006a) the X-ray afterglow is dominated by the steep decay component (with overlapping X-ray flares). Such naked GRBs may be surrounded by a very tenuous medium so that the external shock component is very faint.

\subsubsection{X-ray flares: restarting the central engine}

The X-ray flares have the following observational properties (Burrows et al. 2005b; Falcone et al. 2006; Romano et al. 2006a; Chincarini et al. 2007, Burrows et al. 2007; see Fig.5): Rapid rise and fall times with $\delta t / t_{\text {peak }} \ll 1$; many light curves have evidence for the same decaying afterglow component before and after the flare; multiple flares are observed in some bursts with similar properties; large flux increases at the flares; typically degrading fluence of flares with time, but in rare cases (e.g. GRB 050502B) the flare fluence could be comparable with that of the prompt emission; flares soften as they progress; and later flares are less energetic and more broadened than early flares. These properties generally favor the interpretation that most of them are not associated with external-shock related events. Rather they are the manifestations of internal dissipations at later times, which requires restarting the GRB central engine (Burrows et al. 2005b; Zhang et al. 2006; Fan \& Wei 2005; Ioka et al. 2005; Wu et al. 2005a; Falcone et al. 2006; Romano et al. 2006a; Lazzati \& Perna 2007) ${ }^{8}$. Compared with the external shock related models, the late internal dissipation models have the following two major advantages (Zhang et al. 2006): First, since the clock needs to be re-set each time when the central engine restarts, it is very natural to explain the very sharp rising and falling lightcurves of the flares. Second, energetically the late internal dissipation model is very economical. While in the refreshed external shock models a large energy budget is needed (the injection energy has to be at least comparable to that already in the blastwave in order to have any significant injection signature, Zhang \& Mészáros 2002a), the internal model only demands a small fraction of the prompt emission energy to account for the distinct flares.

The leading candidate of the late internal dissipation model is the late internal shock model. In such a model, the collisions could be between the fast shells injected later and the slow shells injected earlier during the prompt phase (e.g. Zou et al. 2006; Staff et al. 2006) or between two shells injected at later times (see Wu et al. 2005a for a categorization of different types of collisions). One concern is whether later collisions between two slow shells injected during the prompt phase could give rise to the observed X-ray flares. This is generally not possible. In order to produce late internal shocks, the two slow shells must both have a low enough Lorentz factor so that at the time of collision they do not collide with the decelerating blastwave. Also in order not to collide with each other earlier, their relative Lorentz factor $\Delta \Gamma$ must be very small. When they collide, the internal energy is usually too small to give rise to significant emission. Should such a collision occur, most likely it has no interesting observational effect (Lazzati \& Perna 2007; Zhang 2007). Generally, in the internal shock model the observed time sequence reflects the time sequence in the central engine (Kobayashi et al. 1997). As a result, the observed X-ray flares $\left(10^{2}-10^{5}\right)$ s after the prompt emission must imply that the central engine restarts during this time span, say, typically thousands of seconds but could be as late as days after the prompt emission is over.

\footnotetext{
8 It had been questioned whether well-separated gamma-ray pulses are due to restarting of the central engine or inhomogeneity within the central engine outflow in the pre-Swift era (e.g. Ramirez-Ruiz et al. 2001), but the case was inconclusive.
} 

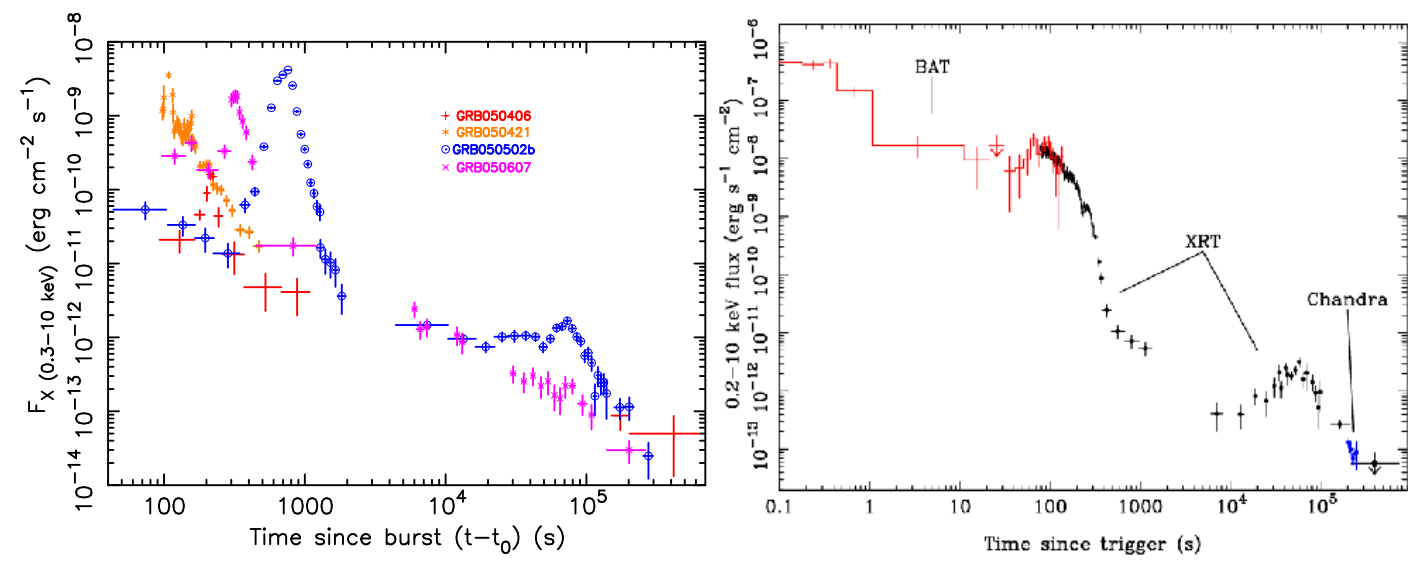

Fig. 5 Lightcurves show erratic X-ray flares. Left: Several long GRBs including the giant flare of 050502B (from Nousek et al. 2006); Right: Flares following the short GRB 050724 (from Barthelmy et al. 2005b).

The late internal dissipation model of X-ray flares is also tested by Liang et al. (2006a). The same logic of testing the steep decay component is used. The starting assumption is that the decay of X-ray flares are controlled by the curvature effect after the abrupt cessation of the internal dissipation, so that $\alpha=2+\beta$ is assumed to be valid. After subtracting the underlying forward shock afterglow contribution, Liang et al. (2006a) searched for the valid zero time points $\left(t_{0}\right)$ for each flare to allow the decay slope to satisfy the requirement of the curvature effect model. If the hypothesis that flares are of internal origin is correct, $t_{0}$ should be generally before the rising segment of each flare. The testing results are impressive: Most of the flares indeed have their $t_{0}$ at the beginning of the flares. This suggests that the internal dissipation model is robust for most of the flares. It is worth emphasizing that even the late slow bump at around 1 day following the short GRB 050724 (Barthelmy et al. 2005b; Campana et al. 2006b) satisfies the curvature effect model, suggesting that the central engine is still active even at 1 day after the trigger. This is also consistent with the late Chandra observation of this burst (Grupe et al. 2006a) that indicates that the afterglow resumes to the pre-flare decay slope after the flare.

Having identified the correct model for the flare phenomenology, one is asked about a fundamental question: how to restart the central engine. No central engine models in the preSwift era have specifically predicted extended activities far after the prompt emission phase. Prompted by the X-ray flare observations, the following suggestions have been made recently, and none is proven by robust numerical simulations yet at the moment.

- Fragmentation or gravitational instabilities in the massive star envelopes. King et al. (2005) argued that the collapse of a rapidly rotating stellar core leads to fragmentation. The delayed accretion of some fragmented debris after the major accretion event leads to X-ray flares following collapsar-related GRBs.

- Fragmentation or gravitational instabilities in the accretion disk. Observations of the short GRB 050724 (Barthelmy et al. 2005b; Campana et al. 2006b; Grupe et al. 2006a) reveal that it is also followed by several X-ray flares starting from 10 s of seconds all the way to $\sim 10^{5} \mathrm{~s}$. The properties of these X-ray flares are similar to those in long GRBs. The requirement that both long and short GRBs should produce X-ray flares with similar properties prompted Perna et al. (2006) to suggest that fragmentation in the accretion disk, 
the common ingredient in both long and short GRB models, may be the agent for episodic accretion that powers the flares.

- Magnetic barrier around the accretor. Based on MHD numerical simulations in other contexts (e.g. Proga \& Begelman 2003) and theoretical arguments, Proga \& Zhang (2006) argued that a magnetic barrier near the black hole may act as an effective modulator of the accretion flow. The accretion flow can be intermittent in nature due to the role of magnetic fields. This model does not require the flow being chopped (e.g. due to fragmentation or gravitational instabilities) at larger radii before accretion, although in reality both processes may occur altogether. The magnetic barrier model is in accordance with the magnetic origin of X-ray flares based on the energetics argument (Fan et al. 2005d).

- NS-BH merger. Flares in GRB 050724 (Barthelmy et al. 2005b) pose a great challenge to the previous compact star merger models. Numerical simulation of NS-NS mergers typically gives a short central engine time scale $(0.01-0.1) \mathrm{s}$, if the final product is a $\mathrm{BH}$-torus system (Aloy et al. 2005). In order to account for the late time flares in 050724, Barthelmy et al. (2005b) suggest a possible NS-BH merger progenitor system. Numerical simulations of BH-NS merger systems have been performed. Although X-ray flares at 100s of seconds or later still challenge the model, extended accretion over several seconds could be produced (Faber et al. 2006; Shibata \& Uryu 2006; cf. Rosswog 2005). Lately Rosswog (2006) shows that if materials are launched into eccentric orbits during a compact binary coalescence, the fallback of these materials would last long enough to power X-ray flares hours after the coalescence.

- NS-NS merger with a postmerger millisecond pulsar. Dai et al. (2006a) argued for a possible solution of the extended X-ray flares following NS-NS merger GRBs. Numerical simulations have shown that the product of a NS-NS merger may not be a BH (Shibata et al. 2005), if the NS equation-of-state is stiff. Instead, the final product may be a differentiallyrotating massive neutron star. If the initial magnetic fields of the NS is not strong, the $\alpha-\Omega$ dynamo action would induce magnetic explosions that give rise to late internal shocks to produce X-ray flares thousands seconds after the trigger (Dai et al. 2006a). Earlier discussion on $\alpha-\Omega$ dynamo within the GRB context can be found in (Thompson \& Duncan 1993; Kluzniak \& Ruderman 1998; Ruderman et al. 2000; Rosswog et al. 2003). Price \& Rosswog (2006) suggest transient superstrong magnetic fields during mergers through numerical simulations.

- Multi-stage central engine. Gao \& Fan (2006) and Staff et al. (2006) proposed multistage central engine models to interpret X-ray flares.

- White dwarf - neutron star mergers. A related model is the WD-NS merger scenario revoked by King et al. (2007) as an effort to interpret "long" GRB 060614 without supernova associations. In view of the close analogy between GRB 060614 and GRB 050724 (Zhang et al. 2007a), this model can be relevant for X-ray flares following short GRBs.

Some other flare models have been discussed in the literature. The models that can only interpret one flare (e.g. the synchrotron self-inverse Compton in reverse shock, Kobayashi et al. 2007, and the companion model, MacFadyen et al. 2005) are found unattractive in view that multiple flares within a same burst seem to be common, and that the properties of the single flares are essentially the same as those of multiple flares. The suggestion that flares result from collisions of density clumps by the external shock (Dermer 2006) is so far not supported by numerical calculations. At least for clump angular sizes larger than $1 / \Gamma$, numerical calculations show very smooth features incompatible with the X-ray flare data (e.g. Zhang et al. 2006; Huang et al. 2006, 2007; Nakar \& Granot 2006). For smaller clumps (Dermer 2006), fast variability is possible, but current calculations fail to produce giant flares such as that in GRB 050502B (Falcone et al. 2006). Giannios (2006) interprets multiple X-ray flares as delayed magnetic dissipation in a 
decelerating Poynting-flux dominated jet without introducing reviving the central engine. It is however not clear how to interpret the clear $t_{0}$-resetting of flares as discovered by Liang et al. (2006a).

\subsubsection{Shallow decay phase: still a mystery}

The shallow decay phase could follow the steep decay phase or immediately follow the prompt emission (O'Brien et al. 2006b; Willingale et al. 2006). In most cases, a same shallow decay phase is detected in the optical band as well (e.g. Mason et al. 2006). This component is very likely related to the external shock. However, the very origin of this shallow decay phase is more difficult to identify, since there exist several different possibilities that are not easy to differentiate among each other from the X-ray observations. Generally the spectral index does not change across the temporal break from the shallow decay phase to the normal decay phase (but some slight change occurs in some bursts, see Willingale et al. 2006). This essentially rules out the models that invoke crossing of a spectral break across the band. The nature of the break should be then either hydrodynamical or geometrical.

The following models have been discussed in the literature.

- Energy injection invoking a long-term central engine. The most straightforward interpretation of the "shallower-than-normal" phase is that the total energy in the external shock continuously increases with time. This requires substantial energy injection into the fireball during the phase (Zhang et al. 2006; Nousek et al. 2006; Panaitescu et al. 2006a). There are two possible energy injection schemes (Zhang et al. 2006; Nousek et al. 2006). The first one is to simply invoke a long-lasting central engine, with a smoothly varying luminosity, e.g. $L \propto t^{-q}$ (e.g. Zhang \& Mészáros 2001a). In order to give interesting injection signature $q<1$ is required; otherwise the increase of the total energy in the blastwave is negligible. Such a possibility is valid for the central engines invoking a spinning-down pulsar (Dai \& Lu 1998a,b; Zhang \& Mészáros 2001a) or a long-lasting BH-torus system (MacFadyen et al. 2001). One possible signature of this scenario that differentiates it from the varying- $\Gamma$ model discussed below is a strong relativistic reverse shock, if at the shock interacting region the $\sigma$-parameter (the ratio between the Poynting flux and the kinetic flux) is degraded to below unity (Dai 2004; Yu \& Dai 2006). Alternatively, if $\sigma$ is still high at the shock region, the reverse shock may be initially weak, but would still become relativistic if the engine lasts long enough (i.e. this is effectively a rather thick shell, Zhang \& Kobayashi 2005). The observational data suggest a range of $q$ values with a typical value $q \sim 0.5$. This is different from the requirement of the analytical pulsar model $(q=0)$. However, numerical calculations suggest that a pulsar model can fit some of the XRT lightcurves (Fan \& Xu 2006; De Pasquale et al. 2006b; Yu \& Dai 2006).

- Energy injection from ejecta with a wide $\Gamma$-distribution. This model invokes a distribution of Lorentz factor of the ejecta with the low- $\Gamma$ ejecta lagging behind the high- $\Gamma$ ones, which pile up onto the blastwave when the high- $\Gamma$ part is decelerated (Rees \& Mészáros 1998). In order to produce a smooth power law decay, the $\Gamma$-distribution needs to be close to a power law with $M(>\Gamma) \propto \Gamma^{-s}$. A significant energy injection requires $s>1$. The temporal break around $\left(10^{3}-10^{4}\right) \mathrm{s}$ suggests a cutoff of Lorentz factor around several tens, below which $s$ becomes shallower than unity (Zhang et al. 2006). Granot \& Kumar (2006) have used this property to constrain the ejecta Lorentz factor distribution of GRBs within the framework of this model. The reverse shock of this scenario is typically non-relativistic (Sari \& Mészáros 2000), since the relative Lorentz factor between the injection shell and the blastwave is always low when the former piles up onto the latter. 
- Delayed energy transfer to the forward shock. Analytically, the onset of afterglow is estimated to be around $t_{d e c}=\max \left(t_{\gamma}, T\right)$, where $t_{\gamma} \sim 5 \mathrm{~s}\left(E_{\mathrm{K}, 52} / n\right)^{1 / 3}\left(\Gamma_{0} / 300\right)^{-8 / 3}(1+z)$ is the time scale at which the fireball collects $\Gamma^{-1}$ of the rest mass of the initial fireball from the ISM, and $T$ is the duration of the explosion. The so-called "thin" and "thick" shell cases correspond to $t_{\gamma}>T$ and $t_{\gamma}<T$, respectively (Sari \& Piran 1995; Kobayashi et al. 1999). Numerical calculations suggest that the time scale before entering the BlandfordMcKee self-similar deceleration phase is long, of order several $10^{3} \mathrm{~s}$ (Kobayashi \& Zhang 2007). This suggests that it takes time for the kinetic energy of the fireball to be transferred to the medium. In a high- $\sigma$ fireball, there is no energy transfer during the propagation of a reverse shock (Zhang \& Kobayashi 2005). Although energy transfer could happen after the reverse shock disappears, this potentially further delays the energy transfer process Detailed numerical simulations are needed to verify this. The shallow decay phase may simply reflect the slow energy transfer process from the ejecta to the ambient medium. This model (e.g. Kobayashi \& Zhang 2007) predicts a significant curvature of the lightcurves. This is consistent with some of the lightcurves that show an early "dip" before the shallow decay phase. For those cases with a straight shallow decay lightcurve, one needs to invoke superposition of the rising lightcurve and the steep decay tail to mimic the observations.

- Off-beam jet model. Geometrically one can invoke an off-beam jet configuration to account for the shallow decay. Eichler \& Granot (2006) show that if the line of sight is slightly outside the edge of the jet that generates prominent afterglow emission, a shallow decay phase can be mimicked by the combination of the steep decay GRB tail. However, the expectation of this model is the correlations among the slow decay slope, hump luminosity and its epoch, which are not confirmed observationally (Panaitescu 2006b). Toma et al. (2006) discussed a similar model within the framework of the patchy jet models.

- Two-component jet model. A geometric model invoking two jet components to produce two-component afterglows could also fit the shallow-decay data, since additional free parameters are invoked (Granot et al. 2006; Jin et al. 2006; Panaitescu 2006b).

- Precursor model. Ioka et al. (2006) suggest that if there is a weak precursor leading the main burst, a shallow decay phase can be produced as the main fireball sweeps the remnants of the precursor.

- Varying microphysics parameter model. One could also invoke evolution of the microphysics shock parameters to reproduce the shallow decay phase (Ioka et al. 2006; Fan \& Piran 2006a; Granot et al. 2006; Panaitescu et al. 2006b).

- Dust scattering model. Shao \& Dai (2006) suggest that small angle scattering of X-rays by dust could also give rise to a shallow decay phase under certain conditions.

- Cannonball model. Dado et al. (2006) explains the canonical X-ray afterglow lightcurve within the framework of the cannonball model, which invokes a series of different radiation mechanisms to explain different segments of the lightcurves.

- Central engine afterglow. Finally, it remains possible that the shallow decay phase is not related to the external shock, but is due to a long-lived central engine activity. In such a case, the X-ray emission and the optical emission may be two distinct components.

Can different possibilities be differentiated by more abundant data? It seems to be a challenging task. I am inclined to the first three interpretations on the above list. For the two energy injection models, one expects different reverse shock signatures (i.e. relativistic reverse shock for the long-term central engine model and non-relativistic reverse shock for the varying- $\Gamma$ model). This would give different radio emission properties at early times. On the other hand, the uncertainty of the composition of the central engine outflow (e.g. the $\sigma$ parameter) would make the reverse shock signature of the former model more obscured. The delayed energy transfer model (the third one on the above list) is the simplest. If it is correct, the so-called shallow 
decay phase is nothing but a manifestation of the onset of afterglow (Kobayashi \& Zhang 2007). The peak time can be then used to estimate the bulk Lorentz factor of the fireball (which is $\sim 100$ for standard parameters). This might be the case for at least some of the bursts.

\subsection{Optical, IR \& radio afterglows}

In the pre-Swift era, the afterglow observations were mainly carried out in the optical and radio bands. The late time optical/radio observations have been focused on identifying temporal breaks in the lightcurves, which are generally interpreted as the "jet breaks" (see Frail et al. 2001; Bloom et al. 2003; Ghirlanda et al. 2004b; Dai et al. 2004; Friedman \& Bloom 2005; Liang \& Zhang 2005 for compilations of the jet break data in the pre-Swift era). Broad-band modeling was carried out for a handful of well observed bursts (Wijers \& Galama 1999; Panaitescu \& Kumar 2001, 2002; Yost et al. 2003), and the data are generally consistent with the standard external shock afterglow model. In some cases, very early optical flashes have been discovered (e.g. GRB 990123, Akerlof et al. 1999; GRB 021004, Fox et al. 2003a; GRB 021211, Fox et al. 2003b; Li et al. 2003a), which are generally interpreted as emission from the reverse shock (Sari \& Piran 1999a; Mészáros \& Rees 1999; Kobayashi \& Sari 2000; Kobayashi 2000; Wang et al. 2000; Fan et al. 2002; Kobayashi \& Zhang 2003a; Zhang et al. 2003; Wei 2003; Kumar \& Panaitescu 2003; Panaitescu \& Kumar 2004; Nakar \& Piran 2004). Early radio flares have been detected in a sample of GRBs (Frail et al. 2003), which are also attributed to the reverse shock emission (Sari \& Piran 1999a; Kobayashi \& Sari 2000; Soderberg \& Ramirez-Ruiz 2003). While optical robotic telescopes such as ROTSE indeed reported non-detections of optical early afterglows of some bursts, the general expectation for Swift before the launch has been that the UVOT would collect a good sample of early afterglow lightcurves to allow a detailed study of GRB reverse shocks.

In the Swift era, UVOT has been regularly collecting optical photons $\sim 100$ s after the burst triggers for most GRBs. Ground-based robotic telescopes (e.g. ROTSE-III, PAIRITEL, RAPTOR, P60, TAROT, Liverpool, Faulkes, KAIT, PROMPT, etc) have promptly observed most targets whenever possible. A good list of early optical detections have been made. However, the majority of bursts have very dim or undetectable optical afterglows (Roming et al. 2006a). This suggests that in most cases the reverse shock, if any, is not significant.

Figure 6(a) displays the theoretically predicted early optical afterglow lightcurves (Zhang et al. 2003) in the constant medium density (ISM) model $^{9}$. The thick solid line shows two peaks: the first peak followed by $\sim t^{-2}$ decay is the reverse shock emission peak time, which is typically at the shock crossing time $\left(t_{d e c}\right)$. The second peak followed by $\sim t^{-1}$ is the forward shock peak, which corresponds to the time when the typical synchrotron frequency $\nu_{m}$ crosses the optical band. Depending on parameters, the forward shock peak could be buried below the reverse shock component (the thin solid line). One therefore has two cases of optical flashes: rebrightening-type and flattening-type. A unified study of both reverse shock and forward shock emission suggests that the rebrightening lightcurves should be generally expected, if the shock microphysics parameters $\left(\epsilon_{e}, \epsilon_{B}, p\right.$, etc) are the same in both shocks. On the other hand, these microphysics parameters may not be the same in both shocks. In particular, if the central engine is strongly magnetized, as is expected in several progenitor models, the outflow likely carries a primordial magnetic field, which is likely amplified at the shocks. It is then possible to have $R_{B}=\left(\epsilon_{B, r} / \epsilon_{B, f}\right)^{1 / 2} \gg 1$ in some cases. This is actually the condition to realize the flattening-type lightcurves (Zhang et al. 2003). In order to interpret the bright optical flash and the subsequent flattening lightcurves in GRB 990123 and GRB 021211, one typically requires

\footnotetext{
9 For the model involving a stratified stellar wind medium, see Chevalier \& Li (2000); Wu et al. (2003); Kobayashi \& Zhang (2003b); Kobayashi et al. (2004); Zou et al. 2005.
} 

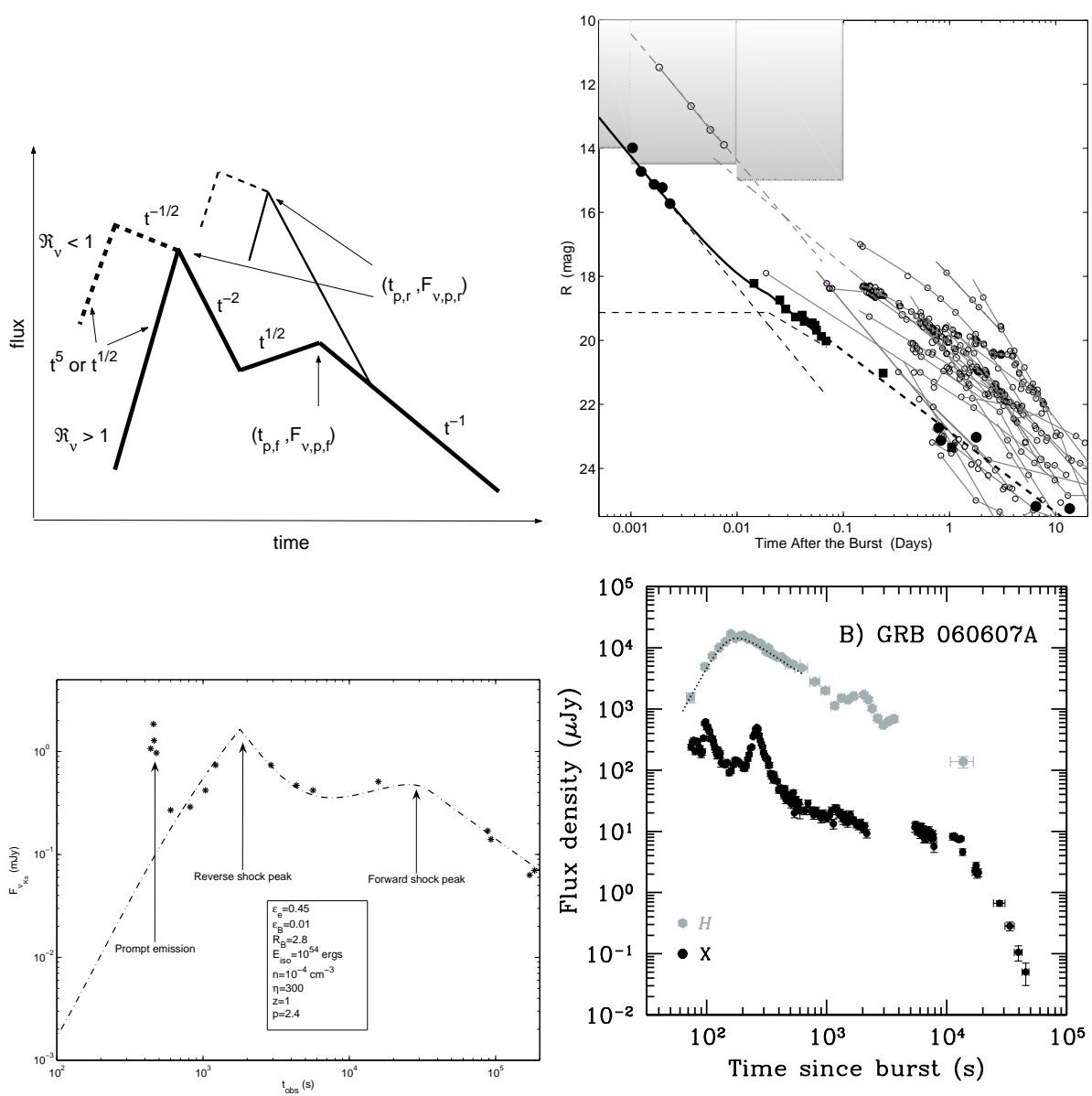

Fig. 6 Early optical afterglow lightcurves related to reverse and forward shock emission. (a) Top left: Theoretically expected early optical lightcurves, which show two types of behavior: flattening and rebrightening (from Zhang et al. 2003); (b) Top right: The flattening lightcurves detected from GRB 990123 and GRB 021211 (from Fox et al. 2003b); (c) Bottom left: The rebrightening lightcurve detected from GRB 041219A (data from Blake et al. 2005, model from Fan et al. 2005c); (d) Bottom right: The case that there is no evidence of reverse shock in GRB 060607A (from Molinari et al. (2006)).

$R_{B} \sim 10$ or more (Fan et al. 2002; Zhang et al. 2003; Kumar \& Panaitescu 2003; Panaitescu \& Kumar 2004). Besides GRB 990123, the flattening-type lightcurve was also detected for GRB 021211 (Fox et al. 2003b; Li et al. 2003, see Fig.6(b)).

The $\epsilon_{B}$ parameterization is based on a purely hydrodynamical treatment of shocks with magnetic fields put in by hand. Invoking a strong magnetic component in the reverse shock region raises the necessity to treat the dynamics more carefully with a dynamically important magnetic field. Zhang \& Kobayashi (2005) studied the reverse shock dynamics and emission for an outflow with an arbitrary $\sigma$ parameter. They found that the most favorable case for a bright optical flash (e.g. GRB 990123 and GRB 021211) is $\sigma \sim 1$, i.e. the outflow contains roughly equal amount of energy in magnetic fields and baryons. This is understandable: For a smaller $\sigma$, the magnetic field in the reverse shock region is smaller, and the synchrotron emission is 
weaker (see also Fan et al. 2004a). For a larger $\sigma$, the magnetic field is dynamically important, whose pressure dominates the outflow region. The shock becomes weak or does not exist at all when $\sigma$ is large enough.

The lack of bright optical flashes such as those observed in GRB 990123 and GRB 021211 is therefore not surprising. In order to have a bright flattening-type flash, one needs to by chance have an outflow with $\sigma \sim 1$, while both larger and smaller $\sigma$ 's would lead to not very significant optical flashes. Even without additional suppression effects, a non-relativistic shock with $\sigma=0$ would generally give a reverse shock peak flux below the forward shock peak level (Kobayashi 2000; Nakar \& Piran 2004; Zhang \& Kobayashi 2005). On the other extreme, a high- $\sigma$ flow would lead to very weak reverse shock emission or no reverse shock at all (Zhang \& Kobayashi 2005). Thus the tight early UVOT upper limits (Roming et al. 2006a) are not completely out of expectation. Additional mechanisms to suppress optical flashes have been discussed in the literature. Beloborodov (2005) argued that Compton cooling of electrons by the prompt $\mathrm{MeV}$ photons may be a way to suppress the optical flashes. Kobayashi et al. (2007) suggested that a dominant synchrotron-self-Compton process in the reverse shock region would suppress the synchrotron optical emission. Li et al. (2003b) and McMahon et al. (2006) suggested a pair-rich reverse shock with weak optical emission.

Despite of the general disappointments, several bright optical flashes have been detected in the Swift era, which could be generally interpreted within the reverse/forward shock model discussed above. The IR afterglow of GRB 041219A (Blake et al. 2005) is well modeled by a rebrightening lightcurve (Fan et al. 2005c) (see Fig.6(c)). Another flattening lightcurve was detected from GRB 060111B (Klotz et al. 2006). Marginal reverse shock signatures may be present in GRB 050525A (Blustin et al. 2006; Shao \& Dai 2005), GRB 050904 (Gendre et al. 2006b; Wei et al. 2006), GRB 060117 (Jelinek et al. 2006) and GRB 060108 (Oates et al. 2006). Data also suggest a second type of optical flashes, which tracks the gamma-ray lightcurves (for GRB 041219A, Vestrand et al. 2005). These optical flashes are likely related to internal shocks (Mészáros \& Rees 1999), probably neutron rich (Fan \& Wei 2004; Fan et al. 2005c, cf. Zheng et al. 2006). The time lags between the prompt gamma-ray and optical emission have been revealed in GRB 990123 and GRB 041219A (Tang \& Zhang 2006). In some cases (e.g. GRB 050820A, Vestrand et al. 2006), the contributions from both the tracking component and the external shock component are detected from the early optical lightcurve.

There are however cases that clearly show no reverse shock component at all in the early optical afterglows. GRB 061007 (Mundell et al. 2006; Schady et al. 2006a) is such a case. Reaching a peak magnitude $<11$ (similar to 9th magnitude of GRB 990123), both the X-ray and optical lightcurves show single power law decaying behavior from the very beginning $(\sim 80 \mathrm{~s}$ after the trigger). This suggests a strong external forward shock emission with enormous kinetic energy (Mundell et al. 2006) or a structured jet with very early jet break (Schady et al. 2006a). The reverse shock emission in this case is believed to peak at the radio band (Mundell et al. 2006). Molinari et al. (2006) recorded the densely-covered early optical afterglow lightcurves of GRB 060418 and GRB 060607A, which are both characterized by a round-shaped single bump that could be interpreted as the forward shock emission at the onset of afterglow. There is no evidence of a reverse shock component at all (Fig.6(d)). Among other possibilities, this is consistent with a high- $\sigma$ flow where the reverse shock is completely suppressed (Zhang \& Kobayashi 2005).

Wiggles and bumps have been observed in several pre-Swift GRB optical afterglows (e.g. GRB 021004, Holland et al. 2003; GRB 030329, Lipkin et al. 2004). Models to interpret these variabilities usually invoke external shock related processes, such as refreshed shocks, density fluctuation, inhomogeneous jets, or multiple component jets (Panaitescu et al. 1998; Zhang \& Mészáros 2002a; Lazzati et al. 2002; Heyl \& Perna 2003; Nakar et al. 2003; Berger et al. 2003a; 
Granot et al. 2003; Ioka et al. 2005). Early optical lightcurves may contain neutron decay signatures (Beloborodov 2003; Fan et al. 2005b). Kobayashi \& Zhang (2003a) interpreted the early fluctuations in GRB 021004 as a rebrightening lightcurve by combining both reverse and forward shock emission (see also Fan et al. 2005c for GRB 041219A). Ioka et al. (2005) pointed out that some optical fluctuations are difficult to interpret within any external shock related schemes, and they require reactivation of the central engine. That erratic X-ray flares generally require late central engine activities raises the question whether some optical flashes/flares are also due to the same origin (but softer and even less energetic, e.g. Zhang 2005). Recent optical afterglow observations reveal that "anomalous" optical afterglows seem to be the common feature (Stanek et al. 2007; Roming et al. 2006c). Although some of them could be accommodated within the external shock related models, some optical flares do show similar properties to X-ray flares (e.g. $\delta t / t<1$, Roming et al. 2006c), which demands late central engine activities. For example, the optical fluctuations detected in the short GRB 060313 optical afterglows (Roming et al. 2006b) may be better interpreted as due to late central engine activities than due to density fluctuations (e.g. Nakar \& Granot 2006). Efforts to model optical flares using the late internal shock model have been carried out recently (Wei et al. 2006; Wei 2007). The results suggest that for plausible parameters, even the traditional reverse shock optical flashes such as those in GRB 990123, GRB 041219A and GRB 060111B could be interpreted within the late internal shock model.

Due to a higher mean redshift of Swift bursts than that of pre-Swift bursts (Berger et al. 2005b; Jakobsson et al. 2006a), the efficiency to detect radio afterglows is lower in the Swift era. According to GCN Circular statistics (e.g. Greiner 2006), 17 radio afterglows were detected among about 200 GRBs detected by Swift in the first 2 years. Short-lived radio transients have been seen in some of these bursts (e.g. Soderberg et al. 2006c), some of which may be related to reverse shock emission (D. Frail, 2006, personal communication).

\subsection{Afterglow temporal breaks}

Temporal breaks (usually steepening breaks) have been commonly observed in broad-band afterglow lightcurves. The origin of these temporal breaks in multi-wavelength afterglows is still not well understood. Theoretically one expects the following four types of temporal breaks.

- Jet breaks. This is expected if the GRB outflow is collimated. The break occurs when the fireball is decelerated enough so that the relativistic beaming angle $1 / \Gamma(\Gamma$ is the bulk Lorentz factor of the fireball) becomes larger than the geometric collimation angle. At this time the observer starts to feel the energy deficit outside the jet cone. In the meantime, the jet starts to expand sideways due to a horizontally propagating sound wave. Both effects tend to steepen the lightcurve, and when combined together, result in a temporal break from a decay index $\sim-1$ to $\sim-2$ (Rhoads 1999; Sari, Piran \& Halpern 1999). For a structured jet, the argument still applies given that the jet opening angle is replaced by the observer's viewing angle (Zhang \& Mészáros 2002b; Rossi et al. 2002). Alternatively, cylindrical jets (Cheng et al. 2001) have been discussed in the literature. There are two distinct predictions for the jet model. (1) Because it is a pure hydrodynamical effect, the break must be achromatic, i.e. a temporal break should simultaneously occur in all wavelengths, including X-ray, IR/optical and radio. (2) The hydrodynamical effect should not affect the microscopic shock physics. The energy index of shock accelerated electrons should remain the same across the break, so should the photon index.

- Injection breaks. This is expected during the early phase of the afterglow when the total energy in the blastwave is still increasing with time. This could be due to either a long-lived GRB central engine (Dai \& Lu 1998a,b; Zhang \& Mészáros 2001) or a wide distribution 
of the ejecta Lorentz factor (Rees \& Mészáros 1998; Sari \& Mészáros 2000; Granot \& Kumar 2006). The break happens upon the sudden cessation of energy injection. Some "jet breaks" requiring $p<2$ may be also modeled by an injection break (Panaitescu 2005a). The temporal break separating the shallow decay component and the normal decay component in the canonical XRT early lightcurves is typically attributed to such an injection break (Zhang et al. 2006; Nousek et al. 2006; Panaitescu et al. 2006a). Since the process is again hydrodynamical, the break should be also achromatic. The spectral index across the break should remain the same, although a change of spectral index is not ruled out since the shock acceleration process may be altered when the injection ceases suddenly.

- Spectral breaks. This is the temporal break when a spectral break crosses the band. The typical spectral breaks include the characteristic synchrotron frequency $\nu_{m}$ and the cooling frequency $\nu_{c}$ (Sari et al. 1998). Alternatively, the accelerated electrons may have an intrinsic break in their energy spectrum (Li \& Chevalier 2001; Wei \& Lu 2002a). The crossing of the corresponding photon spectral break across the band would give rise to a distinct temporal break. Two predictions of this model are in distinct contrast to those of the previous two models. (1) The break must be chromatic, typically rolling from high energy bands (X-ray) to low energy bands (optical and radio) (but see otherwise, e.g. $\nu_{c}$ increases with time for the wind afterglow models, Dai \& Lu 1998c; Chevalier \& Li 1999; 2000); (2) the spectral indices before and after the break should be distinctly different (generally in a predictable way).

- Transrelativistic breaks. A steepening temporal break is expected at late times when an isotropic fireball turns from the highly relativistic phase to the non-relativistic phase (Wijers et al. 1997; Huang et al. 1998, 1999; Dai \& Lu 1999; Huang \& Cheng 2003). If the transition happens after the jet break, the transrelativistic break would be a flattening break (Livio \& Waxman 2000). Such a transition may have been observed in late radio afterglows (e.g. Frail et al. 2003), but there is no robust evidence that this break shows up in optical and X-ray lightcurves. The over 100-day follow-up observations of GRB 060729 (Grupe et al. 2006b) show a steady decay of the X-ray afterglow flux, suggesting that the transrelativistic phase happens at even later times at least for this burst. During the transition phase, the counter-jet beaming to the opposite direction may be detected through the observed excess radio emission (Li \& Song 2004).

Besides these breaks, more complicated lightcurves breaks may arise due to collisions between a late shell and the decelerating blast wave (Panaitescu et al. 1998; Zhang \& Mészáros 2002a; Granot et al. 2003), collisions between the blastwave and a density jump or a wind termination shock (e.g. Dai \& Lu 2002b; Dai \& Wu 2003; Ramirez-Ruiz et al. 2005; Pe'er \& Wijers 2006), etc.

The jet break interpretation has been generally accepted in the pre-Swift era ${ }^{10}$. This model could alleviate the energy budget problem encountered by some GRBs (e.g. GRB 990123, Kulkarni et al. 1999), and it is enhanced by the empirical relation that the geometrically corrected gamma-ray energy is quasi-standard (Frail et al. 2001; Bloom et al. 2003). By identifying the temporal break times as "jet break times" when $\Gamma^{-1}=\theta_{j}$ is satisfied (where $\Gamma$ is the bulk Lorentz factor and $\theta_{j}$ is the jet opening angle, Rhoads 1999; Sari et al. 1999, see Frail et al. 2001; Bloom et al. 2003; Ghirlanda et al. 2004b; Friedman \& Bloom 2005; Liang \& Zhang 2005 for compilations of the jet break data), one could infer the geometric configuration and the total energy budget of some bursts. In particular, several empirical relations related to afterglow temporal breaks have been discussed in the literature.

\footnotetext{
10 It has been doubted whether a jet model can indeed interpret the observed breaks, e.g. Wei \& Lu (2000b, 2002b).
} 
- Frail relation: Frail et al. (2001) and Bloom et al. (2003) found that the beaming-corrected gamma-ray energy is essentially constant, i.e. $E_{\gamma, i s o} \theta_{j}^{2}=E_{j} \sim$ const. Since the standard jet model predicts $t_{j} \propto E_{\gamma, i s o}^{1 / 3} \theta_{j}^{8 / 3}$ (Sari et al. 1999), this relation is generally consistent with $E_{\gamma, i s o} \propto t_{j}^{-1}$.

- Ghirlanda relation: Ghirlanda et al. (2004b) found that the beaming-corrected gammaray energy is not constant, but is related to the rest-frame spectral peak energy $\left(E_{p}\right)$ through $E_{p} \propto E_{\gamma, j}^{2 / 3}$. Again expressing $E_{\gamma, j}$ in terms of $E_{\gamma, i s o}$ and $t_{j}$, this relation is effectively $E_{p} \propto E_{\gamma, i s o}^{1 / 2} t_{j}^{1 / 2}$. Notice that the Ghirlanda relation and the Frail relation are incompatible with each other.

- Liang-Zhang relation: Liang \& Zhang (2005) took one step back. They discard the jet model, and only pursue an empirical relation among three observables, namely $E_{p}, E_{\gamma, \text { iso }}$ and the optical band break time $t_{b}$. The relation gives $E_{p} \propto E_{\gamma, i s o}^{0.52} t_{b}^{0.64}$. It is evident that if $t_{b}$ is interpreted as the jet break time, the Liang-Zhang relation is rather similar to the Ghirlanda relation. However, the former has the flexibility of invoking chromatic temporal breaks across different bands. So violating the Ghirlanda relation in other wavelengths (e.g. in the X-ray band, Sato et al. 2007) does not necessarily disfavor the Liang-Zhang relation.

- Willingale relation: Recently Willingale et al. (2006) performed a systematic study of the shallow-to-normal decay transition breaks in the early X-ray afterglows of a sample of Swift GRBs. By assuming they are jet breaks (the results actually suggest that they are not jet breaks), they found a new sequence of correlation which is parallel to the Ghirlanda relation. This is effectively a new series of $E_{p}-E_{\gamma \text {,iso }}-t_{b}$ relation as discussed by Liang-Zhang, but by replacing the optical breaks by X-ray breaks. The fact that the two correlations form a parallel sequence is intriguing.

The growing trend in the Swift era is that some breaks we see in the broad-band afterglows may not be jet breaks, and that the very origin of these breaks is still a mystery. This also raises the concern whether the pre-Swift "jet breaks" are indeed jet breaks. In fact, the "smokinggun" feature of the jet breaks, i.e. the achromatic behavior, was not robustly established in any of the pre-Swift bursts. The best case was GRB 990510 (Harrison et al. 1999), in which clear multi-color optical breaks were discovered, which are consistent with being achromatic. The radio data are also consistent with having a break around the same time. However, based on radio data alone, one cannot robustly fit a break time that is consistent with the optical break time (D. Frail, 2006, private communication). Most of other previous jet breaks were claimed using one-band data only, mostly in optical, and sometimes in X-ray or radio.

It has been highly expected that the multi-wavelength observatory Swift would clearly detect achromatic breaks in some GRBs to verify the long-invoked GRB jet scenario. The results are however discouraging. After detecting nearly 200 bursts, few "textbook" version jet breaks are detected. The lack of detections may be attributed partially to the intrinsic faintness of the Swift afterglows, and partially to the very low rate of late time optical follow-up observations. A higher average redshift also pushes required observations of already faint objects to even later observed times. Achromatic breaks were indeed observed in some bursts, but few satisfy the salient features expected in the jet model. For example, GRB 050801 (Rykoff et al. 2006) and GRB 060729 (Grupe et al. 2006b) have an early achromatic break covering both the X-ray and optical bands. However, the break is the transition from the shallow decay phase to the normal decay phase, which is likely an injection break rather than a jet break. GRB 050525A (Blustin et al. 2006) has an achromatic break in X-ray and optical bands, which might be interpreted as a jet break. However, the post-break temporal indices in both X-ray and optical bands are too shallow to comply with the $\propto t^{-p}$ prediction. An interesting case for a claimed achromatic jet break was GRB 060526 (X. Dai et al. 2007). However, the break indices before and after 
the break cannot be accommodated within the simple jet model (cf. Panaitescu 2006b). Maybe the best case is GRB 060614 (Mangano et al. 2007). An achromatic jet break around $100 \mathrm{ks}$ was seen by both the XRT in the X-ray band and by the VLT in the optical band. The post break temporal indices, although not identical, are similar to each other. Detailed modeling by Panaitescu (2006b) suggest that GRB 060124 may be also added to the jet break list.

In most other cases, data seem not to support the existence of jet breaks. The data also cast doubts on some of the previous identified jet breaks. These pieces of evidence are collected in the following.

- Optical follow up of GRB 060206 reveals a clear temporal break that would be regarded as a typical jet break should the X-ray have not been collected (Monfardini et al. 2006). However, X-ray data show a remarkable single power law decay without any evidence of a break at the optical break time (Burrows 2006). Notice that Stanek et al. (2007) reported a contaminating X-ray source, which may make the case less conclusive.

- Many other X-ray afterglows also show remarkable single power law decays extending to very late times (10 days or later, Burrows 2006). The lower limits of the beaming-corrected gamma-ray energy of many bursts already greatly exceed the standard energy reservoir value suggested by Frail et al. (2001) and Bloom et al. (2003) (Burrows 2006).

- Based on the Ghirlanda relation, Sato et al. (2007) have searched for expected jet breaks of three Swift bursts in the X-ray band with null results. The sample is expanded by Willingale et al. (2006) to seven. This suggests that Ghirlanda relation is not a common relation satisfied by most bursts. This fact however does not disfavor the Liang-Zhang relation, since an optical break may still exist at the expected time if the breaks are chromatic. Late time optical observations are needed to test whether the Liang-Zhang relation is generally valid/violated for most bursts.

- Covino et al. (2006) summarized the search of achromatic breaks of Swift afterglows using high-quality multi-wavelength data, and reported that no convincing case is identified.

It is worth mentioning that in several cases, the X-ray data are consistent with (not robustly suggest) having a jet break. These include GRBs 050315, 050814, 050820A, 051221A and 060428A (see Burrows 2006 for a review, also Panaitescu 2006b). In particular, late Chandra ToO observations of the short GRB 051221A reveal a possible jet break (Burrows et al. 2006). This, together with the achromatic jet break claimed for GRB 060614 (Mangano et al. 2007), suggest that at least some Type I GRBs are collimated.

The shallow-to-normal transition break in early X-ray afterglow lightcurves has been generally interpreted as injection breaks (see $\S 3.1 .3$ for more discussion). However, in some cases, clear chromatic features have been revealed (e.g. Fan \& Piran 2006a; Panaitescu et al. 2006b; Huang et al. 2007), which rejects the interpretation at least for those cases.

The data seem to suggest that there might exist other types of temporal breaks at least for some bursts that are not related to jet breaks and injection breaks. A very interesting feature of the afterglow breaks is that the X-ray breaks systematically lead the optical breaks, which in turn systematically lead the radio breaks. This fact, along with the chromatic breaks in both Xrays (e.g. Panaitescu et al. 2006b) and optical (e.g. Monfardini et al. 2006), drives Zhang (2007) to speculate an ad hoc scenario to interpret these temporal breaks as well as the Liang-Zhang and Willingale $\left(E_{\gamma, \text { iso }}-E_{p}-t_{b}\right)$ relations. In this scenario, the spectral break in the prompt gamma-ray emission $\left(E_{p}\right)$ and the chromatic temporal breaks in the afterglow lightcurves may be all related to the same electron energy distribution break that rolls down from high energy to low energy. Initially the break is in the gamma-ray band, which defines the $E_{p}$ in the prompt emission spectrum. Later this break moves to the X-ray band in $\sim\left(10^{3}-10^{4}\right) \mathrm{s}$, giving rise to the early injection-like breaks in some bursts. The break keeps rolling down to the optical band around a day, which can account for the pre-Swift optical breaks that were interpreted 
as jet breaks. Later it moves to the radio band in $\sim 10$ days. Such a scenario gives a natural link between $E_{p}$ and the optical and X-ray break times $t_{b}$ in the Liang-Zhang and Willingale relations, which is otherwise difficult to explain. (A similar scenario has been adopted by Wang et al. 2005 and Dai et al. 2005 to interpret the radio afterglow of the 2004, Dec.27 giant flare of SGR 1806-20.) The scenario has some difficulties (see Zhang 2007 for more discussion). The most severe one is that one expects changes of the spectral index across the breaks. In the X-ray band, this is not the case for most bursts, but there are still some cases that might satisfy the constraint (Willingale et al. 2006). No spectral change has been established in some optical breaks as well (e.g. Panaitescu 2005b). On the other hand, the scenario may be still valid for at least some bursts, and it is testable with broad-band densely-covered afterglow follow up observations. A hard test of this scenario is to find some bursts that have a break crossing through the X-ray, optical and radio bands in turn. Although no clear example is available in the Swift data sample, the previous GRB 030329 may satisfy the requirement of this model. It has been claimed that there are two "jet breaks" in this burst (Berger et al. 2003a): an early optical break and a later radio break. These two breaks were used to argue a two-component jet model for this burst. Within the scenario proposed here, the two breaks are simply the same break rolling over the optical and radio bands at different times. In view of the sequential relation between the Liang-Zhang and Willingale relations, a prediction of this scenario is that one would observe an "injection-like" break in the X-ray band first (say, thousands of seconds), and then detect a "jet-like" break in the optical band later (say, around a day), and a radio break at even later times (say, around 10 days). Whether or not such detections will be made would prove or falsify this ad hoc scenario (Zhang 2007). In such a scenario, the temporal breaks do not give us information about collimation and GRB energetics.

\subsection{Panchromatic observations \& prompt emission models}

The panchromatic, prompt observations of GRBs in the Swift era greatly advanced our understanding of GRB prompt emission.

Most of GRBs show an early steep decay tail (Tagliaferri et al. 2005; Goad et al. 2005; Barthelmy et al. 2005c). Interpreted as the curvature effect of high-latitude emission (see $\S 3.1 .1$ for discussion), this component suggests that prompt emission and afterglow are from distinct emission regions. This finally settles the internal vs. external shock debate of the prompt emission site (see Zhang et al. 2006 for more discussion). A small fraction of bursts do not show the steep decay phase (O'Brien et al. 2006b; Liang et al. 2006a; Willingale et al. 2006; Mundell et al. 2006; Schady et al. 2006a), so that the prompt emission and the early afterglow are smoothly connected together and the prompt emission might be of external origin as well. This might be due to an early deceleration of the fireball, likely due to a very large initial Lorentz factor and/or a dense medium.

Within the internal scenarios of the prompt emission, it is still unclear where the energy dissipation site (internal shocks, magnetic reconnection region, or baryonic and pair photosphere) is and what the radiation mechanism (synchrotron or jitter emission, inverse Compton or a combination of thermal and non-thermal emission components) would be. For discussion of internal prompt emission models, see e.g. Mészáros et al. (1994); Thompson (1994); Daigne \& Mochkovitch (1998); Pilla \& Loeb (1998); Medvedev \& Loeb (1999); Lloyd \& Petrosian (2000); Ghisellini et al. (2000); Panaitescu \& Mészáros (2000); Medvedev (2000); Mészáros \& Rees (2000); Spruit et al. (2001); Drenkhahn \& Spruit (2002); Mészáros et al. (2002); Zhang \& Mészáros (2002c); Dai \& Lu (2002a); Pe'er \& Waxman (2004a, 2005); Rees \& Mészáros (2005); Pe'er et al. (2005, 2006a); Ryde (2005); Ryde et al. (2006); Thompson et al. (2006), and Zhang \& Mészáros (2004) for a critical review. Due to the uncertainties inherited in the GRB jet 
composition and the degeneracy of models to interpret the limited prompt emission data, it has been a difficult task to identify the correct scenario for GRB prompt emission.

BAT is a narrow-band gamma-ray detector. For most of the Swift bursts, due to the narrow bandpass, it is difficult to precisely determine the prompt emission spectrum, especially the peak energy $E_{p}$. In most cases, the BAT spectrum can be only fitted by a simple power law (Zhang et al. 2007b). Nonetheless, by combining hardness ratio information (Cui et al. 2005), $E_{p}$ of the bursts could be estimated, which are generally consistent with those derived from joint BAT-Konus(WIND) fits (Zhang et al. 2007b). There is a rough correlation between the photon index $\Gamma$ and the derived $E_{p}$ (Zhang et al. 2007a,b; Sakamoto et al. 2006b), which can be used to roughly estimate $E_{p}$. For most bursts, Swift prompt emission observations do not provide more information than that gained in the BATSE era. Nonetheless, in rare cases Swift was triggered by a weak precursor (e.g. GRB 050117, Hill et al. 2006; GRB 060124, Romano et al. 2006b; and GRB 061121, Page et al. 2006b, see Burrows et al. 2007 for a review), so that all three instruments were targeted on the bursts during the prompt emission. This also happened for GRB 060218 whose prompt emission was long enough (Campana et al. 2006a). These panchromatic observations (Fig.7) unveil unprecedented spectral and temporal information of GRB prompt emission.

Statistically, the following empirical relations related to GRB prompt emission properties have been discovered in the pre-Swift era. Most of them are found still valid in the Swift era.

- Luminosity - spectral lag (Norris) relation: This relation suggests that more luminous bursts have shorter spectral lags $(\tau)$. For Type II (long-soft) GRBs, the relation reads $L_{\text {iso }} \propto \tau^{1.2}$ (Norris et al. 2000; Schaefer et al. 2001). This relation was confirmed by Swift bursts (Gehrels et al. 2006) including the peculiar long-soft GRB 060218 (Liang et al. 2006b). The interpretation of this relation is non-trivial from the first-principle prompt emission models. If one however assumes a standard radiation unit in the comoving frame, this relation may be simply related to a varying Doppler-boosting parameter among bursts (Salmonson 2000; Ioka \& Nakamura 2001; Norris 2002).

- Luminosity - variability (Fenimore-Reichart) relation: This relation suggests that more luminous bursts tend to have more variable lightcurves (Fenimore \& Ramirez-Ruiz 2000; Reichart et al. 2001). The scatter of this relation is large, and the index is subject to debate (e.g. Guidorzi et al. 2005; Reichart 2005; Guidorzi et al. 2006; Li \& Paczyński 2006). The definition of variability is also instrument-dependent. The origin of this relation may have something to do with the same kinetic effect to interpret the $L-\tau$ relation (Ioka \& Nakamura 2001) or the screening effect of the pair photosphere (Kobayashi et al. 2002; Mészáros et al. 2002).

- Amati and Yonetoku relations: Amati et al. (2002) discovered a simple relation $E_{p} \propto E_{\gamma, \text { iso }}^{1 / 2}$ with bursts with known redshifts (cf. Nakar \& Piran 2005; Band \& Preece 2005). Apparent outliers of the Amati relation include GRB 980425 and GRB 031203, but arguments (e.g. Ghisellini et al. 2006) suggest that they may not be intrinsic outliers should these events be detected by instruments like Swift (i.e. with a wider spectral coverage to detect prompt X-ray emission). A similar correlation has been noticed from the BATSE sample without redshift information (Lloyd et al. 2000). A related relation is $E_{p} \propto L_{p, i s o}^{1 / 2}$ (Yonetoku et al. 2004, see also Wei \& Gao 2003), where $L_{p, \text { iso }}$ is the isotropic peak luminosity. From the first-principle physical models, $E_{p}$ could be derived as functions of multiple unknown parameters, including the isotropic energy of the emitter and the unknown Lorentz factor (Table 1 of Zhang \& Mészáros 2002c). Thus any model may be adjusted to interpret the Amati relation given an input $E_{\text {iso }}-\Gamma$ relation. For example, in the internal shock model, the Amati relation could be reproduced if $\Gamma$ is insensitive to $E_{\text {iso }}$ (Zhang \& Mészáros 2002c). For photosphere-dominated prompt emission models, one needs a different $E_{i s o}-\Gamma$ 

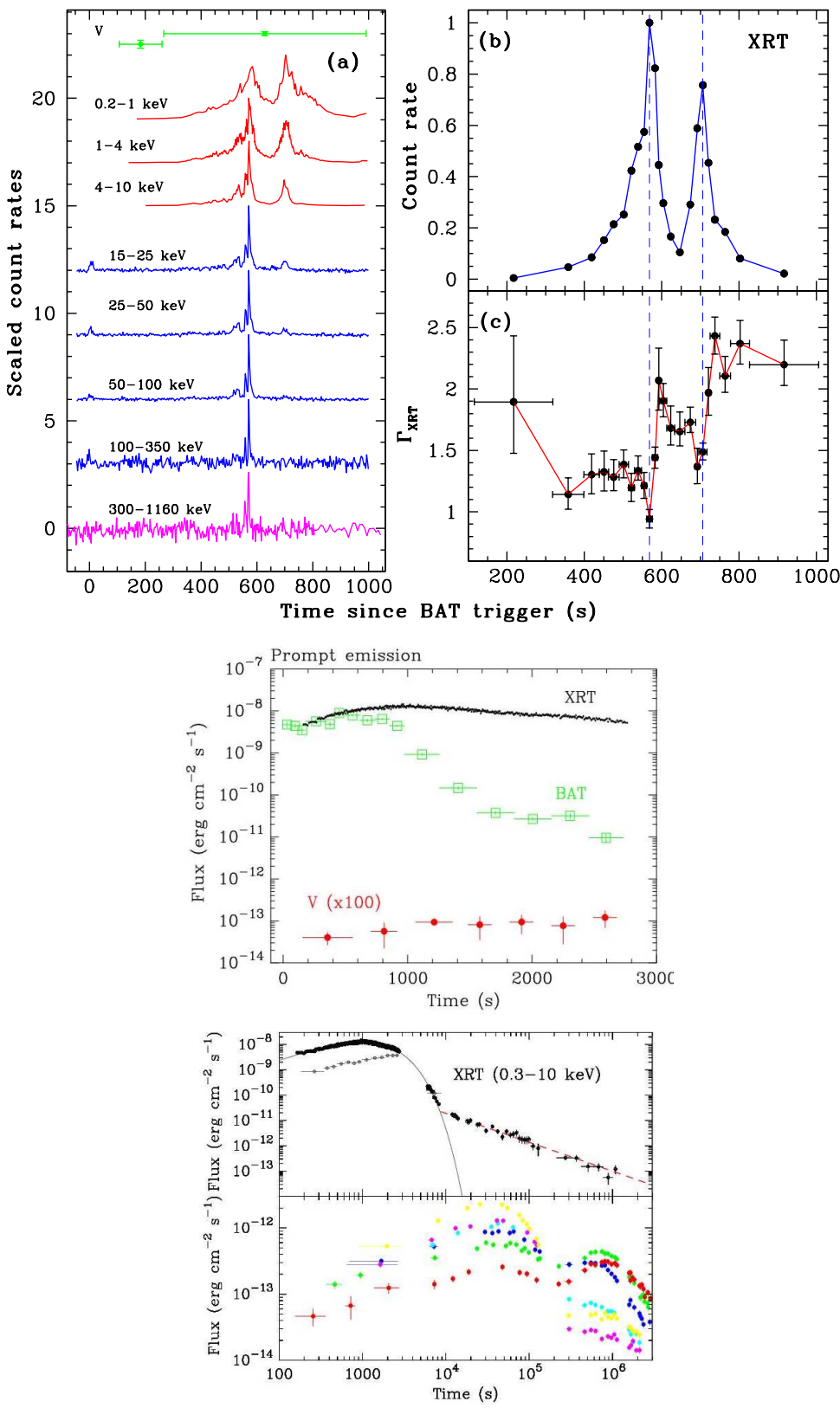

Fig. 7 Panchromatic observations the GRB prompt emission phase. Upper: GRB 060124 (Romano et al. 2006b); Lower: GRB 060218 (Campana et al. 2006a).

relation (or effectively $\theta_{j}-\Gamma$ relation if a $\theta_{j}-\Gamma$ relation is established) to interpret the Amati-relation, and such a correlation was regarded as more natural (Rees \& Mészáros 2005; Thompson et al. 2006). A similar argument was raised within a wind-deceleration model (Thompson 2006). On the other hand, if one assumes a standard emission unit in 
the comoving frame, the Amati-relation could be reproduced by the viewing angle effect for some types of jet configurations (e.g. Yamazaki et al. 2004a; Eichler \& Levinson 2004; Toma et al. 2005).

- Firmani relation: Firmani et al. (2006a) discovered a tight correlation with prompt emission data only, which reads $L_{p, i s o} \propto E_{p}^{3 / 2} T_{0,45}^{-1 / 2}$, where $T_{0,45}$ is the time of the enhanced burst emission. There has been no proposed interpretation of this relation.

- Frail, Ghirlanda, Liang-Zhang \& Willingale relations: For completeness, I repeat here the four empirical relations involving afterglow temporal breaks discussed in $\S 3.3$, but focusing on their interpretations. The Frail relation suggests a standard energy reservoir, a hypothesis which is not confirmed by the Swift data. The tight Ghirlanda and Liang-Zhang relations connect prompt emission properties $E_{p}$ and $E_{\gamma, \text { iso }}$ with the afterglow properties $\left(t_{j}\right.$ or $\left.t_{b}\right)$. It has been claimed that these relations could be understood within the ad hoc annular jet model with the assumption of a standard comoving radiation unit (e.g. Levinson \& Eichler 2005; Eichler \& Levinson 2006). The photosphere model (Thompson et al. 2006) may give a more physical interpretation. On the other hand, Swift XRT data now do not support the Ghirlanda relation (Sato et al. 2007; Willingale et al. 2006), which renders efforts to interpret the relation invoking a jet break not very meaningful. If one discards the jet framework, the rolling electron spectral break hypothesis (Zhang 2007, see $\S 3.3$ for more discussion) may be a possibility to interpret the Liang-Zhang relation. The close relationship between the Liang-Zhang relation and the Willingale relation seems to lend support to this suggestion. More data are needed to test the prediction of this scenario (§3.3).

Besides the above global properties, some Swift observations of GRBs have shed new light on the prompt emission mechanisms. In the following I'll list various pieces of (sometimes controversial) inference about GRB prompt emission drawn in the Swift era.

- Ryde (2005) argues that the so-called Band-function of GRB spectrum can be actually decomposed into the combination of a thermal and a non-thermal component. This model was further enriched by more physical models involving photosphere thermal emission and Compton dissipation above it (Ryde et al. 2006; Thompson et al. 2006). Time dependent modeling with latest Swift data is on-going (Ryde et al. 2007).

- Using some general observational constraints (but assuming shock dissipation and synchrotron/IC radiation mechanisms), Kumar et al. (2006) constrained the emission properties of two bursts (GRBs 050126 and 050219A) that display smooth and single-peaked gamma-ray lightcurves. The conclusion is that the radiation site is close to the deceleration radius (contrary to the closer-in photosphere radius derived from other arguments as discussed above), and that the radiation mechanism is synchrotron self-Compton. Kumar et al. (2007) used a larger sample to reach a similar conclusion about the large emission radius, and pointed out that neither internal shocks nor external shocks seem to interpret the data satisfactorily. A large emission radius is also independently estimated by Lyutikov (2006a) using the duration of the steep-decay GRB tails within the framework of curvature effect interpretation and by assuming a standard jet opening angle.

- A traditional problem of the synchrotron emission model of GRB prompt emission is the "fast-cooling" problem (Ghisellini et al. 2000). For standard parameters, the cooling frequency is much lower than the $100 \mathrm{keV}$ range, so that the predicted low-energy photon index is $-3 / 2$, steeper than that of most bursts (but is satisfied in some bursts, e.g. GRB 060124, Romano et al. 2006b). Using some general observational constraints, Pe'er \& Zhang (2006) derived the parameter sets of the internal shock synchrotron radiation model. They argued that the data could be reproduced if one assumes that the post-shock magnetic fields decay in a length scale much shorter than the comoving width, about $10^{5}-10^{6}$ skin 
depths. By introducing a synchrotron self-absorption break, the model can interpret the broad-band data of GRB 050820A. The suggestion may lead to a slow-cooling synchrotron model for prompt emission, which is consistent with the broad-band data of GRB 061121 (Page et al. 2006b). A similar suggestion has been proposed for afterglows (Rossi \& Rees 2003). The hypothesis is probably consistent with the ongoing numerical simulations of relativistic collisionless shocks (J. Arons 2006, personal communication).

- The broad-band data of super-long GRB 060218 (Campana et al. 2006a) during the prompt emission phase allow detailed modeling of GRB prompt emission for the first time. However, so far no model can successfully interpret the whole data set. The faint early UVOT observation severely constrains that the emission mechanism is likely not synchrotron (the extrapolation of the observed emission according to the synchrotron model predicts a much higher flux, Dai et al. 2006b). Ghisellini et al (2007) invokes synchrotron self-absorption to accommodate the optical flux deficit. The presence of a thermal X-ray component (probably due to shock breakout, Campana et al. 2006a, but see Li 2007) provides an extra source for inverse Compton emission (e.g. Dai et al. 2006b). A bulk Compton scattering model has been also proposed (Wang et al. 2006a), which suggests that the radiation mechanism of this (and probably also other) LL-GRBs may be different from that of canonical GRBs. On the other hand, the compliance of both Amati and Norris relations of this burst (Amati et al. 2006; Liang et al. 2006b) seem to suggest that its radiation physics should not be distinctly different from that of canonical GRBs. The hitherto most detailed prompt emission data of GRB 060218 seem to defy interpretation and to greatly challenge our basic understanding about the GRB radiation mechanism.

\subsection{Radiative efficiency}

One interesting question is the GRB radiative efficiency, which is defined as $\eta=E_{\gamma} /\left(E_{\gamma}+E_{K}\right)$, where $E_{\gamma}$ and $E_{K}$ are the isotropic gamma-ray energy and kinetic energy of the afterglow, respectively. The reason why $\eta$ is important to understand the explosion mechanism is that it is related to the energy dissipation mechanism of the prompt emission, which is not identified. The standard picture is internal shock dissipation, which typically predicts several percent radiative efficiency (Kumar 1999; Panaitescu et al. 1999, cf. Beloborodov 2000, Kobayashi \& Sari 2001). Other mechanisms (e.g. magnetic dissipation) may have higher efficiencies although a detailed prediction is not available. It is of great interest to estimate $\eta$ from the data, which can potentially shed light onto the unknown energy dissipation process.

In order to estimate $\eta$, reliable measurements of both $E_{\gamma}$ and $E_{K}$ are needed. While $E_{\gamma}$ could be directly measured from the gamma-ray fluence if the GRB redshift is known, measurement of $E_{K}$ is not trivial, as it requires detailed afterglow modeling. In the pre-Swift era, attempts to estimate $E_{K}$ and $\eta$ using late time afterglow data have been made (e.g. Panaitescu \& Kumar 2001, 2002; Freedman \& Waxman 2001; Berger et al. 2003b; Lloyd-Ronning \& Zhang 2004). The jet sideways expansion effect (Rhoads 1999; Sari et al. 1999; Huang et al. 2000) may somewhat affect the estimates of the efficiency (Zhao \& Bai 2006). The presence of an early shallow decay phase in Swift XRT afterglows suggest that $E_{K}$ likely increases with time. The $\eta$ values measured using the late time data are therefore no longer reliable. For a constant energy fireball, ideally early afterglows may be used to study the radiative loss of the fireball. However the shallow decay phase due to energy injection smears the possible signature and makes such a diagnosis difficult.

A systematic analysis of GRB radiative efficiencies using the first-hand Swift data has been carried out by Zhang et al. (2007b). Similar analyses using second-hand data for smaller samples of bursts were carried out by Fan \& Piran (2006a) and Granot et al. (2006). The conclusions 
emerging from these studies suggest that in most cases the efficiency is very high (e.g. > 90\%) if $E_{K}$ right after the burst is adopted. However, using $E_{K}$ at a later time when the injection phase is over one typically gets $\eta \sim$ several percent. The nature of the shallow decay phase is therefore essential to understand the efficiency. For example, if the shallow decay phase is due to continuous energy injection, the GRB radiative efficiency must be very high - causing problems to the internal shock model. If, however, the shallow decay is simply due to the delay of energy transfer into the forward shock (Kobayashi \& Zhang 2007), the GRB radiative efficiency is just the right one expected from the internal shock model. The investigation of Zhang et al. (2007b) also suggests that XRFs may not be intrinsically less efficient GRBs, in contrast to the pre-Swift expectation (Soderberg et al. 2004; Lloyd-Ronning \& Zhang 2004). Also as far as the radiative efficiency is concerned, there is no fundamental difference between Type I and Type II GRBs (see also Bloom et al. 2006a and Lee et al. 2005). This suggests that both types of GRBs share the same radiation physics.

\section{COSMOLOGICAL SETTING}

GRBs are cosmological events. The close connection between Type II GRBs with deaths of massive stars make GRBs potential tracers of star forming and probably metallicity history of the Universe. In view that the history of the Universe during the so-called "dark age" (from cosmic background radiation at $z \sim 1100$ to the epoch when first quasars were formed around $z \sim 7$ ) is still poorly known (Loeb \& Barkana 2001 for a review), GRBs, as bright beacons in the deep Universe, would be the unique tool to illuminate the dark Universe and allow us to unveil the re-ionization history of the Universe. There are several reasons to believe that high- $z$ GRBs exist and are detectable. First, due to a favorable $k$-correction factor and the time-dilation effect, theoretically high- $z$ GRBs are not much dimmer than their nearby sisters for both prompt gamma-ray emission and afterglow in the infrared and radio wavelengths (Lamb \& Reichart 2000; Ciardi \& Loeb 2000; Gou et al. 2004; Ioka \& Mészáros 2005). In fact, the GRB redshift record holder GRB 050904 (Cusumano et al. 2006a; Haislip et al. 2006; Kawai et al. 2006; Frail et al. 2006) at $z=6.295$ has very bright prompt gamma-ray emission and early infrared and radio afterglows. Second, based on several empirical standard candles (e.g. Fenimore \& RamirezRuiz 2000; Norris et al. 2000; Amati et al. 2002) one could derive the "pseudo"-redshifts of a large sample of GRBs. The results suggest that over $10 \%$ of GRBs are at $z>6$. This is also consistent with the theoretical prediction of the GRB rate assuming GRBs tracing the cosmic star formation history (Bromm \& Loeb 2002, 2006). Third, numerical simulations suggest that first generation stars form at around $z \sim 20$ (Bromm et al. 1999; Abel et al. 2002), which is generally also consistent with the conclusion drawn from the cosmic microwave background data collected by WMAP (Bennett et al. 2003 Spergel et al. 2006). Finally, a high fraction of high- $z$ bursts is also inferred from redshift distribution of Swift bursts (Jakobsson et al. 2006a). It is highly expected that GRBs would break the current redshift record held by faint galaxies, which would then bring unprecedented information about the reionization history of the early Universe. The first two year of Swift observations have detected at least four bursts with $z>5$ : GRB 050814 at $z=5.3$ (Jakobsson et al. 2006b), GRB 050904 at $z=6.29$ (Cusumano et al. 2006a; Haislip et al. 2006; Kawai et al. 2006), GRB 060522 at $z=5.11$ (Cenko et al. 2006), and GRB 060927 at $z=5.6$ (Fynbo et al. 2006b). The lower rate than predicted is very likely due to the challenge of promptly performing IR spectroscopic observations of the high- $z$ bursts. The low UVOT detection rate of Swift GRBs (Roming et al. 2006a) could be partially due to a good fraction of high- $z$ GRBs. In fact, based on prompt emission data, some high- $z$ GRB candidates have been suggested (e.g. GRB 050717, Krimm et al. 2006b).

The study of high- $z$ GRBs reveals interesting features. GRB 050505 (Hurkett et al. 2006) at $z=4.275$ has a host galaxy with a damped Lyman-alpha system with the highest column 
density (Berger et al. 2005c). High resolution spectroscopy reveals fine-structure transition features which can be used to infer gas densities and diffuse radiative conditions of the host galaxy (Chen et al. 2005). The study of the GRB redshift holder 050904 (Cusumano et al. 2006a; Haislip et al. 2006; Kawai et al. 2006; Watson et al. 2006) is even more fruitful: A detailed spectroscopic study (Totani et al. 2006) suggests that the Universe is already largely ionized at $z=6.3$. Afterglow observations (Frail et al. 2006) and modeling (Gou et al. 2006) reveal a relative high density circumburst medium around the burst. The detection of a bright optical flare similar to GRB 990123 (Boër et al. 2006) suggests a possible bright reverse shock emission component (Wei et al. 2006; Gou et al. 2006). The most erratic flaring activity in X-rays (Cusumano et al. 2006a,c) suggests a super-long active central engine (e.g. Zou et al. 2006). A speculation is that this might be related to a more massive (or probably more rapidly-rotating) progenitor star.

An interesting question is whether GRB properties evolve with redshift. The possibility has been raised in the literature based on various different arguments (e.g. Lloyd-Ronning et al. 2002; Wei \& Gao 2003; Donaghy et al. 2004; Firmani et al. 2004; Salvaterra \& Chincarini 2006). On the other hand, observational selection effects (e.g. only bright GRBs are detectable at high redshifts), which are difficult to address, tend to mimic an apparent evolutionary pattern, rendering a robust claim of evolutionary effect difficult. A statistical study of GRBs with known redshift (Liang et al. 2006c) suggests that the observed luminosity and redshift distributions could be well reproduced without introducing evolutionary effects. More data are needed to draw firmer conclusions. From the theoretical point view, first generation stars tend to be massive due to their low metallicity (Abel et al. 2002). If these stars also produce GRBs, the bursts may be more energetic. The deaths of these stars, however, quickly contaminate the interstellar medium, so that the next generation stars may not be very different from the stars seen now. The evolutionary pattern, if any, may be more complicated than a simple power law dependence on $(1+z)$.

Another interesting question is whether (Type II) GRBs trace the cosmic star forming history only (e.g. Totani 1997). Tentative evidence that metallicity is another important factor to make a GRB has been collected (e.g. Ramirez-Ruiz et al. 2002b; Prochaska et al. 2004; Stanek et al. 2006; Modjaz et al. 2007, cf. Campana et al. 2007). This factor is currently not included in most GRB population studies (e.g. Perna et al. 2003; Lloyd-Ronning et al. 2004; Zhang et al. 2004a; Lamb et al. 2005a; X. Dai \& Zhang 2005; Guetta et al. 2005; Lin et al. 2005; Xu et al. 2005b; Liang et al. 2006c, etc). It is interesting to explore how to incorporate the metallicity factor in a quantitative way and how different the results would be with the metallicity factor included.

The study of Type I GRBs within the cosmological context has just started (e.g. Nakar et al. 2006a; Guetta \& Piran 2006; Belczynski et al. 2006; Berger et al. 2006b). The data are consistent with there being a delay of Type I GRBs with respect to the star forming history of the Universe. Better understanding of the cosmological setting of Type I GRBs will be achieved in a few years when more data become available.

I'd like to finish this section by discussing an exciting but controversial field: the GRB cosmology. The cosmological setting of (Type II) GRBs suggests that they can be invaluable tools to measure the structure of the Universe if GRBs are standard candles. Since GRBs have higher redshifts than the Type Ia SNe, it is promising that GRBs would extend the measurement of the Universe to the high-redshift regime that Type Ia SNe cannot attain. The fundamental question is whether there exists a physically-understood, narrowly-clustered tight correlation that can serve as a standard candle. Most previously claimed GRB correlations have been listed in $\S 3.3$ and $\S 3.4$. Earlier attempts to build GRB Hubble diagrams (e.g. Schaefer 2003; Bloom et al. 2003) have failed to put meaningful constraints on the cosmological parameters, 
since the correlations that were used have very large scatter. It was after the discovery of the tight Ghirlanda correlation (Ghirlanda et al. 2004b) when the GRB cosmology started to make progress (Dai et al. 2004; Ghirlanda et al. 2004c; Xu et al. 2005a; Firmani et al. 2005; Xu 2005). The approach was however criticized by Friedman \& Bloom (2005) who pointed out several uncertainties inherited in the Ghirlanda relation. Liang \& Zhang (2005) discarded the jet model and proposed the model-independent $E_{\text {iso }}-E_{p}-t_{b}$ correlation, which is tight enough for the cosmological purpose. Lately, Firmani et al. (2006a) discovered a tight correlation using prompt emission data only, and use it to perform a cosmological study (Firmani et al. 2006b). By combining the GRB standard candles with the Type Ia SNe data, useful constraints can be placed on a list of cosmological models. The results are generally consistent with the concordance cosmology revealed by WMAP, while the high- $z$ nature of GRBs allows the data to start to put useful constraints on dynamical dark energy models (Firmani et al. 2005; Wang \& Dai 2006; Nava et al. 2006; Qin et al. 2006b; Mosquera Cuesta et al. 2006; Su et al. 2006; Li et al. 2006; Schaefer 2007).

There are two fundamental difficulties in the GRB cosmology. The first one is the calibration problem. Since most GRBs are at high-redshifts where cosmological effects are important, and since nearby GRBs may belong to a different population, it is essentially impossible to calibrate a GRB standard candle using a low- $z$ GRB sample, as has been done for Type Ia SNe. Without a calibrated candle, there is a circularity problem by using a candle determined from one cosmology to constrain cosmological parameters. The problem could be partially solved by collecting a sample of GRBs within a redshift bin (e.g. Lamb et al. 2005b; Ghirlanda et al. 2006; Liang \& Zhang 2006b). In particular, Liang \& Zhang (2006b) showed that one can well calibrate the power law indices of various standard candle correlations with the method. The coefficient cannot be calibrated, but may be "marginalized" within a range of cosmologies. The required redshift bin is not too narrow, say, $\Delta z \sim 0.3$, so that it may be possible to calibrate the GRB candles in the near future when the sample grows to a large enough size. The second, more fundamental difficulty is to identify a physically-based standard candle. As have been discussed in $\S 3.3$ and $\S 3.4$, the physical origins of Ghirlanda, Liang-Zhang and Firmani relations are still not identified. More frustratingly, the Ghirlanda relation is not confirmed by the Swift XRT data (Sato et al. 2007; Willingale et al. 2006), suggesting that the relation is not attached to the jet framework. In the pre-Swift era, it has been assumed that the relations are generally valid (e.g. Firmani et al. 2005; Xu et al. 2005a) and simulations were made to see how large a sample is required in order to achieve a certain constraint on cosmology. The observations by Swift suggest that when detecting a burst, the very first thing to do is to check whether the previously proposed standard candles are still satisfied. The growing outliers to the Ghirlanda relation seem to suggest that one may need to discard it as a useful cosmological tool. The deficiency of late optical data does not allow a clear test to the Liang-Zhang relation at the moment. The Firmani relation makes use of prompt emission data only and is easier to test. It is probably by far the best GRB standard candle. However, the physical origin of the correlation is not understood yet.

\section{OUTSTANDING PROBLEMS}

Although most have been discussed before, it is informative to summarize the outstanding GRB problems as of late 2006 .

- GRB classifications and progenitor systems: Are there only two major types of GRBs or there is a third major category with a distinct progenitor system? Are NS-NS mergers distinct from BH-NS mergers (or other mergers)? Within the Type II (collapsar-related) 
GRBs, are LL-GRBs distinctly different from HL-GRBs? What is the very nature of XRFs? Why there are two apparent universal tracks for intrinsic optical afterglows?

- GRB central engine: How are relativistic jets launched? For distinctly different progenitor systems, how could the central engines be so similar? In particular, how could a central engine be restarted to sustain erratic long-term activities to power X-ray flares for both collapsar-type (Type II) and merger-type (Type I) GRBs? Does the central engine also inject energy steadily for a long time? If so what is the observational evidence? It is worth commenting that the latest analysis of Swift data starts to reveal smoothly decaying components that are not interpretable within the standard external shock scenarios (Willingale et al. 2006; Zhang et al. 2007c). Another comment is that magnetic fields likely play an important role at the central engine (e.g. Usov 1992; Thompson 1994; Mészáros \& Rees 1997b), but due to intrinsic complications, MHD is usually not incorporated in the central engine models, except for several fruitful first attempts (e.g. Proga et al. 2003; Mizuno et al. 2004a,b).

- Composition of the GRB outflow: Are GRB outflows matter dominated or Poyntingflux dominated? What is the evidence for/against either possibility? A comment here is that the matter-dominated model has been regarded as standard - as long as the data can be accommodated within the matter-dominated model, the Poynting-flux-dominated model is not needed. In reality, magnetic fields should play an important role at the central engine, and some tentative evidence of a highly magnetized flow (e.g. bold gamma-ray polarization, Coburn \& Bogg 2003, cf. Rutledge \& Fox 2004; Kalemci et al. 2007, the requirement a higher $\epsilon_{B}$ in the reverse shock, Fan et al. 2002; Zhang et al. 2003; Kumar \& Panaitescu 2003; Fan et al. 2005c; Wei et al. 2006, as well as the energetics argument for X-ray flares, Fan et al. 2005d) has been collected. The GRB outflow should be at least hybrid (e.g. with a moderate $\sigma$ parameter). Studies (e.g. Fan et al. 2004a; Zhang \& Kobayashi 2005; Fan et al. $2004 \mathrm{~b}$ ) show that the differences with respect to the pure hydrodynamical models are not prominent for $\sigma<1$. In high- $\sigma$ regime the dynamical behaviors of the outflow are still not fully understood (see Zhang \& Kobayashi 2005; Lyutikov 2006b for preliminary discussions), and detailed MHD simulations are needed. Due to intrinsic degeneracy of model predictions (e.g. the reverse shock emission is not significant for both low- $\sigma$ and high- $\sigma$ flows, e.g. Zhang \& Kobayashi 2005), a direct diagnosis of GRB composition from the data is not an easy task.

- GRB prompt emission mechanism and site: Are prompt gamma-rays produced in internal shocks, at the photospheres, or in magnetic reconnection regions? Is the emission site "closer-in" (near photosphere) or "further-out" (near deceleration radius)? Is the thermal component important in the spectrum? (It is noted that a thermal component may be also required to fit some of the X-ray flare spectrum, e.g. Grupe et al. 2006b.) What is the non-thermal mechanism - synchrotron or Comptonization? Related questions would be what powers high energy emission (leptonic vs. hadronic) and whether GRBs are emitters of cosmic rays and high energy neutrinos. These topics will be discussed in $\S 6$.

- GRB jet configuration: Are GRBs collimated at all (this question arises after Swift detected a good list of GRBs without showing a break in X-rays several months after the triggers, Willingale et al. 2006; Burrows 2006)? If so, what is the collimation angle (this question is raised since some temporal breaks are not achromatic, so that one could not always simply use afterglow breaks to estimate the jet opening angles)? Are jets structured (maybe needed to interpret some bursts such as GRB 060124, Romano et al. 2006b, and GRB 061007, Schady et al. 2006b)? What conclusion could one draw regarding the energetics of GRBs and their statistical properties (the simple picture of standard energy reservoir no longer applies). 
- Properties and origins of the afterglows: What are the origins of the distinct afterglow components (especially the shallow decay component)? How much can the external shock model explain? What is the role of the central engine and the internal dissipation regions? What is the nature of temporal breaks, especially the chromatic ones? Swift observations seem to suggest that what we call "afterglows" actually include both the traditional external component and some other components unrelated to the external shocks. X-ray flares are a good example of a distinct (late internal dissipation) origin. Even some smoothly decaying components may be also related to the central engine or the internal dissipation regions (e.g. Fan et al. 2006; Zhang et al. 2006c). The phenomenological two-component modeling of Willingale et al (2006) seems to be able to fit most of the X-ray afterglows. One is driven to consider the physical origins of the fitting. The most puzzling question is the nature of the afterglow temporal breaks, especially those that are not achromatic. Extensive data mining and sorting are needed to see whether some ad hoc scenarios (e.g. Zhang 2007) are indeed needed to understand the breaks.

- Properties of GRB environment: What is the immediate environment of GRBs? Very early data collected by Swift and other ground-based telescopes have allowed a diagnosis of the immediate environment of GRBs. The data suggest that the GRB immediate environment is a constant density medium rather than a stratified stellar wind (Zhang et al. 2006, 2007b; Molinari et al. 2006; Still et al. 2005; Blustin et al. 2006). It then more seriously raises the question why a Type II GRB preferentially lies in a constant density medium. Other questions include: Is the ambient density of Type I GRBs lower than that of Type II GRBs? Is the ambient medium clumpy (e.g. Dermer 2006)? Is the ambient medium magnetized (e.g. Li \& Waxman 2006)? Is there an evolution of medium density with redshift (Gou et al. 2004)? Are the dust and extinction properties of GRB host galaxies significantly different from those of Milky Way or SMC/LMC (Chen et al. 2006)?

- Properties of GRB shocks: Are the electrons accelerated to a power law distribution? Is the electron power law index universal or unpredictable (The data seem to suggest no universality of $p$ among GRBs, e.g. Shen et al. 2006, cf. Wu et al. 2004; see also Dai \& Cheng 2001 for discussion of the $p<2$ afterglows)? What define the shock microphysics parameters (e.g. $\epsilon_{e}, \epsilon_{B}$, etc)? Are there correlations between these parameters (e.g. Medvedev 2006)? Do microphysics parameters evolve with time (e.g. Yost et al. 2003; Ioka et al. 2006; Panaitescu et al. 2006b; Fan \& Piran 2006a)? Numerical (particle-in-cell) simulations and analytical studies have started to answer the fundamental questions about particle acceleration and magnetic field generation (e.g. Medvedev \& Loeb 1999; Nishikawa et al. 2003, 2005; Liang \& Nishimura 2004; Hededal \& Nishikawa 2005; Spitkovsky 2005; Kato 2005; Milosavljevic \& Nakar 2006a,b).

\section{OUTLOOK}

Although unprecedented information has been collected for GRBs, there are yet more observational channels that are deemed to be important to study GRBs, but are so far sparsely covered. These include the electromagnetic spectrum above $10 \mathrm{~s}$ of $\mathrm{MeV}$, and non-electromagnetic signals such as high energy neutrinos and gravitational waves. These observations are widely expected to be made in the near future.

\subsection{GRB science with GLAST}

The launch of GLAST (Gehrels \& Michelson 1999) in late 2007 will open a new era for GRB studies. The Large Area Telescope (LAT) on board has a wide energy range from $<20 \mathrm{MeV}$ to $>300 \mathrm{GeV}$. A dedicated GLAST Burst Monitor (GBM) with energy coverage from $\sim 8$ 
$\mathrm{keV}$ to $\sim 30 \mathrm{MeV}$ will promptly localize GRBs and perform spectral analysis of the bursts. Complemented by other space- and ground- based high energy photon detectors (e.g. Milagro, Dingus et al. 2004; VERITAS, Horan et al. 2005; AGILE, Pittori \& Tavani 2005; HESS, Hinton 2004; MAGIC, Lorenz 2004; CANGAROO-III, Kubo et al. 2004), GLAST will unveil the last spectral window of GRB observations. With the overlapping operations of both Swift and GLAST, GRBs will be studied with a full spectral and temporal coverage for the first time.

Observationally, tentative evidence of distinct high-energy components has been collected in the past. Hurley et al. (1994) detected long-lasting high energy emission from GRB 940217, which extended 90 minutes after the trigger and included one $18 \mathrm{GeV}$ photon. Gonzalez et al. (2003) reported the existence of a distinct high energy component in GRB 941017 which is spectrally and temporally decoupled from the conventional sub-MeV component. Atkins et al. $(2000,2003)$ suggested evidence for TeV emission from GRB 970417A by reporting the observation by Milagrito (the prototype detector of Milagro) that reveals an excess of events coincident in time and space with the burst.

On the theoretical side, the fireball model is not short of mechanisms to produce these high energy photons. In fact, one could list over a dozen of mechanisms to produce high energy photons from a relativistic fireball. The challenge is how to identify the correct mechanism at work. This is also related to the unknown GRB composition as well as the origin(s) of the prompt emission and afterglow. The following is an unexhausted list, according to the increasing distance from the GRB central engine of the high energy emission site.

- During fireball acceleration, protons and neutrons may be decoupled if the fireball entropy is high enough (Derishev et al. 1999; Bahcall \& Mészáros 2000). Inelastic collisions between neutron and proton streams would produce neutrinos and $\mathrm{GeV}$ photons (Bahcall \& Mészáros 2000). For nearby ( $z \sim 0.1$ ) Type I, neutron-loaded GRBs of merger origin, GLAST may be able to detect prompt $100 \mathrm{MeV}$ and $100 \mathrm{GeV}$ photon signatures from this process (Razzaque \& Mészáros 2006a). For neutron-rich ejecta, beta decay of the free neutrons would also give unique temporal and spectral signatures that may be used to diagnose the presence of free neutrons (Razzaque \& Mészáros 2006b).

- In internal shocks, if the sub-MeV emission that triggers gamma-ray detectors is due to synchrotron emission, then a synchrotron self-Compton (SSC) component naturally extends to high energies. High energy photons are likely attenuated with low energy photons to produce pairs, whose secondary emission also contribute to the observed spectrum (e.g. Mészáros et al. 1994; Pilla \& Loeb 1998; Razzaque et al. 2004a; Pe'er \& Waxman 2004a, 2005; Takagi \& Kobayashi 2005; Pe'er et al. 2005, 2006a).

- In internal shocks, protons are also accelerated. Their synchrotron emission or photonmeson interaction would also lead to high energy photon emissions. Assuming optimistic parameters, these emission signatures may be detectable (e.g. Totani 1998; Bhattacharjee $\&$ Gupta 2003). However, in a large parameter space (e.g. $\epsilon_{e}$ not extremely low), the proton radiation components and the secondary emission of the leptons produced in photo-meson interactions are not as significant as the electron SSC process and therefore not detectable (Fragile et al. 2004; Razzaque \& Zhang 2007; Gupta \& Zhang 2007b).

- In the external reverse shock, SSC would produce high energy photons in the GeV range (e.g. Mészáros et al. 2003; Wang et al. 2001a; Granot \& Guetta 2003, cf. Kobayashi et al. 2007).

- In the external forward shock, SSC at early times also produces significant GeV emission that is detectable by GLAST (e.g. Mészáros \& Rees 1994; Dermer et al. 2000; Zhang \& Mészáros 2001b, for more general discussion of SSC process in the external forward shock see Wei \& Lu 1998, 2000a; Panaitescu \& Kumar 2000; Sari \& Esin 2001; Wu et al 2005b). In particular, Zhang \& Mészáros (2001b) showed that for the shock parameter regime 
commonly inferred from the broadband afterglow fits, the SSC component is prominent and detectable by GLAST for GRBs at $z \sim 1$. Due to the slow crossing of the SSC peak energy in the GLAST band, GLAST would be able to detect these extended GeV emissions for hours after the trigger. It is worth commenting that the calculation of Zhang \& Mészáros (2001b) was made by assuming a constant energy in the fireball. The shallow decay phase revealed by Swift XRT may suggest substantial energy injection in the early phase (§3.1.3). If this is the case, the SSC signature may be weakened. The presence of X-ray flares would also cool electrons in the external shock (Wang et al. 2006b; Gou et al. 2006). This suggests a less optimistic prediction of the expected $\mathrm{GeV}$ signals due to $\mathrm{SSC}$ in the forward shock region. This model was also used to interpret the distinct hard component in GRB 941017 (Pe'er \& Waxman 2004b).

- Photons from the forward and reverse shock regions could be inverse Compton scattered by electrons in the other regions. These cross IC processes are important high energy emission contributors (Wang et al. 2001a,b).

- The prompt sub-MeV photon bath may overlap the external shock region (both reverse shock, Beloborodov 2005; Fan et al. 2005e, and forward shock, Fan et al. 2005e) if the burst duration is long enough. The electrons in the shocked region would cool by scattering these prompt gamma-rays and produce high energy photons (Beloborodov 2005). The effect is especially important in a wind medium where the deceleration radius is small (Fan et al. 2005e).

- Protons in the external shock region would produce high energy photons through synchrotron emission and photo-meson interaction (Böttcher \& Dermer 1998). The parameter space for this component to dominate is small (i.e. $\epsilon_{e} \ll \epsilon_{B}$, Zhang \& Mészáros 2001b), and is not the preferred parameter space derived from the broad-band afterglow fits.

- Photons from X-ray flares and probably unobserved UV flares would be upscattered by the external shock electrons to produce GeV-TeV photons (Wang et al. 2006b; Fan \& Piran $2006 \mathrm{~b})$.

- SSC within the X-ray flares would produce high energy photons (Wang et al. 2006b).

- If additional soft photons are available from the GRB progenitor, external IC processes would boost soft photons to high energies. For example, such a process may happen in GRBs associated with SNe (such as GRB 060218) from which thermal photons due to the putative SN shock breakout are reprocessed and boosted in energy (Wang \& Mészáros 2006).

- TeV photons escaping from GRB fireballs would be attenuated by intergalactic infrared background and produce pairs, if the GRB source is not too close to earth (say $z<0.5$ ). These pairs would upscatter the cosmic microwave background and produce GeV photons, which would be detectable by GLAST if the IGM magnetic field is weak enough (to avoid significant deflection of pairs before the interaction with CMB happens). Such a process would give rise to a delayed high energy emission following the GRB prompt emission (Plaga 1995; Cheng \& Cheng 1996; Dai \& Lu 2002a; Wang et al. 2004; Razzaque et al. 2004a).

- If a GRB occurred in the past in our galaxy, it is expected that significant $\mathrm{GeV}-\mathrm{TeV}$ emissions occur from the GRB remnant (Ioka et al. 2004).

- For both prompt and delayed high energy emissions, even if they are not directly detectable, they could contribute to the gamma-ray diffuse background. A more careful investigation (Casanova et al. 2007) suggests that for most optimistic parameters, GRBs are not the dominant contributor to the diffuse gamma-ray background.

Although all the above possibilities have been suggested, it is now high time to perform a more systematic study of the relative importance of various mechanisms. Since early afterglow data have been extensively retrieved by Swift, one can perform more realistic calculations with 
the constraints posed by low energy prompt emission and afterglow data. Such predictions, when compared with future GLAST data, would give strong constraints on both low energy and high energy models, narrow down and identify the physical processes happening in GRBs, and shed light on some outstanding problems listed in $\S 5$.

A prospect of GLAST observation is to constrain the bulk Lorentz factor of GRBs. This is an important unknown parameter of GRB fireball. Due to internal photon-photon productions, it is expected that there would be a (sharp) spectral cutoff in the prompt GRB spectrum, which has not been clearly detected. In the past, the highest energy photons have been used to constrain the lower limit of GRB fireballs (Woods \& Loeb 1995; Baring \& Harding 1997; Lithwick \& Sari 2001). With the detection of a clear spectral cutoff, by combining the variability data, one can give interesting estimates to the GRB bulk Lorentz factors (Baring 2006). These results could be compared with the Lorentz factor lower limit derived from the early X-ray afterglow data (Zhang et al. 2006) and sometimes independent measurements using the early optical afterglow data (e.g. Molinari et al. 2006). If the $\Gamma$ measurements of a good sample of GRBs become available, statistical work could be carried out to check how $\Gamma$ is correlated with $E_{i s o}, L_{i s o}, E_{p}$, etc. These correlations hold the key to identify the correct prompt gamma-ray emission model (§3.4). It is worth commenting that gamma-rays become transparent from the fireball again at even higher energies (e.g. PeV), and the opaque window becomes narrower with a higher bulk Lorentz factor (Razzaque et al. 2004a).

Swift observations led to many surprises. Is it possible to make some reasonable predictions for GLAST? The chance to make such predictions in the pre-GLAST era is better than that in the pre-Swift era, mainly because we already have detailed information about both the prompt emission and afterglow in the "low energy" regime. The following is a list of bold, rough predictions for what GLAST would detect for GRBs.

- It is almost guaranteed to detect prompt emission in the GLAST band, with a possible spectral cut-off. The exact location of the cut-off depends on the properties of the burst. Generally Type-II GRBs would have higher fluences and Type-I GRBs would have lower fluences, mainly because their low energy counterparts are such. Type-I GRBs may have a higher cut-off energy than Type-II GRBs (a harder spectrum and probably a higher $\Gamma$ ), but this is not guaranteed. XRFs may not have significant high energy emission.

- High energy emission typically lasts longer than the sub-MeV prompt emission (due to many possible reasons listed above). The spectrum would have a temporal evolution. Harder photons tend to be detected at later times when the fireball becomes less compact for photons.

- At the low energy regime in the GLAST band, the prompt emission lightcurves would have narrower spikes than the the sub-MeV lightcurves, a general trend revealed by Swift panchromatic observation (e.g. Romano et al. 2006b; Page et al. 2006b). However, at higher energies when the putative IC component takes over, the lightcurves would be more smeared out with less sharp spikes due to the non-linear IC processes involved.

- It is possible that GLAST would detect bursts for thousand of seconds. The long-lasting emission may have a broad temporal bump with flares overlapping on top of it. The rising and falling indices of the flares would be less steep (again due to the non-linear IC processes), and the flare amplitudes would be smaller than those of X-ray flares.

More concrete predictions require more detailed study. As suggested by past experience, surprises and new challenges are also bound to merge in the GLAST era. 


\subsection{Other future observations}

GRB shocks are ideal sites to accelerate cosmic rays. It has been argued that they are a good candidate to generate ultra-high energy cosmic rays (UHECRs, Waxman 1995; Vietri 1995; Migrom \& Usov 1995; Vietri et al. 2003; Waxman 2004b). GRBs are also emitters of neutrinos of a wide range of energy. Neutrinos are one of the main agent to launch the relativistic jet from the central engine. MeV neutrinos from core collapses would escape. Unless the bursts are close enough, these $\mathrm{MeV}$ thermal neutrinos are however undetectable. Proton-neutron decoupling during the acceleration phase (Derishev et al. 1999) would produce multi-GeV neutrinos (Bahcall \& Mészáros 2000). pp interaction in internal shocks could produce $30 \mathrm{GeV}$ neutrinos (Paczyński \& Xu 1994). Within the collapsar scenario, $p \gamma$ and $p p$ interactions in internal shocks within the stellar envelope would give multi-TeV neutrinos, regardless of whether the jet would successfully penetrate through the envelope to make a successful GRB (Mészáros \& Waxman 2001; Razzaque et al. 2003, 2004b). In the internal shocks that produce observable GRB prompt emission, $p \gamma$ interactions typically produce $10^{14} \mathrm{eV}$ neutrinos (Waxman \& Bahcall 1997; Rachen \& Mészáros 1998). For LL-GRBs such as GRB 060218, this component typically emits neutrinos at higher energies (above $10^{17} \mathrm{eV}$ ), and is probably the most important GRB emission component in this energy range, thanks to the very high event rate of LL-GRBs (Gupta \& Zhang 2007a; Murase et al. 2006). X-ray flares of late internal shock origin should be also accompanied by neutrino emission (Murase \& Nagataki 2006). In the external shock region, GRBs produce neutrinos with even higher energies ( EeV, Waxman \& Bahcall 2000; Dai \& Lu 2001; Dermer 2002; Li et al. 2002). A generic upper limit of the extragalactic neutrino flux has been set up by Waxman \& Bahcall (1999, see also Bahcall \& Waxman 2001) using the observed UHECR flux. A list of $\mathrm{km}^{3}$ neutrino experiments, e.g. ICECUBE (Hill 2006), ANITA (Barwick et al. 2006), KM3Net (Katz 2006), Auger (Van Elewyck et al. 2006) are being built and are expected to detect these possible high energy neutrino signals from GRBs. The detections of high energy neutrinos from GRBs not only help to constrain GRB models, but are also valuable for studying neutrino physics (e.g. Li et al. 2005; Gonzalez-Garcia \& Halzen 2006). Several caveats need to be mentioned regarding GRBs as neutrino emitters. First, in order to maximize the predicted neutrino flux, usually a $p=2$ proton spectrum is assumed. Studies of prompt and afterglow emission suggests that $p$ is typically steeper than 2 for electrons. If protons also have $p>2$, the predicted neutrino flux would drop. Second, usually the neutrino spectrum for a burst with typical parameters is taken to estimate the diffuse neutrino flux. In principle, one needs to average over bursts with a wide range of distributions of luminosity and other parameters. Such an analysis (Gupta \& Zhang 2007a) suggests that the predicted diffuse background emission sensitively depends on some unknown parameters, especially the bulk Lorentz factor of GRBs. The predicted diffuse neutrino flux level is therefore rather uncertain. On the other hand, the detection (or tight upper limit) would present severe constraints on the bulk Lorentz factor distribution of GRBs. Finally, all the calculations have been performed under the assumption of the baryonic fireball model. If GRB outflows are Poynting-flux dominated, and if the prompt emission is due to magnetic reconnection rather than shock acceleration, GRBs are not important contributors to UHECRs and high energy neutrinos.

GRBs are also good candidate gravitational wave (GW) sources. The two leading progenitor candidates for GRBs, i.e. mergers of binary compact objects (Thorne 1987; Phinney 1991; Kochanek \& Piran 1993; Kobayashi \& Mészáros 2003) and stellar core-collapses (Rampp et al. 1998; van Putten 2001; Fryer et al. 2002; Kobayashi \& Mészáros 2003), have been suggested as potential GW sources. Fragmentation and subsequent accretion of a collapsing star (King et al. 2005; Piro \& Pfahl 2006) and acceleration of a GRB jet (Sago et al. 2004) would also excite GW of different wave forms. A coincidence between a GW burst and a GRB would greatly enhance the statistical significance of the GW signal, making detections easier (Finn 
et al. 1999). The GW frequencies of various phases (in-spiral, merger and ring-down) of both types of progenitor cover the $10-10^{3} \mathrm{~Hz}$ band which is relevant to several GW detectors, such as LIGO (Abramovici et al. 1992), VIRGO (Tournefier et al. 2005), GEO600 (Benno et al. 2003) and TAMA300 (Fujimoto et al. 2005). Due to the intrinsic faintness of the signals, only nearby sources (within $\sim 200 \mathrm{Mpc}$ for NS-NS and NS-BH mergers, and within $\sim 30 \mathrm{Mpc}$ for collapsars, Kobayashi \& Mészáros 2003) have strong enough signals to be detected by LIGO-II. Recent observations of short GRBs of merger origin suggest a higher event rate than estimated previously (Nakar et al. 2006a). This is encouraging for GW detections of GRBs.

\section{CONCLUSION}

Swift has greatly revolutionized our understanding of GRBs. Comparing with the status of the pre-Swift era (e.g. Zhang \& Mészáros 2004), we have learned a lot about GRB classification (e.g. the nature of "short" GRBs, §2), GRB physics (e.g. early afterglow properties, prompt emission site, etc, $\S 3$ ) and their cosmological setting (§4). However, new questions and challenges arise (e.g. §5). In particular, some pre-Swift pictures (e.g. the nature of afterglow breaks and the inference about GRB jet configuration and energetics) have to be modified or even abandoned. $\mathrm{X}$-ray flares open a new era of central engine study. Time is ripe to perform systematic data analyses to peer into the global properties of the bursts. While one can still gain knowledge from special individual events (such as GRB 060218 and GRB 060614), for most of the "normal" bursts, only global statistical properties can serve to improve our understanding of GRBs. Swift has collected and will keep collecting an unprecedented GRB sample for both prompt emission and afterglows. Systematical studies of this sample have just commenced (e.g. O'Brien et al. 2006b; Willingale et al. 2006; Zhang et al. 2007b,c; Chincarini et al. 2007; Butler \& Kocevski 2007).

The author thanks D. N. Burrows, G. Chincarini, X. Dai, Z. G. Dai, Y. Z. Fan, N. Gehrels, L. J. Gou, N. Gupta, Y. F. Huang, K. Ioka, S. Kobayashi, P. Kumar, E. W. Liang, T. Lu, P. Mészáros, E. Nakar, J. Norris, P. O’Brien, A. Panaitescu, A. Pe'er, R. Perna, Y. P. Qin, E. Ramirez-Ruiz, S. Razzaque, X. Y. Wang, D. M. Wei, R. Willingale, X. F. Wu, D. Xu, and R. Yamazaki for comments on the paper. This work is supported by NASA under grants NNG06GH62G and NNG05GB67G.

\section{References}

Abel, T., Bryan, G. L. \& Norman, M. L. 2002, Science, 259, 93

Abramovici, A. et al. 1992, Science, 256, 325

Akerlof, C. et al. 1999, Nature, 398, 400

Aloy, M. A., Janka, J.-T. \& Müller, E. 2005, A\&A, 436, 273

Amati, L. 2006, MNRAS, 372, 233

Amati, L. et al. 2006, A\&A, submitted (astro-ph/0607148)

Amati L., Frontera F., Tavani M. et al., 2002, A\&A, 390, 81

Antonelli, L. A. et al. GCN Circ. 3924

Atkins, R. et al. 2000, ApJ, 533, L119

Atkins, R. et al. 2003, ApJ, 583, 824

Bahcall, J. N. \& Mészáros, P. 2000, PRL, 85, 1362

Bahcall, J. N. \& Waxman, E. 2001, PRD, 64, 023002

Band, D. et al., 1993, ApJ, 413, 281

Band, D. \& Preece, R. D. 2005, ApJ, 627, 319 
Barbier, L. et al. 2006, GCN Circ. \#4397

Baring, M. G. 2006, ApJ, 650, 1004

Baring, M. G. \& Harding, A. K. 1997, ApJ, 491, 663

Barraud C., Daigne, F., Mochkovitch, R. \& Atteia, J. L. 2005, A\&A, 440, 809

Barthelmy, S. D. et al. 2005a, Spa. Sci. Rev. 120, 143

Barthelmy, S. D. et al. 2005b, Nature, 438, 994

Barthelmy, S. D. et al. 2005c, ApJ, 635, L133

Barthelmy, S. D. et al. 2006, GCN Circ \#4401

Barwick, S. W. et al. 2006, PRL, 96, 171101

Belczynski, K., Kalogera, V. \& Bulik, T. 2002, ApJ, 572, 407

Belczynski, K. et al. 2006, ApJ, 648, 1110

Beloborodov, A. M. 2000, ApJ, 539, L25

Beloborodov, A. M. 2003, ApJ, 585, L19

Beloborodov, A. M. 2005, ApJ, 618, L13

Bennett, C. L. et al. 2003, ApJS, 148, 1

Benno, W. et al. 2003, SPIE, 4856, 238

Berger, E. et al. 2003a, Nature, 426, 154

Berger, E., Kulkarni, S. R. \& Frail, D. 2003b, ApJ, 590, 379

Berger, E. et al. 2005a, Nature, 438, 988

Berger, E. et al. 2005b, ApJ, 634, 501

Berger, E. et al. 2005c, ApJ, 642, 979

Berger, E. et al. 2006a, ApJ, submitted (astro-ph/0608498)

Berger, E. et al. 2006b, ApJ, submitted (astro-ph/0611128)

Bhattacharjee, P. \& Gupta, N. 2003, APh, 20, 169

Blake, C. H. et al. 2005, Nature, 435, 181

Blandford, R. D. \& Mckee, C. F. 1976, Phys. of Fluids, 19, 1130

Bloom, J. S. et al. 1999a, Nature, 401, 453

Bloom, J. S., Sigurdsson, S. \& Pols, O. R. 1999b, MNRAS, 305, 763

Bloom, J. S. et al. 2002, ApJ, 572, L45

Bloom, J. S., Frail, D. \& Kulkarni, S. R. 2003, ApJ, 594, 674

Bloom, J. S. et al. 2006a, ApJ, 638, 354

Bloom, J. S. et al. 2006b, ApJ, in press (astro-ph/0607223)

Blustin, A. J. et al. 2006, ApJ, 637, 901

Boër, M. et al. 2006, ApJ, 638, L71

Böttcher, M. \& Dermer, C. D. 1998, ApJ, 499, L131

Bromm, V., Coppi, P. S. \& Larson, R. B. 1999, ApJ, 527, L5

Bromm, V. \& Loeb, A. 2002, ApJ, 575, 111

Bromm, V. \& Loeb, A. 2006, ApJ, 642, 382

Burrows, D. N. 2006, II Nuovo Cimento, submitted

Burrows, D. N. et al. 2005a, Spa. Sci. Rev. 120, 165

Burrows, D. N. et al. 2005b, Sci., 309, 1833

Burrows, D. N. et al. 2006, ApJ, 653, 468

Burrows, D. N. et al. 2007, Phil. Trans. A. in press (astro-ph/0701046)

Butler, N. R. \& Kocevski, D. 2007, ApJ, submitted (astro-ph/0612564)

Campana, S. et al. 2005, ApJ, 625, L23 
Campana, S. et al. 2006a, Nature, 442, 1008

Campana, S. et al. 2006b, A\&A, 454, 113

Campana, S. et al. 2007, ApJ, 654, L17

Casanova, S., Dingus, B. L. \& Zhang, B. 2007, ApJ, in press (astro-ph/0606706)

Cenko, S. B. et al. GCN Circ. \#5155

Chen, H.-W., Prochaska, J. X., Bloom, J. S. \& Thompson, I. B. 2005, ApJ, 634, L25

Chen, S. L., Li, A. \& Wei, D. M. 2006, ApJ, 647, L13

Cheng, K. S., Huang, Y. F. \& Lu, T. 2001, MNRAS, 325, 599

Cheng, K. S. \& Lu, T. 2001, ChJAA, 1, 1

Cheng, L. X. \& Cheng, K. S. 1996, ApJ, 459, L79

Chevalier, R. A. \& Li, Z.-Y. 1999, ApJ, 520, L29

Chevalier, R. A. \& Li, Z.-Y. 2000, ApJ, 536, 195

Chincarini, G. et al. 2005, preprint, (astro-ph/0506453)

Chincarini, G. et al. 2007, in preparation

Ciardi, B. \& Loeb, A. 2000, ApJ, 540, 687

Cobb, B. E. et al. 2006a, ApJ, 645, L113

Cobb, B. E. et al. 2006b, ApJ, 651, L85

Coburn, W. \& Boggs, S. E. 2003, Nature, 423, 415

Colgate, S. A. 1974, ApJ, 187, 333

Connaughton, V. 2002, ApJ, 567, 1028

Costa, E. et al. 1997, Nature, 387, 783

Covino, S. et al. 2006, II Nuovo Cimento, in press (astro-ph/0612643)

Cui, X.-H., Liang, E.-W. \& Lu, R.-J. 2005, ChJAA, 5, 151

Cusumano, G. et al. 2006a, Nature, 440, 164

Cusumano, G. et al. 2006b, ApJ, 639, 316

Cusumano, G. et al. 2006c, A\&A, in press (astro-ph/0610570)

Dado, S., Dar, A. \& De Rujula, A. 2006, ApJ, 646, L21

Dai, X. et al. 2007, ApJ, in press (astro-ph/0609269)

Dai, X. \& Zhang, B. 2005, ApJ, 621, 875

Dai, Z. G. 2004, ApJ, 606, 1000

Dai, Z. G. \& Cheng, K. S. 2001, ApJ, 558, L109

Dai, Z. G. \& Gou, L. J. 2001, ApJ, 552, 72

Dai, Z. G., Liang, E. W. \& Xu, D. 2004, ApJ, 612, L101

Dai, Z. G. \& Lu, T. 1998a, PRL, 81, 4301

Dai, Z. G. \& Lu, T. 1998b, A\&A, 333, L87

Dai, Z. G. \& Lu, T. 1998c, MNRAS, 298, 87

Dai, Z. G. \& Lu, T. 1999, ApJ, 519, L155

Dai, Z. G. \& Lu, T. 2001, ApJ, 551, 249

Dai, Z. G. \& Lu, T. 2002a, ApJ, 580, 1013

Dai, Z. G. \& Lu, T. 2002b, ApJ, 565, L87

Dai, Z. G.. Wang, X. Y., Wu, X. F. \& Zhang, B. 2006a, Science, 311, 1127

Dai, Z. G. \& Wu, X. F. 2003, ApJ, 591, L21

Dai, Z. G., Wu, X. F., Wang, X. Y., Huang, Y. F. \& Zhang, B. 2005, ApJ, 629, L81

Dai, Z. G., Zhang, B. \& Liang, E. W. 2006b, preprint, (astro-ph/0604510)

Daigne, F. \& Mochkovitch, R. 1998, MNRAS, 296, 275 
D'Alessio, V., Piro, L. \& Rossi, E. M. 2006, A\&A, 460, 653

Della Valle, M. et al. 2003, A\&A, 406, L33

Della Valle, M. et al. 2006a, ApJ, 642, L103

Della Valle, M. et al. 2006b, Nature, 444, 1050

De Pasquale, M. et al. 2006a, MNRAS, 365, 1031

De Pasquale, M. et al. 2006b, MNRAS, submitted

Derishev, E. V., Kocharovsky, V. V. \& Kocharovsky, Vl. V. 1999, ApJ, 521, 640

Dermer, C. D. 2002, ApJ, 574, 65

Dermer, C. D. 2004, ApJ, 614, 284

Dermer, C. D. 2006, in Gamma-ray bursts in the Swift era, AIP Conf. Proc. V836, 97

Dermer, C. D. \& Atoyan, A. 2006, ApJ, 643, L13

Dermer, C. D., Chiang, J. \& Böttcher, M. 1999, ApJ, 513, 656

Dermer, C. D., Chiang, J. \& Mitman, K. E. 2000, ApJ, 537, 785

De Ugarte Postigo, A. et al. 2006, ApJ, 648, L83

Dingus, B. L. et al. AIP Conf. Proc. 727, 131

Donaghy, T. Q. et al. 2006, ApJ, submitted (astro-ph/0605570)

Donaghy, T. Q., Lamb, D. Q. \& Graziani, C. 2004, AIP Conf. Proc. 727, 47

Dong, Y.-M. \& Qin, Y.-P. 2005, MNRAS, 358, 1267

Drenkhahn, G. \& Spruit, H. C. 2002, A\&A, 391, 1141

Dyks, J., Zhang, B. \& Fan, Y. Z. 2005, preprint, astro-ph/0511699

Eichler, D. \& Granot, 2006, ApJ, 641, L5

Eichler, D. \& Levinson, A. 2004, ApJ, 614, L13

Eichler, D. \& Levinson, A. 2006, ApJ, 649, L5

Eichler, D., Livio, M., Piran, T. \& Schramm, D. N. 1989, Nature, 340, 126

Faber, J. A., Baumgarte, T. W., Shapiro, S. L. \& Taniguchi, K. 2006, ApJ, 641, L93

Falcone, A. D. et al. 2006, ApJ, 641, 1010

Fan, Y.-Z., Dai, Z.-G., Huang, Y.-F. \& Lu, T. 2002, ChJAA, 2, 449

Fan, Y. Z. \& Piran, T. 2006a, MNRAS, 369, 197

Fan, Y. Z. \& Piran, T. 2006b, MNRAS, 370, L24

Fan, Y. Z., Piran, T., Xu, D. 2006, JCAP, 9, 13

Fan, Y. Z. \& Wei, D. M. 2004, ApJ, 615, L69

Fan, Y. Z. \& Wei, D. M. 2005, MNRAS, 364, L42

Fan, Y. Z., Wei, D. M. \& Wang, C. F. 2004a, A\&A, 424, 477

Fan, Y. Z., Wei, D. M. \& Zhang, B. 2004b, MNRAS, 354, 1031

Fan, Y. Z. \& Xu, D. 2006, MNRAS, 372, L19

Fan, Y. Z., Zhang, B., Kobayashi, S. \& Mészáros, P. 2005a, ApJ, 628, 867

Fan, Y. Z., Zhang, B. \& Wei, D. M. 2005b, ApJ, 628, 298

Fan, Y. Z., Zhang, B. \& Wei, D. M. 2005c, ApJ, 628, L25

Fan, Y. Z., Zhang, B. \& Wei, D. M. 2005e, ApJ, 629, 334

Fan, Y. Z., Zhang, B. \& Proga, D. 2005d, ApJ, 635, L129

Fenimore, E. E., Madras, C. D. \& Nayakshin, S. 1996, ApJ, 473, 998

Fenimore, E. E. \& Ramirez-Ruiz, E. 2000, preprint, (astro-ph/0004176)

Ferrero, P. et al. 2006, AJ, submitted (astro-ph/0610255)

Filippenko, A. V. 1997, ARA\&A, 35, 309

Finn, L. S., Mohanty, S. D. \& Romano, J. D. 1999, PRD, 60, 121101 
Firmani, C., Avila-Reese, V., Ghisellini, G. \& Tutukov, A. V. 2004, ApJ, 611, 1033

Firmani, C., Avila-Reese, V., Ghisellini, G. \& Ghirlanda, G. 2006a, MNRAS, 370, 185

Firmani, C., Avila-Reese, V., Ghisellini, G. \& Ghirlanda, G. 2006b, MNRAS, 372, L28

Firmani, C., Ghisellini, G., Ghirlanda, G. \& Avila-Reese, V. 2005, MNRAS, 360, L1

Fishman, G. J. \& Meegan, C. A. 1995, ARA\&A, 33, 415

Fox, D. W. et al. 2003a, Nature, 422, 284

Fox, D. W. et al. 2003b, ApJ, 596, L5

Fox, D. B. et al. 2005, Nature, 437, 845

Fox, D. B. \& Mészáros, P. 2006, New J. Phys. 8, 199

Fragile, P. C., Mathews, G. J., Poirier, J. \& Totani, T. 2004, APh, 20, 591

Frail, D. A. et al. 1997, Nature, 389, 261

Frail, D. A. et al. 2001, ApJ, 562, L55

Frail, D. A. et al. 2003, AJ, 125, 2299

Frail, D. A. et al. 2006, ApJ, 646, L99

Freedman, D. L. \& Waxman, E. 2001, ApJ, 547, 922

Friedman, A. S. \& Bloom, J. S. 2005, ApJ, 627, 1

Fruchter, A. et al. 2006, Nature, 441, 436

Fryer, C., Holz, D. \& Hughes, S. 2002, ApJ, 565, 430

Fryer, C. L., Woosley, S. E. \& Hartmann, D. H. 1999, ApJ, 526, 152

Fujimoto, M.-K. et al. 2005, ARAOJ, 7, 37

Fynbo, J. P. U. et al. 2004, ApJ, 609, 962

Fynbo, J. P. U. et al. 2006a, Nature, 444, 1047

Fynbo, J. P. U. et al. 2006b, GCN Circ. \#5651

Galama, T. J. et al. 1998, Nature, 395, 670

Gal-Yam, A. et al. 2006, Nature, 444, 1053

Gao, W. H. \& Fan, Y. Z. 2006, ChJAA, 6, 513

Gehrels, N. et al. 2004, ApJ, 611, 1005

Gehrels, N. et al. 2005, Nature, 437, 851

Gehrels, N. et al. 2006, Nature, 444, 1044

Gehrels, N. \& Michelson, P. 1999, APh, 11, 277

Gendre, B. \& Boer, M. 2005, A\&A, 430, 465

Gendre, B., Galli, A. \& Piro, L. 2006a, A\&A, submitted (astro-ph/0612178)

Gendre, B. et al. 2006b, A\&A, in press (astro-ph/0603431)

Ghirlanda, G., Ghisellini, G. \& Celotti, A. 2004a, A\&A, 422, L55

Ghirlanda, G., Ghisellini, G. \& Lazzati, D. 2004b, ApJ, 616, 331

Ghirlanda, G., Ghisellini, G., Lazzati, D. \& Firmani, C. 2004c, ApJ, 613, L13

Ghirlanda, G., et al. 2006, A\&A, 452, 839

Ghisellini, G., Celotti, A. \& Lazzati, D. 2000, MNRAS, 313, L1

Ghisellini, G. et al. 2006, MNRAS, 372, 1699

Ghisellini, G., Ghirlanda, G. \& Tavecchio, F. 2007, MNRAS, in press (astro-ph/0608555)

Giannios, D. 2006, A\&A, 455, L5

Goad, M. R. et al. 2006, A\&A, 449, 89

Godet, O. et al. 2006, A\&A, 452, 819

Gonzalez, M. M., Dingus, B. L., Kaneko, Y., Preece, R. D., Dermer, C. D., Briggs, M. S. 2003, Nature, 424, 749 
Gonzalez-Garcia, M. C. \& Halzen, F. 2006, preprint, hep-ph/0611359

Goodman, J. 1986, ApJ, 308, L47

Gou, L. J., Fox, D. B. \& Mészáros, P. 2007, ApJ, submitted (astro-ph/0612256)

Gou, L. J., Mészáros, P., Abel, T. \& Zhang, B. 2004, ApJ, 604, 508

Granot, J. \& Guetta, D. 2003, ApJ, 598, L11

Granot, J., Königl, A. \& Piran, T. 2006, MNRAS, 370, 1946

Granot, J. \& Kumar, P. 2003, ApJ, 591, 1075

Granot, J. \& Kumar, P. 2006, MNRAS, 366, L13

Granot, J., Nakar, E. \& Piran, T. 2003, Nature, 426, 138

Granot, J., Panaitescu, A., Kumar, P. \& Woosley, S. E. 2002, ApJ, 570, L61

Greiner, J. 2006, personal webpage http://www.mpe.mpg.de/ jcg/grbgen.html

Grindlay, J., Portegies, Z. \& McMillan, S. 2006, Nature Phys. 2, 116

Grupe, D. et al. 2006a, ApJ, 653, 462

Grupe, D. et al. 2006b, ApJ, submitted (astro-ph/0611240)

Guetta, D., Perna, R., Stella, L. \& Vietri, M. 2004, ApJ, 615, L73

Guetta, D. \& Piran, T. 2006, A\&A, 453, 823

Guetta, D., Piran, T. \& Waxman, E. 2005, ApJ, 619, 412

Guidorzi, C. et al. 2005, MNRAS, 363, 315

Guidorzi, C. et al. 2006, MNRAS, 371, 843

Gupta, N. \& Zhang, B. 2007a, AstroParticle Phys., in press (astro-ph/0606744)

Gupta, N. \& Zhang, B. 2007b, in preparation

Haislip, J. B. et al. 2006, Nature, 440, 181

Hakkila, J. \& Giblin, T. W. 2006, ApJ, 646, 1086

Harrison, F. A. et al. 1999, ApJ, 523, L121

Hededal, C. B. \& Nishikawa, K.-I. 2005, ApJ, 623, L89

Heise, J., in'tZand, J., Kippen, R. M., Woods, P. M. 2001, in Gamma-Ray Bursts in the Afterglow Era:

Prof. Int. Workshop held in Rome, 17-20 Oct. 2000 (eds. E. Costa, F. Frontera \& J. Hjorth), 16

Heyl, J. S. \& Perna, R. 2003, ApJ, 586, L13

Hill, J. E. et al. 2006, ApJ, 639, 303

Hill, G. C. 2006, to appear J. of Phys. Conf. Ser. (astro-ph/0611773)

Hinton, J. A. 2004, New Astron. Rev. 48, 331

Hjorth, J. et al. 2003, Nature, 423, 847

Hjorth, J. et al. 2005, Nature, 437, 859

Holland, S. T. et al. 2003, AJ, 125, 2291

Horan, D. et al. AIP Conf Proc. 745, 591

Horváth, I. 1998, ApJ, 508, 757

Horváth, I. et al. 2006, A\&A, 447, 23

Huang, K. Y. et al. 2007, ApJ, 654, L25

Huang, Y. F. \& Cheng, K. S. 2003, MNRAS, 341, 263

Huang, Y. F., Cheng, K. S. \& Gao, T. T. 2006, ApJ, 637, 873

Huang, Y. F., Dai, Z. G. \& Lu, T. 1998, A\&A, 336, L69

Huang, Y. F., Dai, Z. G. \& Lu, T. 1999, MNRAS, 309, 513

Huang, Y. F., Dai, Z. G. \& Lu, T. 2002, MNRAS, 332, 735

Huang, Y. F., Gou, L. J., Dai, Z. G. \& Lu, T. 2000, ApJ, 543, 90

Huang, Y. F., Lu, Y., Wong, A. Y. L. \& Cheng, K. S. 2007, ChJAA, in press 
Huang, Y. F., Wu, X. F., Dai, Z. G., Ma, H. T. \& Lu, T. 2004, ApJ, 605, 300

Hurkett, C. P. et al. 2006, MNRAS, 368, 1101

Hurley, K. et al. 1994, Nature, 372, 652

Hurley, K. et al. 2005, Nature, 434, 1098

in't Zand, J. J. M. et al. 2001, ApJ, 559, 710

Ioka, K., Kobayashi, S. \& Mészáros, P. 2004, ApJ, 613, L17

Ioka, K., Kobayashi, S. \& Zhang, B. 2005, ApJ, 631, 429

Ioka, K., Mészáros, P. 2005, ApJ, 619, 684

Ioka, K. \& Nakamura, T. 2001, ApJ, 554, L163

Ioka, K., Toma, K., Yamazaki, R. \& Nakamura, T. 2006, A\&A, 458, 7

Jakobsson, P. et al. 2004, ApJ, 617, L21

Jakobsson, P. et al. 2006a, A\&A, 447, 897

Jakobsson, P. et al. 2006b, preprint, (astro-ph/0602071)

Jelinek, M. et al. 2006, A\&A, 454, L119

Jin, Z.-P. \& Wei, D.-M. 2004, ChJAA, 4, 473

Jin, Z. P., Yan, T., Fan, Y. Z. \& Wei, D. M. 2006, preprint, (astro-ph/0610010)

Kalemci, E., Boggs, S. E., Kouveliotou, C., Finger, M. \& Baring, M. G. 2007, ApJ, in press (astro$\mathrm{ph} / 0610771)$

Kann, D. A., Zeh, A. \& Klose, S. 2006, ApJ, 641, 993

Kato, T. 2005, Phys. of Plasmas, 12, 080705

Katz, U. F. 2006, Nucl. Instrum. Meth, A567, 457

Kawai, N. et al. 2006, Nature, 440, 184

King, A. et al. 2005, ApJ, 630, L113

King, A., Olsson, E. \& Davies, M. B. 2007, MNRAS, 374, L34

Kippen, R. M. et al. 2002, preprint, astro-ph/0203114

Klotz, A. et al. 2006, A\&A, 451, L39

Kluzniak, W. \& Ruderman, M. 1998, ApJ, 505, L113

Kobayashi, S. 2000, ApJ, 545, 807

Kobayashi, S., Ryde, F. \& MacFadyen, A. 2002, ApJ, 577, 302

Kobayashi, S. \& Mészáros, P. 2003, ApJ, 589, 861

Kobayashi, S., Mészáros, P. \& Zhang, B. 2004, ApJ, 601, L13

Kobayashi, S., Piran, T. \& Sari, R. 1997, ApJ, 490, 92

Kobayashi, S., Piran, T. \& Sari, R. 1999, ApJ, 513, 699

Kobayashi, S. \& Sari, R. 2000, ApJ, 542, 819

Kobayashi, S. \& Sari, R. 2001, ApJ, 551, 934

Kobayashi, S. \& Zhang, B. 2003a, ApJ, 582, L75

Kobayashi, S. \& Zhang, B. 2003b, ApJ, 597, 455

Kobayashi, S. \& Zhang, B. 2007, ApJ, in press (astro-ph/0608132)

Kobayashi, S., Zhang, B., Mészáros, P. \& Burrows, D. 2007, ApJ, in press (astro-ph/0506157)

Kocevski, D., Ryde, F. \& Liang, E. 2003, ApJ, 596, 389

Kochanek, C. S. \& Piran, T. 1993, ApJ, 417, L17

Kouveliotou, C. et al. 1993, ApJ, 413, L101

Krimm, H. et al. 2006a, GCN Circ \#5704

Krimm, H. et al. 2006b, ApJ, 648, 1117

Kubo, H. et al. 2004, New Astron. Rev. 48, 323 
Kulkarni, S. R. 2005, preprint (astro-ph/0510256)

Kulkarni, S. R. et al. 1998, Nature, 395, 663

Kulkarni, S. R. et al. 1999, Nature, 398, 389

Kumar, P. 1999, ApJ, 523, L113

Kumar, P. \& Granot, J. 2003, ApJ, 591, 1086

Kumar, P. \& Panaitescu, A. 2000, ApJ, 541, L51

Kumar, P. \& Panaitescu, A. 2003, MNRAS, 346, 905

Kumar, P. et al. 2006, MNRAS, 367, L52

Kumar, P. et al. 2007, MNRAS, in press

Lamb, D. Q., Donaghy, T. Q. \& Graziani, C. 2005a, ApJ, 620, 355

Lamb, D. Q. et al. preprint, (astro-ph/0507362)

Lamb, D. Q. \& Reichart, D. E. 2000, ApJ, 536, 1

La Parola, V. et al. 2006, A\&A, 454, 753

Lazzati, D. et al. 2002, A\&A, 396, L5

Lazzati, D. \& Begelman, M. C. 2006, ApJ, 641, 972

Lazzati, D. \& Perna, R. 2007, MNRAS, in press (astro-ph/0610730)

Lazzati, D., Ramirez-Ruiz, E. \& Ghisellini, G. 2001, A\&A, 379, L39

Lee, W. H., Ramirez-Ruiz, E. \& Granot, J. 2005, ApJ, 630, L165

Levan, A. J. et al. 2006a, ApJ, 648, L9

Levan, A. J. et al. 2006b, ApJ, 648, 1132

Levan, A. J. et al. 2006c, MNRAS, 368, L1

Levinson, A. \& Eichler, D. 2005, ApJ, 629, L13

Li, H., Dai, Z. G. \& Zhang, X. 2005, PRD, 71, 113003

Li, H., Su, M., Fan, Z., Dai, Z. \& Zhang, X. 2006, preprint, (astro-ph/0612060)

Li, L.-X. 2007, MNRAS, in press (astro-ph/0605387)

Li, L.-X. \& Paczyński, B. 1998, ApJ, 507, L59

Li, L.-X. \& Paczyński, B. 2006, MNRAS, 366, 219

Li, W. et al. 2003a, ApJ, 596, L9

Li, Z., Dai, Z. G. \& Lu, T. 2002, A\&A, 396, 303

Li, Z., Dai, Z. G., Lu, T. \& Song, L. M. 2003b, ApJ, 599, 380

Li, Z. \& Song, L. M. 2004, ApJ, 614, L17

Li, Z. \& Waxman, E. 2006, ApJ, 651, 328

Li, Z.-Y. \& Chevalier, R. A. 2001, ApJ, 551, 940

Liang, E. \& Nishimura, K. 2004, PRL, 92, 175005

Liang, E. W. \& Dai, Z. G. 2004, ApJ, 608, L67

Liang, E. W., Dai, Z. G. \& Wu, X. F. 2004, ApJ, 606, L29

Liang, E. W., Xie, G. Z. \& Su, C. Y. 2002, PASJ, 54, 1

Liang, E. W. \& Zhang, B. 2005, ApJ, 633, 611

Liang, E. W. \& Zhang, B. 2006a, ApJ, 638, L67

Liang, E. W. \& Zhang, B. 2006b, MNRAS, 369, L37

Liang, E. W. et al. 2006a, ApJ, 646, 351

Liang, E. W. et al. 2006b, ApJ, 653, L81

Liang, E. W., Zhang, B., Virgili, F. \& Dai, Z. G. 2006c, ApJ, submitted (astro-ph/0605200)

Lin, J. R., Zhang, S. N. \& Li, T. P. 2004, ApJ, 605, 819

Lipkin, Y. M. et al. 2004, ApJ, 606, 381 
Lipunov, V. M., Postnov, K. A. \& Prokhorov, M. E. 2001, Astron. Rep. 45, 236

Lithwick, Y. \& Sari, R. 2001, ApJ, 555, 540

Livio, M. \& Waxman, E. 2000, ApJ, 538, 187

Lloyd, N. M. \& Petrosian, V. 2000, ApJ, 543, 722

Lloyd, N. M., Petrosian, V., Mallozzi R.S. 2000, ApJ, 534, 227

Lloyd-Ronning, N. M., Dai, X. \& Zhang, B. 2004, ApJ, 601, 371

Lloyd-Ronning, N. M., Fryer, C. L. \& Ramirez-Ruiz, E. 2002, ApJ, 574, 554

Lloyd-Ronning, N. M. \& Zhang, B. 2004, ApJ, 613, 477

Loeb, A. \& Barkana, R. 2001, ARA\&A, 39, 19

Lorenz, E. 2004, New Astron. Rev. 48, 339

Lu, T., Huang, Y. F., Dai, Z. G. \& Wei, D. M. 2004, in Cosmic Gamma-Ray Sources (eds. K. S. Cheng \& G. E. Romero), Kluwer Academic Publishers, p225

Lyutikov, M. 2006a, MNRAS, 369, L5

Lyutikov, M. 2006b, New J. Phys., 8, 119

MacFadyen, A. I., Ramirez-Ruiz, E. \& Zhang, W. 2005, preprint, (astro-ph/0510192)

MacFadyen, A. I. \& Woosley, S. E. 1999, ApJ, 524, 262

MacFadyen, A. I., Woosley, S. E. \& Heger, A. 2001, ApJ, 550, 410

Malesani, D. et al. 2004, ApJ, 609, L5

Mangano, V. et al. 2007a, ApJ, 654, 403

Mangano, V. et al. 2007b, A\&A, submitted

Mason, K. O. et al. 2006, ApJ, 639, 311

Mazzali, P. A. et al. 2006, Nature, 442, 1018

McMahon, E., Kumar, P. \& Piran, T. 2006, MNRAS, 366, 575

Medvedev, M. V. 2000, ApJ, 540, 704

Medvedev, M. V. 2006, ApJ, 651, L9

Medvedev, M. V. \& Loeb, A. 1999, ApJ, 526, 697

Mészáros, P. 2002, ARA\&A, 40, 137

Mészáros, P. 2006, Rev. Prog. Phys. 2006, 69, 2259

Mészáros, P., Laguna, P. \& Rees, M. J. 1993, ApJ, 415, 181

Mészáros, P., Ramirez-Ruiz, E., Rees, M. J. \& Zhang, B. 2002, ApJ, 578, 812

Mészáros, P. \& Rees, M. J. 1992, MNRAS, 257, 29P

Mészáros, P. \& Rees, M. J. 1993, ApJ, 405, 278

Mészáros, P. \& Rees, M. J. 1994, MNRAS, 269, L41

Mészáros, P. \& Rees, M. J. 1997a, ApJ, 476, 232

Mészáros, P. \& Rees, M. J. 1997b, ApJ, 482, L29

Mészáros, P. \& Rees, M. J. 1999, MNRAS, 306, L39

Mészáros, P. \& Rees, M. J. 2000, ApJ, 530, 292

Mészáros, P. \& Rees, M. J. 2001, ApJ, 556, L37

Mészáros, P., Rees, M. J. \& Papathanassiou, H. 1994, ApJ, 432, 181

Mészáros, P., Rees, M. J. \& Wijers, R. A. M. J. 1998, ApJ, 499, 301

Mészáros, P. \& Waxman, E. 2001, PRL, 87, 171102

Milgrom, M. \& Usov, V. V. 1995, ApJ, 449, L37

Milosavljevic, M. \& Nakar, E. 2006a, ApJ, 641, 978

Milosavljevic, M. \& Nakar, E. 2006b, ApJ, 651, 979

Mirabal, N., Halpern, J. P., An, D. et al. 2006, ApJ, 643, L21 
Mizuno, Y., Yamada, S., Koide, S. \& Shibata, K. 2004a, ApJ, 606, 395

Mizuno, Y., Yamada, S., Koide, S. \& Shibata, K. 2004b, ApJ, 615, 389

Mizuta, A., Yamasaki, T., Nagataki, S. \& Mineshige, S. 2006, ApJ, 651, 960

Modjaz, M. et al. 2006, ApJ, 645, L21

Modjaz, M. et al. 2007, AJ, submitted (astro-ph/0701246)

Molinari, E. et al. 2006, preprint (astro-ph/0612607)

Monfardini, A. et al. 2006, ApJ, 648, 1125

Morsony, B. J., Lazzati, D. \& Begelman, M. C. 2006, ApJ, submitted (astro-ph/0609254)

Mosquera Cuesta, H. J. et al. preprint, (astro-ph/0609262)

Mukherjee, S. et al. 1998, ApJ, 508, 314

Mundell et al. 2006, ApJ, submitted (astro-ph/0610660)

Murase, K., Ioka, K., Nagataki, S. \& Nakamura, T. 2006, ApJ, 651, L5

Murase, K. \& Nagataki, S. 2006, PRL, 97, 051101

Nakar, E., Gal-Yam, A. \& Fox, D. B. 2006a, ApJ, 650, 281

Nakar, E., Gal-Yam, A., Piran, T. \& Fox, D. B. 2006b, ApJ, 640, 849

Nakar, E. \& Granot, J. 2006, preprint (astro-ph/0606011)

Nakar, E. \& Piran, T. 2002, MNRAS, 330, 920

Nakar, E. \& Piran, T. 2004, MNRAS, 353, 647

Nakar, E. \& Piran, T. 2005, MNRAS, 360, L73

Nakar, E., Piran, T. \& Granot, J. 2003, New Astron., 8, 495

Narayan, R., Paczyński, B. \& Piran, T. 1992, ApJ, 395, L8

Nardini, M. et al. 2006, A\&A, 451, 821

Nava, L. et al. 2006, A\&A, 450, 471

Nishikawa, K.-I. et al. 2003, ApJ, 595, 555

Nishikawa, K.-I. et al. 2005, ApJ, 622, 927

Norris, J. 2002, ApJ, 579, 386

Norris, J. P. \& Bonnell, J. T. 2006, ApJ, 643, 266

Norris, J. P. \& Gehrels, N. 2007, in preparation

Norris, J., Marani, G. F. \& Bonnell, J. T. 2000, ApJ, 534, 248

Nousek, J. A. et al. 2006, ApJ, 642, 389

Oates, S. R. et al. 2006, MNRAS, 372, 327

O'Brien, P. T., Willingale, R., Osborne, J. P. \& Goad, M. R. 2006a, New J. Phys. 8, 121

O'Brien, P. T. et al. 2006b, ApJ, 647, 1213

Ofek, E. O. et al. 2006, GCN Circ. \#5123

Paczyński, B. 1986, ApJ, 308, L43

Paczyński, B. 1998, ApJ, 494, L45

Paczyński, B. 1991, AcA, 41, 257

Paczyński, B. \& Xu, G. 1994, ApJ, 427, 708

Page, K. L. et al. 2006a, ApJ, 637, L13

Page, K. L. et al. 2006b, ApJ, submitted

Palmer, D. et al. 2005, Nature, 434, 1107

Palmer, D. et al. 2006, GCN Circ. \#5076

Panaitescu, A. 2005a, MNRAS, 363, 1409

Panaitescu, A. 2005b, MNRAS, 362, 921

Panaitescu, A. 2006a, MNRAS, 367, L42 
Panaitescu, A. 2006b, MNRAS, submitted (astro-ph/0612170)

Panaitescu, A. \& Kumar, P. 2000, ApJ, 543, 66

Panaitescu, A. \& Kumar, P. 2001, ApJ, 560, L49

Panaitescu, A. \& Kumar, P. 2002, ApJ, 571, 779

Panaitescu, A. \& Kumar, P. 2003, ApJ, 592, 390

Panaitescu, A. \& Kumar, P. 2004, MNRAS, 353, 511

Panaitescu, A., Kumar, P. \& Narayan, R. 2001, ApJ, 561, L171

Panaitescu, A. \& Mészáros, P. 2000, ApJ, 544, L17

Panaitescu, A., Mészáros, P., Gehrels, N., Burrows, D. \& Nousek, J. 2006a, MNRAS, 366, 1357

Panaitescu, A. et al. 2006b, MNRAS, 369, 2059

Panaitescu, A., Mészáros, P. \& Rees, M. J. 1998, ApJ, 503, 314

Panaitescu, A., Spada, M. \& Mészáros, P. 1999, ApJ, 522, L105

Pe'er, A., Mészáros, P. \& Rees, M. J. 2005, ApJ, 635, 476

Pe'er, A., Mészáros, P. \& Rees, M. J. 2006a, ApJ, 642, 995

Pe'er, A., Mészáros, P. \& Rees, M. J. 2006b, ApJ, 652, 482

Pe'er, A. \& Waxman, E. 2004a, ApJ, 613, 448

Pe'er, A. \& Waxman, E. 2004b, ApJ, 603, L1

Pe'er, A. \& Waxman, E. 2005, ApJ, 628, 857

Pe'er. A. \& Wijers, R. A. M. J. 2006, ApJ, 643, 1036

Pe'er, A. \& Zhang, B. 2006, ApJ, 653, 454

Peng, F., Königl, A. \& Granot, J. 2005, ApJ, 626, 966

Perna, R., Armitage, P. J. \& Zhang, B. 2006, ApJ, 636, L29

Perna, R. \& Belczynski, K. 2002, ApJ, 570, 252

Perna, R., Sari, R. \& Frail, D. 2003, ApJ, 594, 379

Phinney, E. S. 1991, ApJ, 380, L17

Pian, E. et al. 2001, A\&A, 372, 456

Pian, E. et al. 2006, Nature, 442, 1011

Pilla, R. P. \& Loeb, A. 1998, ApJ, 494, L167

Piran, T. 1999, Phys. Rep. 314, 575

Piran, T. 2005, Rev. Mod. Phys. 76, 1143

Piro, A. L. \& Pfahl, E. 2007, ApJ, in press (astro-ph/0610696)

Piro, L. et al. 2005, ApJ, 623, 314

Pittori, C., Tavani, M. 2004, AAS HEAD Meeting \#8, \#16.05

Plaga, R. 1995, Nature, 374, 430

Preece R. D. et al., 2000, ApJS, 126, 19

Price, D. J. \& Rosswog, S. 2006, Sience, 312, 5774

Prochaska, J. X. et al. 2004, ApJ, 611, 200

Proga, D. \& Begelman, M. 2003, ApJ, 592, 767

Proga, D., MacFadyen, A. I., Armitage, P. J. \& Begelman, M. C. 2003, ApJ, 599, L5

Proga, D. \& Zhang, B. 2006, MNRAS, 370, L61

Qin, Y.-P. \& Dong, Y.-M. 2005, MNRAS, 358, 1320

Qin, Y.-P. \& Lu, R.-J. 2005, MNRAS, 362, 1085

Qin, Y.-P., Su, C.-Y., Fan, J. H. \& Gupta, A. C. 2006a, PRD, 74, 063005

Qin, Y.-P. et al. 2000, PASJ, 52, 759

Qin, Y.-P. et al. 2006b, Chi. Phys. 15, 1645 
Rachen, J. P. \& Mészáros, P. 1998, PRD, 58, 123005

Racusin, J. L. et al. 2006, GCN Circ. \#5378

Ramirez-Ruiz, E. 2005, MNRAS, 363, L61

Ramirez-Ruiz, E., Merloni, A. \& Rees, M. J. 2001, MNRAS, 331, 197

Ramirez-Ruiz, E., Celotti, A. \& Rees, M. J. 2002a, MNRAS, 337, 1349

Ramirez-Ruiz, E., Lazzati, D. \& Blain, A. W. 2002b, ApJ, 565, L9

Ramirez-Ruiz, E., Garcia-Segura, G., Salmonson, J. D. \& Perez-Rendon, B. 2005, ApJ, 631, 435

Rampp, M., Müller, E. \& Ruffert, M. 1998, A\&A, 332, 969

Razzaque, S. \& Mészáros, P. 2006a, ApJ, 650, 998

Razzaque, S. \& Mészáros, P. 2006b, JCAP, 06, 006

Razzaque, S., Mészáros, P. \& Waxman, E. 2003, PRL, 90, 241103

Razzaque, S., Mészáros, P. \& Waxman, E. 2004b, PRD, 69, 023001

Razzaque, S., Mészáros, P. \& Zhang, B. 2004a, ApJ, 613, 1072

Razzaque, S. \& Zhang, B. 2007, in preparation

Rees, M. J. \& Mészáros, P. 1992, MNRAS, 258, 41P

Rees, M. J. \& Mészáros, P. 1994, ApJ, 430, L93

Rees, M. J. \& Mészáros, P. 1998, ApJ, 496, L1

Rees, M. J. \& Mészáros, P. 2005, ApJ, 628, 847

Reichart, D. 1999, ApJ, 521, L111

Reichart, D. 2005, preprint, (astro-ph/0508529)

Reichart, D. et al. 2001, ApJ, 552, 57

Retter, A. et al. 2005, GCN Circ. \#3788

Rhoads, J. E. 1997, ApJ, 487, L1

Rhoads, J. E. 1999, ApJ, 525, 737

Rol, E. et al. 2005, ApJ, 624, 868

Romano, P. et al. 2006a, A\&A, 450, 59

Romano, P. et al. 2006b, A\&A, 456, 917

Roming, P. W. A. et al. 2005, Spa. Sci. Rev. 120, 95

Roming, P. W. A. et al. 2006a, ApJ, 652, 1416

Roming, P. W. A. et al. 2006b, ApJ, 651, 985

Roming, P. W. A. et al. 2006c, Nuovo Cimento, submitted

Rossi, E., Lazzati, D. \& Rees, M. J. 2002, MNRAS, 332, 945

Rossi, E., Lazzati, D., Salmonson, J. D. \& Ghisellini, G. 2004, MNRAS, 354, 86

Rossi, E. \& Rees, M. J. 2003, MNRAS, 339, 881

Rosswog, S. 2005, ApJ, 634, 1202

Rosswog, S. 2006, MNRAS, submitted (astro-ph/0611440)

Rosswog, S., Ramirez-Ruiz, E. \& Davies, M. B. 2003, MNRAS, 334, 481

Ruderman, M. A., Tao, L. \& Kluzniak, W. 2000, ApJ, 542, 243

Ruffert, M. \& Janka, H. Th. 1999, A\&A, 344, 573

Rutledge, R. E. \& Fox, D. B. 2004, MNRAS, 350, 1288

Ryde, F. 2005, ApJ, 625, L95

Ryde, F. et al. 2006, ApJ, 652, 1400

Ryde, F., Pe'er, A. \& Mészáros, P. 2007, in preparation

Rykoff, E. S. et al. 2006, ApJ, 638, L5

Sago, N., Ioka, K., Nakamura, T. \& Yamazaki, R. 2004, PRD, 70, 104012 
Sakamoto, T. et al. 2005, ApJ, 629, 311

Sakamoto, T. et al. 2006a, ApJ, 636, L73

Sakamoto, T. et al. 2006b, ApJ, submitted

Salmonson, J. D. 2000, ApJ, 569, 682

Salmonson, J. D. 2003, ApJ, 592, 1002

Salvaterra, R. \& Chincarini, C. 2006, ApJL, submitted (astro-ph/0612278)

Sari, R. \& Esin, A. A. 2001, ApJ, 548, 787

Sari, R. \& Mészáros, P. 2000, ApJ, 535, L33

Sari, R. \& Piran, T. 1995, ApJ, 455, L143

Sari, R. \& Piran, T. 1999a, ApJ, 517, L109

Sari, R. \& Piran, T. 1999b, ApJ, 520, 641

Sari, R., Piran, T. \& Halpern, J. P. 1999, ApJ, 519, L17

Sari, R., Piran, T. \& Narayan, R. 1998, ApJ, 497, L17

Sato, G. et al. 2007, ApJ, in press (astro-ph/0611148)

Schady, P. et al. 2006a, MNRAS, submitted (astro-ph/0611081)

Schady, P. et al. 2006b, ApJ, 643, 276

Schaefer, B. E. 2003, ApJ, 583, L67

Schaefer, B. E. 2006, ApJ, 642, L25

Schaefer, B. E. 2007, ApJ, in press (astro-ph/0612285)

Schaefer, B. E., Deng, M. \& Band, D. L. 2001, ApJ, 563, L123

Schaefer, B. E. \& Xiao, L. 2006, preprint, (astro-ph/0608441)

Schmidt, M. 2001, ApJ, 552, 36

Shao, L. \& Dai, Z. G. 2005, ApJ, 633, 1027

Shao, L. \& Dai, Z. G. 2006, ApJ, submitted

Shen, R., Kumar, P. \& Robinson, E. L. 2006, MNRAS, 371, 1441

Shen, R.-F., Song, L.-M. \& Li, Z. 2005, MNRAS, 362, 59

Shibata, M., Taniguchi, K. \& Uryu, K. 2005, PRD, 71, 084021

Shibata, M. \& Uryu, K. 2006, PRD, 74, 121503

Soderberg, A. M. \& Ramirez-Ruiz, E. 2003, MNRAS, 345, 854

Soderberg, A. M. et al. 2004, ApJ, 606, 994

Soderberg, A. M. et al. 2005, ApJ, 627, 877

Soderberg, A. M. et al. 2006a, Nature, 442, 1014

Soderberg, A. M. et al. 2006b, ApJ, 650, 261

Soderberg, A. M. et al. 2006c, ApJ, submitted (astro-ph/0607511)

Solleman, J. et al. 2006, A\&A, 454, 503

Spergel, D. N. et al. 2006, ApJ, submitted (astro-ph/0603449)

Spitkovsky, A. 2005, AIP Conf. Proc. 801, 345

Spruit, H. C., Daigne, F. \& Drenkhahn, G. 2001, A\&A, 369, 694

Staff, J., Ouyed, R. \& Bagchi, M. A. 2006, ApJ, submitted (astro-ph/0608470)

Stanek, K. Z. et al. 2003, ApJ, 591, L17

Stanek, K. Z. et al. 2006, AcA, in press (astro-ph/0604113)

Stanek, K. Z. et al. 2007, ApJ, 654, L21

Still, M. et al. 2005, ApJ, 635, 1187

Su, M., Li, H., Fan, Z. \& Liu, B. 2006, ApJ, submitted (astro-ph/0611155)

Tagagi, R. \& Kobayashi, S. 2005, ApJ, 622, L25 
Tagliferri, G. et al. 2005, Nature, 436, 985

Tang, S.-M. \& Zhang, S. N. 2006, A\&A, 456, 141

Tanvir, N. R., Chapman, R., Levan, A. J. \& Priddey, R. S. 2005, Nature, 438, 7070

Terasawa, T. et al. 2005, Nature, 434, 1110

Thorne, K. S. 1987, in 300 Years of Gravitation, ed. S. W. Hawking and W. Israel (Cambridge: Cambridge Univ. Press), p330

Thompson, C. 1994, MNRAS, 272, 480

Thompson, C. 2006, ApJ, 651, 333

Thompson, C. \& Duncan, R. C. 1993, ApJ, 408, 194

Thompson, C., Mészáros, P. \& Rees, M. J. 2006, ApJ, submitted (astro-ph/0608282)

Toma, K., R. Yamazaki \& T. Nakamura, 2005, ApJ, 635, 481

Toma, K. et al. 2006, ApJ, 640, L139

Toma, K., Ioka, K., Sakamoto, T. \& Nakamura, T. 2007, ApJ, in press (astro-ph/0610867)

Tominaga, N. et al. 2004, ApJ, 612, L105

Totani, T. 1997, ApJ, 486, L71

Totani, T. 1998, ApJ, 509, L81

Totani, T. et al. 2006, PASJ, 58, 485

Tournefier, E. et al. 2005, EdP-Sciences, Conf. Ser. 539

Troja, E. et al. 2006, GCN Circ \#5055

Usov, V. V. 1992, Nature, 357, 472

Van Elewyck, V. et al. 2006, preprint, (astro-ph/0612731)

van Paradijs et al. 1997, Nature, 386, 686

van Paradijs, C. Kouveliotou, \& Wijers, R. A. M. J. 2000, ARA\&A, 38, 379

van Putten, M. 2001, ApJ, Phys. Rep. 345, 1

Vaughan, S. et al. 2006, ApJ, 638, 920

Vestrand, W. T. et al. 2005, Nature, 435, 178

Vestrand, W. T. et al. 2006, Nature, 442, 7099

Vietri, D. 1995, ApJ, 453, 883

Vietri, D., de Marco, D. \& Guetta, D. 2003, ApJ, 592, 378

Villasenor, J. S. et al. 2005, Nature, 437, 855

Vlahakis, N., Peng, F. \& Königl, A. 2003, ApJ, 594, L23

Wang, F. Y. \& Dai, Z. G. 2006, MNRAS, 368, 371

Wang, X. Y., Cheng, K. S., Dai, Z. G. \& Lu, T. 2004, ApJ, 604, 306

Wang, X. Y., Dai, Z. G. \& Lu, T. 2000, MNRAS, 319, 1159

Wang, X. Y., Dai, Z. G. \& Lu, T. 2001a. ApJ, 546, L33

Wang, X. Y., Dai, Z. G. \& Lu, T. 2001b, ApJ, 556, 1010

Wang, X. Y., Li, Z., Waxman, E. \& Mészáros, P. 2006a, preprint, astro-ph/0608033

Wang, X.-Y., Li, Z. \& Mészáros, P. 2006b, ApJ, 641, L89

Wang, X.-Y. \& Mészáros, P. 2006, ApJ, 643, L95

Wang, X. Y., Wu, X. F., Fan, Y. Z., Dai, Z. G. \& Zhang, B. 2005, ApJ, 623, L29

Watson, D. et al. 2006, ApJ, 637, L69

Waxman, E. 1995, PRL, 75, 386

Waxman, E. 2004a, ApJ, 602, 886

Waxman, E. 2004b, ApJ, 606, 988

Waxman, E. \& Bahcall, J. 1997, PRL, 78, 2292 
Waxman, E. \& Bahcall, J. 1999, PRD, 59, 023002

Waxman, E. \& Bahcall, J. 2000, ApJ, 541, 707

Wei, D. M. 2003, A\&A, 402, L9

Wei, D. M. 2007, MNRAS, 374, 525

Wei, D. M. \& Gao, W. H. 2003, MNRAS, 345, 743

Wei, D. M. \& Lu, T. 1998, ApJ, 505, 252

Wei, D. M. \& Lu, T. 2000a, A\&A, 360, L13

Wei, D. M. \& Lu, T. 2000b, ApJ, 541, 203

Wei, D. M. \& Lu, T. 2002a, A\&A, 381, 731

Wei, D. M. \& Lu, T. 2002b, MNRAS, 332, 994

Wei, D. M., Yan, T. \& Fan, Y. Z. 2006, ApJ, 636, L69

Wei, D. M. \& Jin, Z. P. 2003, A\&A, 400, 415

Wijers, R. A. M. J. \& Galama, T. J. 1999, ApJ, 523, 177

Wijers, R. A. M. J., Rees, M. J. \& Mészáros, P. 1997, MNRAS, 288, L51

Willingale, R. et al. 2006, ApJ, submitted (astro-ph/0612031)

Woods, E. \& Loeb, A. 1995, ApJ, 453, 583

Woosley, S. E. 1993, ApJ, 405, 273

Woosley, S. E. \& Bloom, J. S. 2006, ARA\&A, 44, 507

Woosley, S. E., Eastman, R. G. \& Schmidt, B. P. 1999, ApJ, 516, 788

Wu, X. F., Dai, Z. G., Huang, Y. F. \& Lu, T. 2003, MNRAS, 342, 1131

Wu, X. F., Dai, Z. G. \& Liang, E. W. 2004, ApJ, 615, 359

Wu, X. F., Dai, Z. G., Huang, Y. F. \& Lu, T. 2005b, ApJ, 619, 968

Wu, X. F., Dai, Z. G., Wang, X. Y., Huang, Y. F., Feng, L. L. \& Lu, T. 2005a, preprint (astro$\mathrm{ph} / 0512555)$

$\mathrm{Xu}, \mathrm{D} .2005$, preprint (astro-ph/0504052)

Xu, D., Dai, Z. G. \& Liang, E. W. 2005a, ApJ, 633, 603

Xu, L., Wu, X. F. \& Dai, Z. G. 2005b, ApJ, 634, 1155

Yamazaki, R., Ioka, K. \& Nakamura, T. 2002, ApJ, 571, L31

Yamazaki, R., Ioka, K. \& Nakamura, T. 2004a, ApJ, 606, L33

Yamazaki, R., Ioka, K. \& Nakamura, T. 2004b, ApJ, 607, L103

Yamazaki, R., Toma, R., Ioka, K. \& Nakamura, T. 2006, MNRAS, 369, 311

Yi, T., Liang, E., Qin, Y. \& Lu, R. 2006, MNRAS, 367, 1751

Yonetoku, D. et al. 2004, ApJ, 609, 935

Yost, S. et al. 2003, ApJ, 597, 459

Yu, W. \& Dai, Z. G. 2006, MNRAS, submitted

Zeh, A., Klose, S. \& Hartmann, D. H. 2003, ApJ, 609, 952

Zhang, B. 2005, AIP Conf. Proc. V801, 106 (astro-ph/0509571)

Zhang, B. 2006, Nature, 444, 1010

Zhang, B. 2007, Adv. Spa. Res., in press (astro-ph/0611774)

Zhang, B., Dai, X., Lloyd-Ronning, N. M. \& Mészáros, P. 2004a, ApJ, 601, L119

Zhang, B., Fan, Y. Z., Dyks, J. et al. 2006, ApJ, 642, 354

Zhang, B., Liang, E. W., Page, K. et al. 2007b, ApJ, in press (astro-ph/0607177)

Zhang, B. \& Kobayashi, S. 2005, ApJ, 628, 315

Zhang, B., Kobayashi \& Mészáros, P. 2003a, ApJ, 595, 950

Zhang, B. \& Mészáros, P. 2001a, ApJ, 552, L35 
Zhang, B. \& Mészáros, P. 2001b, ApJ, 559, 110

Zhang, B. \& Mészáros, P. 2002a, ApJ, 566, 712

Zhang, B. \& Mészáros, P. 2002b, ApJ, 571, 876

Zhang, B. \& Mészáros, P. 2002c, ApJ, 581, 1236

Zhang, B. \& Mészáros, P. 2004, IJMPA, 19, 2385

Zhang, B., Zhang, B.-B., Liang, E. W., et al. 2007a, ApJ, 655, L25

Zhang, B.-B., Liang, E.-W. \& Zhang, B. 2007c, ApJ, submitted (astro-ph/0612246)

Zhang, W., Woosley, S. E. \& MacFadyen, A. I. 2003b, ApJ, 586, 356

Zhang, W., Woosley, S. E. \& Heger, A. 2004b, ApJ, 608, 365

Zhao, X. \& Bai, J. M. 2006, ApJ, 653, L1

Zheng, Z., Lu, Y. \& Zhao, Y.-H. 2006, ApJ, 646, L25

Zheng, Z. \& Ramirez-Ruiz, E. 2006, preprint, (astro-ph/0601622)

Zou, Y. C., Dai, Z. G. \& Xu, D. 2006, ApJ, 646, 1098

Zou, Y. C., Wu, X. F. \& Dai, Z. G. 2005, MNRAS,363, 93

This paper was prepared with the ChJAA LATEX macro v1.0. 